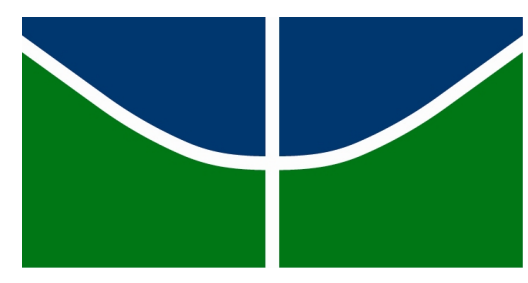

\author{
Universidade de Brasília \\ Instituto de Ciências Exatas \\ Departamento de Matemática
}

\title{
Identidades de Lie da álgebra de Poisson simétrica truncada
}

$$
\text { por }
$$

\section{Ilana Zuila Monteiro Alves}

Orientador: Prof. Dr. Victor Petrogradskiy 
Universidade de Brasília

Instituto de Ciências Exatas

Departamento de Matemática

\section{Identidades de Lie da álgebra de Poisson simétrica truncada}

por

\section{Ilana Zuila Monteiro Alves}

Tese apresentada ao Corpo Docente do Programa de Pós-Graduação em Matemática-UnB, como requisito parcial para obtenção do grau de

\section{DOUTORA EM MATEMÁTICA}

Brasília, 07 de dezembro de 2016.

Comissão Examinadora:

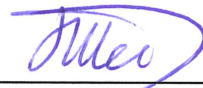

Prof. Dr. Victor Petrogradskiy - Orientador (MAT-UnB)

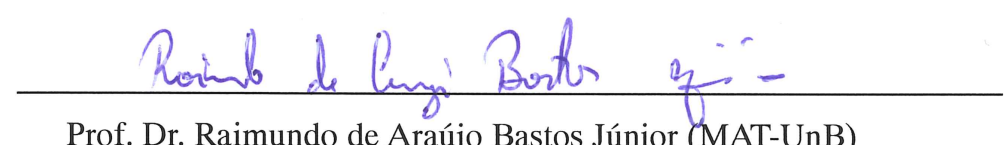

Prof. Dr. Raimundo de Araújo Bastos Júnior (MAT-UnB)

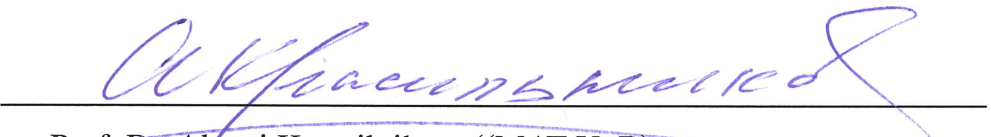

Prof. Dr. Ayexei Krassilnikov ((MAT-UnB)

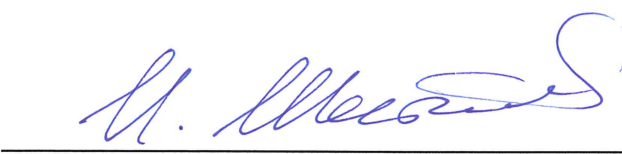

Prof. Dr. Ivan Chestakov (IME/USP)

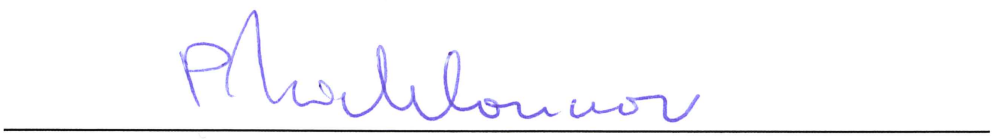

Prof. Dr. Plamen Emilov Kochloukov (UNICAMP)

*A autora foi bolsista CAPES e CNPq durante a elaboração deste trabalho. 
À minha mãe, Francisca

a melhor, mulher batalhadora. 
“... eu pensei que você tivesse me abandonado..."

"... mas você estava me carregando..."

“.. duas pegadas, suas pegadas na areia..."

Footprints, Sia 


\section{Agradecimentos}

A Deus, que me deu a vida, que nos momentos difíceis não me deixou. Sem Ele eu jamais conseguiria essa conquista.

À minha família, pelo amor e compreensão. À minha mãe Francisca, por me amar tanto e me compreender. Aos meus irmãos Aline e Jorge Wilson, pelo amor e carinho de sempre. Ao meu cunhado, Paulo Miguel, por me apoiar e acreditar em mim. Aos meus sobrinhos, Luís Miguel e Vinícius, que são meu descanso e minha alegria.

Aos amigos que fiz em Brasília, que tornaram minha vida nesta cidade mais agradável. Em especial ao Otto, Ali, Somayeh, Bruno, Valter, Claud, Marcos e Keidna. Agradeço à Dona Sandra, por me acolher como uma filha, à Paloma, à Ana Carolina, à Caroline e ao Alessandro, pelo apoio que me deram e pela valiosa amizade.

Agradeço especialmente ao José Carlos e à Saieny, amigos que considero como irmãos, por conseguirem me aturar, por sempre acreditarem em mim, me motivando, me socorrendo e me abrigando quando precisei. Me tornei uma pessoa melhor nestes últimos anos graças à valiosa amizade que temos.

Aos meus amigos de Humaitá, que nesse último ano me apoiaram e me ajudaram significativamente para alcançar essa conquista. Em especial ao Nildo, à Neri, à Cris, ao Cleiton, ao Péricles, ao Yan, ao Állison, ao Adalcir, ao Jonas, à Lucélia, à Paula e ao Euclinger. Aos meus amigos de graduação em Manaus, Ivana, Adrian, Eliane e Ruth que estiveram torcendo por mim esse tempo todo.

Aos professores, membros da comissão avaliadora, Raimundo Bastos, Alexei Krassilnikov, Ivan Shestakov e Plamen Kochloukov, que aceitaram o convite de examinar o texto, por suas sugestões e por terem contribuído no enriquecimento deste trabalho. 
Ao meu orientador, professor Victor Petrogradskiy, por ter me aceitado como sua aluna. Agradeço pela sua paciência, por ter me entregado as chaves dos resultados deste trabalho. Graças aos seus conselhos e dedicação, obtive essa conquista.

À CAPES e ao CNPq pelo apoio financeiro. 


\section{Resumo}

Seja $L$ uma álgebra de Lie sobre um corpo de característica $p>0$. Estudamos a estrutura de álgebra de Lie da álgebra de Poisson simétrica truncada $\mathbf{s}(L)$. Isto é, determinamos as condições necessárias e suficientes para $L$ com respeito as quais $\mathbf{s}(L)$ é Lie nilpotente, Lie nilpotente forte, solúvel e solúvel forte, onde precisamos $p>2$ para estudar a solubilidade. Com respeito as condições estabelecidas, especificamos a classe de Lie nilpotência forte de $\mathbf{s}(L)$. Além disso, provamos que a classe de Lie nilpotência coincide com a classe de Lie nilpotência forte no caso $p>3$.

Em nossa abordagem, usamos uma teoria bem estabelecida de delta-conjuntos para álgebras de Lie e teoria de relações idênticas para álgebras de Poisson. Também estudamos filtrações em álgebras de Poisson e provamos resultados sobre os produtos dos termos das séries centrais inferiores para álgebras de Poisson.

Shestakov provou que a álgebra simétrica $S(L)$ de uma álgebra de Lie arbitrária $L$, satisfaz a identidade de Poisson $\{x,\{y, z\}\} \equiv 0$ se, e somente se, $L$ é abeliana. Estendemos este resultado para Lie nilpotência e Lie solubilidade de $S(L)$.

Palavras-chave: Álgebras simétricas, álgebras de Poisson, Lie nilpotência, Lie solubilidade, álgebras de Lie, PI-álgebras, identidades polinomiais. 


\section{Abstract}

Let $L$ be a Lie algebra over a field of characteristic $p>0$. We study the Lie algebra structure of the truncated symmetric Poisson algebra $\mathbf{s}(L)$. Namely, we determine the necessary and sufficient conditions for $L$ under which $\mathbf{s}(L)$ is Lie nilpotent, Lie strongly nilpotent, solvable and strongly solvable, where we need $p>2$ to study the solvability. Under established conditions, we specify the strong Lie nilpotency class of $\mathbf{s}(L)$. Moreover, we prove that the Lie nilpotency class coincides with the strong Lie nilpotency class in case $p>3$.

In our approach, we use a well-established theory of delta-sets for Lie algebras and theory of identical relations of Poisson algebras. Also, we study filtrations in Poisson algebras and prove results on products of the terms of the lower central series for Poisson algebras.

Shestakov proved that the symmetric algebra $S(L)$ of an arbitrary Lie algebra $L$ satisfies the Poisson identity $\{x,\{y, z\}\} \equiv 0$ if, and only if, $L$ is abelian. We extend this result for Lie nilpotency and Lie solvability of $S(L)$.

Keywords: Symmetric algebras, Poisson algebras, Lie nilpotence, Lie solvability, Lie algebras, PI-algebras, polynomials identities. 


\section{Sumário}

Introdução

1 Preliminares $\quad 5$

1.1 Álgebras de Lie . . . . . . . . . . . . . . . . . . 5

1.2 Noções de álgebras livres . . . . . . . . . . . . . . . . . . . . . . 14

1.3 Álgebra envelopante universal . . . . . . . . . . . . . . . . . . . 22

1.4 Grupos . . . . . . . . . . . . . . . . . . . . . . 41

1.5 Álgebras de Poisson. Exemplos . . . . . . . . . . . . . . . 45

1.6 Álgebra de Poisson livre e identidades de Poisson . . . . . . . . . . . . . . 48

2 Identidades dos anéis de grupos e álgebras envelopantes 52

2.1 Identidades dos anéis de grupos . . . . . . . . . . . . . . 52

2.2 Identidades das álgebras envelopantes universais . . . . . . . . . . . . 54

2.3 Identidades das álgebras envelopantes restritas . . . . . . . . . . . . 55

2.4 Identidades dos produtos smash . . . . . . . . . . . . . 57

2.5 Identidades das álgebras de Poisson simétricas . . . . . . . . . . . . . . 59 
3.1 Duas noções de Lie nilpotência de álgebras de Poisson . . . . . . . . . . . . 61

3.2 Duas noções de Lie solubilidade de álgebras de Poisson . . . . . . . . . . . 62

3.3 Lie nilpotência de álgebras de Poisson simétricas truncadas . . . . . . . . . 64

3.4 Solubilidade de álgebras de Poisson simétricas truncadas . . . . . . . . . . 65

3.4.1 A solubilidade no caso $p=2 \ldots \ldots$. . . . . . . . . 66

4 Lie nilpotência. Demonstrações $\quad 67$

4.1 Delta-conjuntos e identidades de Poisson . . . . . . . . . . . . . . 67

4.2 Filtrações em álgebras de Lie e álgebras simétricas truncadas . . . . . . . . 70

4.3 Lie nilpotência forte da álgebra simétrica truncada . . . . . . . . . . . . 74

4.4 Lie nilpotência da álgebra simétrica truncada $\ldots \ldots$. . . . . . . . . 77

4.5 Produtos de comutadores em álgebras de Poisson . . . . . . . . . . . . . . 79

4.6 A classe de Lie nilpotência . . . . . . . . . . . . . . . . . . 85

5 Lie solubilidade. Demonstrações $\quad 88$

5.1 Lie solubilidade da álgebra simétrica truncada . . . . . . . . . . . . . . . 88

5.2 Solubilidade da álgebra simétrica truncada no caso $p=2 \ldots \ldots$. . . . 98

5.3 Identidades na álgebra de Poisson simétrica . . . . . . . . . . . . . . . . 102

Referências Bibliográficas 


\section{Introdução}

A teoria de PI-álgebras associativas, isto é, de álgebras que satisfazem identidades polinomiais não triviais, é uma área clássica da álgebra moderna. Isto é um importante instrumento para estudar estruturas e propriedades de álgebras associativas. Agora, existe também uma teoria estabelecida de identidades em álgebras de Lie [1]. Ela tem várias aplicações na teoria de grupos tais como a solução do Problema Restrito de Burnside (para maiores detalhes veja $[58,59])$. Também, relações idênticas foram aplicadas ao estudo de outras estruturas algébricas.

O primeiro ponto de partida para nossa pesquisa é o resultado de Passman sobre a existência de identidades em anéis de grupos [34] (Teorema 2.1.1, na página 52 deste texto). Este artigo causou uma intensa pesquisa sobre diferentes tipos de identidades em anéis de grupos, tais como Lie nilpotência, solubilidade, identidades não matriciais, classes de Lie nilpotência, comprimentos de solubilidade, etc. Existem pelo menos 50 trabalhos publicados nesta área.

Latyshev [26] e Bahturin [2] iniciaram o estudo de identidades em álgebras envelopantes universais de álgebras de Lie. O segundo ponto de partida para nossa pesquisa é o resultado de Passman [36] e Petrogradsky [37] sobre a existência de identidades nas álgebras envelopantes restritas (Teorema 2.3.1, na página 55 deste texto). Existem vários artigos nesta área onde são estudados diferentes tipos de identidades, tais como Lie nilpotência, solubilidade, identidades não matriciais, classes de Lie nilpotência, comprimentos de solubilidade, etc. Assim, Riley e Shalev determinaram condições necessárias e suficientes para álgebras de Lie restritas nas quais a álgebra envelopante restrita é Lie nilpotente ou solúvel [42]. Também a pesquisa foi estendida a novos objetos, tais como superálgebras de Lie, superálgebras de Lie coloridas e produtos smash. Estes problemas 
foram estudados em numerosos trabalhos por Bahturin, Kochetov, Petrogradsky, Riley, Shalev, Siciliano, Spinelly, Usefi, et. al.

Álgebras de Poisson foram definidas primeiro nos trabalhos de Berezin [7] e Vergne [55]. Álgebras de Poisson foram usados para estudar álgebras envelopantes universais de álgebras de Lie, para álgebras de Lie de dimensão finita em característica zero. Álgebras de Poisson surgem naturalmente em diferentes áreas da álgebra, topologia e física. Aplicando álgebras de Poisson, Shestakov e Umirbaev conseguiram resolver um problema de longa data: eles provaram que o automorfismo de Nagata do anel polinomial em três variáveis $\mathbb{C}[x, y, z]$ é "wild", (para mais detalhes veja [45]). Propriedades algébricas relacionadas de álgebras de Poisson livres foram estudadas por Makar-Limanov, Shestakov e Umirbaev [27], [28].

As álgebras de Poisson livres foram definidas por Shestakov [44]. A teoria básica de identidades para álgebras de Poisson foi desenvolvida por Farkas [13, 14]. Vemos mais desenvolvimentos sobre a teoria de identidades para álgebras de Poisson e aplicações às então chamadas crescimento de codimensões em característica zero por Mishchenko, Petrogradsky e Regev [29].

Continuamos a estudar identidades de álgebras de Poisson simétricas (truncadas) de (super)álgebras de Lie iniciado por Kostant [22], Shestakov[44], e Farkas [14]. O terceiro ponto de partida para nossa pesquisa é o resultado de Giambruno e Petrogradsky [15] sobre a existência de identidades de Poisson multilineares não triviais nas álgebras simétricas truncadas das álgebras de Lie restritas (Teorema 2.5.5, na página 60 deste texto).

A presente tese é dedicada à questão de quando álgebras simétricas truncadas das álgebras de Lie sobre um corpo de característica positiva satisfazem as identidades de Lie nilpotência, Lie nilpotência forte, solubilidade e solubilidade forte. Também determinamos as respectivas classes de nilpotência.

Nosso trabalho está estruturadamente dividido em cinco capítulos. No Capítulo 1, apresentamos conceitos básicos, mas que são fundamentais para o desenvolvimentos dos demais capítulos. Incluímos elementos da teoria de álgebras de Lie como definições e noções de álgebras de Lie, noções de álgebras de Lie nilpotente e solúvel, bem como resultados que relacionam as subálgebras e ideais de Lie a estes dois conceitos. Também apresentamos algumas noções de álgebras associativas e de Lie livres, onde para esta última fazemos a construção de uma base (como $K$-espaço vetorial) para seus elementos, 
a base de Hall e introduzimos daí, a definição de PI-álgebras. Destacamos a existência de uma álgebra envelopante universal para uma álgebra de Lie e enunciamos o Teorema de Poincaré-Birkhoff-Witt. Em uma das seções, recordamos alguns objetos da teoria de grupos e definimos um anel de grupo. Por fim, nós apresentamos a definição de álgebras de Poisson, damos alguns exemplos típicos e também introduzimos as álgebras de Poisson livre e identidades de Poisson.

No Capítulo 2, apresentamos um compêndio de resultados descobertos por vários matemáticos com respeito a identidades satisfeitas pelas várias estruturas apresentadas no Capítulo 1. Estes resultados foram valiosas ferramentas para nosso trabalho, portanto nós apresentamos cada um dos seus enuncidados. Neste capítulo, estão resultados das identidades de anéis de grupos, álgebras envelopantes, dos produtos smash de anéis de grupos e álgebras envelopantes, e das identidades das álgebras de Poisson.

No Capítulo 3, apresentamos nossos resultados principais sobre Lie nilpotências e solubilidades da álgebra simétrica truncada de uma álgebra de Lie sobre um corpo de característica $p>0$. Introduzimos duas noções de Lie nilpotência que são dadas por meio das séries centrais inferiores e potências de Lie superiores de álgebras de Poisson e duas noções de Lie solubilidade que são dadas por meio das séries derivadas de álgebras de Poisson. O ponto de partida para o Capítulo 4, é a demonstração do nosso primeiro resultado principal em que estabelecemos condições necessárias e suficientes para $L$ as quais nos permitem definir identidades de Lie nilpotência e Lie nilpotência forte satisfeitas por $\mathbf{s}(L)$. Neste capítulo nós apresentamos os delta-conjuntos e suas propriedades que são conceitos fundamentais para as nossas provas. Definimos as filtrações em álgebras de Lie e álgebras simétricas truncadas e apresentamos um resultado chave que relaciona estas duas filtrações. Desta relação é possível especificar a classe de Lie nilpotência forte da álgebra simétrica truncada. Também apresentamos alguns resultados que nos permitem concluir que a classe de Lie nilpotência coincide com a classe de Lie nilpotência forte. Nós seguimos uma abordagem próxima a que foi feita por Krasilnikov em [23].

No Capítulo 5, nós demonstramos o nosso segundo resultado principal, que é sobre a Lie solubidade da álgebra simétrica truncada de uma álgebra de Lie sobre um corpo de característica $p \geq 3$. Também para estes resultados, apresentamos condições necessárias e suficientes que a álgebra de Lie $L$ precisa ter para definirmos as identidades de Lie solúvel e Lie solúvel forte satisfeitas por $\mathbf{s}(L)$. Neste capítulo, usamos o conceito de deltaconjuntos definido no Capítulo 4. Construímos dois exemplos de álgebras simétricas truncadas que são solúveis mas que não são solúveis fortes sobre corpos de característica 
$p=2$. Finalizamos nossos trabalhos apresentando uma generalização dos resultados de Shestakov [44] das identidades da álgebra de Poisson simétrica sobre um corpo arbitrário. 


\section{Capítulo 1}

\section{Preliminares}

Neste capítulo apresentamos definições e resultados que serão importantes para o desenvolvimento do nosso trabalho. Primeiro, falaremos de alguns objetos da teoria de álgebras de Lie, tais como álgebras de Lie, subálgebras, ideais, morfismos, álgebras de Lie nilpotentes e solúveis. Depois, vamos introduzir as noções de álgebras livres, como álgebras associativas livres e álgebras de Lie livres. A partir dos elementos destas estruturas, definiremos as álgebras que satisfazem identidades polinomiais não triviais e que são assim chamadas PI-álgebras. Vamos recordar os conceitos de álgebras envelopantes universais de uma álgebra de Lie, de grupos e de anéis de grupos. Introduziremos a definição de álgebras de Poisson, dando quatro exemplos delas e mencionamos as álgebras de Poisson livres e identidades de Poisson.

\section{1 Álgebras de Lie}

Vamos recordar a definição de álgebra não associativa sobre um corpo $K$.

Definição 1.1.1 Uma álgebra não associativa $A$ sobre um corpo $K$ é um espaço vetorial no qual é definido uma operação bilinear $: A \times A \rightarrow A$, chamada produto tal que, para quaisquer $a, b, c \in A$ e qualquer $\alpha \in K$, satisfaz os seguintes axiomas:

1. $(a+b) \cdot c=a \cdot c+b \cdot c ;$ 
2. $a \cdot(b+c)=a \cdot b+a \cdot c$;

3. $\alpha(a \cdot b)=(\alpha a) \cdot b=a \cdot(\alpha b)$.

Se a álgebra $A$ satisfaz

$$
a \cdot(b \cdot c)=(a \cdot b) \cdot c,
$$

para todos $a, b, c \in A$, então é chamada álgebra associativa.

Dada a definição acima, denotaremos a partir de agora, o produto $a \cdot b$ apenas por $a b$.

Seja $A$ uma álgebra associativa. Denotaremos por $A^{(-)}$(o mesmo espaço) munido com um produto dado por

$$
[x, y]:=x y-y x, \quad x, y \in A \text {. }
$$

Este produto em $A^{(-)}$satisfaz, para todos $x, y, z \in A$, as seguintes relações:

1. $[x, y]=-[y, x]$ (anticomutatividade ou antissimétrica);

2. $[[x, y], z]+[[y, z], x]+[[z, x], y] \equiv 0$ (Identidade de Jacobi).

O espaço $A^{(-)}$, com o produto (1.1), é chamado álgebra de Lie. A seguir, iremos introduzir a definição formal de álgebras de Lie.

Definição 1.1.2 Seja L um espaço vetorial sobre um corpo $K$. Dizemos que L é uma álgebra de Lie ou $K$-álgebra de Lie se $L$ é munido com um produto chamado de colchete ou comutador de Lie

$$
[\cdot, \cdot]: L \times L \rightarrow L
$$

que satisfaz as seguintes propriedades de Lie:

1. é bilinear;

2. é anticomutativo, isto é, $[x, x]=0$ para todo $x \in L$;

3. satisfaz a identidade de Jacobi, isto é, para todo $x, y, z \in L$

$$
[[x, y], z]+[[y, z], x]+[[z, x], y]=0
$$


Dessa forma, pela definição acima, o espaço $A^{(-)}$é chamado de álgebra de Lie associada a álgebra associativa $A$.

Antes de prosseguirmos com nossas definições, vamos adotar o produto normado à esquerda. Isto é, se $A$ é uma álgebra não necessariamente associativa, então o produto normado à esquerda dos elementos $x_{1}, x_{2}, \ldots, x_{n} \in A$ é definido por indução como

$$
\left[x_{1}, x_{2}, \ldots, x_{n}\right]=\left[\ldots\left[\left[x_{1}, x_{2}\right], x_{3}\right], \ldots, x_{n}\right], \quad \text { se } n>1 \text {. }
$$

Se $n=1$, colocamos apenas $x_{1}$. Além disso, escrevemos, para simplificar,

$$
[y, \underbrace{x, x, \ldots, x}_{m}]=\left[y,_{m} x\right] .
$$

Note que se $L$ é uma álgebra de Lie e $x, y \in L$ são quaisquer, segue da propriedade antissimétrica de $[\cdot, \cdot]$ que

$$
0=[x+y, x+y]=[x, x]+[x, y]+[y, x]+[y, y]=[x, y]+[y, x]
$$

de modo que

$$
[x, y]=-[y, x]
$$

vale para qualquer álgebra de Lie. Reciprocamente, se esta condição vale, então $2[x, x]=$ 0 , de modo que, se a característica de $K$ não é dois, então $[x, x]=0$. Portanto, para álgebras de Lie sobre corpos de característica diferente de dois, a condição (1.2) pode ser usada no lugar da propriedade 2 da Definição 1.1.2. Além disso, dizemos que dois elementos $x, y \in L$ são comutáveis se $[x, y]=0$.

Definição 1.1.3 Uma álgebra de Lie L é dita abeliana se quaisquer dois elementos de $L$ são comutáveis.

Agora daremos algumas noções de morfismos, quociente e produto. Essas noções fazem sentido e funcionam da mesma forma para uma grande variedade de estruturas algébricas e alguns deles serão catalogadas, a seguir, para as álgebras de Lie.

Definição 1.1.4 Uma transformação linear $\varphi: L_{1} \rightarrow L_{2}$, (com $L_{1}$ e $L_{2}$ álgebras de Lie) é um: 
1. homomorfismo de álgebras de Lie se $\varphi\left([x, y]_{L_{1}}\right)=[\varphi(x), \varphi(y)]_{L_{2}}$, para quaiquer $x, y \in L_{1}$;

2. isomorfismo de álgebras de Lie se for um homomorfismo de álgebras de Lie invertivel;

3. automorfismo de $L_{1}$ se é um isomorfismo de álgebras de Lie e $L_{1}=L_{2}$.

Duas álgebras de Lie $L_{1}$ e $L_{2}$ são ditas isomorfas se existe um isomorfismo $\varphi: L_{1} \rightarrow L_{2}$.

Seja $L$ uma álgebra de Lie sobre um corpo $K$ e sejam $H_{1}$ e $H_{2} K$-subespaços vetoriais de $L$. Então definimos

$$
\left[H_{1}, H_{2}\right]:=\left\langle\left[h_{1}, h_{2}\right] \mid h_{1} \in H_{1}, h_{2} \in H_{2}\right\rangle_{K}
$$

o subespaço de $L$ gerado pelo comutador dos elementos de $H_{1}$ e $H_{2}$. Note que $\left[H_{1}, H_{2}\right]=$ $\left[H_{2}, H_{1}\right]$.

Definição 1.1.5 Seja L uma álgebra de Lie sobre um corpo $K$ e seja $H$ subespaço de $L$. Então

1. $H$ é chamado subálgebra de Lie ou simplesmente de subálgebra de $L$ se $[H, H] \subseteq H$;

2. $H$ é chamado ideal de Lie ou apenas de ideal de $L$ se $[H, L] \subseteq H$.

Em particular, o subespaço $[L, L]=\left\langle\left[l_{1}, l_{2}\right] \mid l_{1}, l_{2} \in L\right\rangle_{K}$ é um ideal de $L$ chamado ideal comutador, ou derivado de $L$ e é denotado por $L^{\prime}$ ou ainda por $L^{2}$.

Seja $A$ uma $K$-álgebra. Um endomorfismo $\delta$ do espaço vetorial de $A$ é chamado uma derivação se para quaisquer $x, y \in A$, tem-se

$$
\delta(x, y)=\delta(x) y+x \delta(y)
$$

Exemplo 1.1.6 Seja L uma álgebra de Lie e seja $D(L)$ o espaço vetorial de todas as derivações de $L$, isto é, operadores lineares do espaço vetorial L que satisfazem

$$
\delta([x, y])=[\delta(x), y]+[x, \delta(y)], \quad \delta \in D(L), \quad x, y \in L
$$


$D(L)$ é uma álgebra de Lie com produto definido como

$$
[\delta, \theta](a)=\delta(\theta(a))-\theta(\delta(a)), \quad \delta, \theta \in D(L)
$$

Agora, seja $x \in L$, a aplicação ad $x: L \rightarrow L$, dada por ad $x(y)=[x, y], y \in L$, é uma derivação. Tal derivação é chamada interna. O conjunto ad $L=\{\operatorname{ad} x \mid x \in L\}$ das derivações internas de $L$ é um ideal de Lie de $D(L)$.

Exemplo 1.1.7 Seja L uma álgebra de Lie. O centro de L é o subconjunto

$$
Z(L)=\{x \in L \mid[x, y]=0, \forall y \in L\}
$$

$O$ centro de uma álgebra de Lie $L$ é um ideal. De fato, como $[0, y]=0$, para todo $y \in L, \log o \in Z(L)$. Se $y \in L$ e $x \in Z(L)$, então $[x, y]=0 \in Z(L)$.

Definição 1.1.8 Seja L uma álgebra de Lie sobre um corpo $K$ e seja $S$ um subconjunto de L. Definimos:

1. a subálgebra de Lie L gerada por $S$, denotada por Lie $(S)$, como sendo a interseção de todas as subálgebras de $L$ que contém $S$;

2. o ideal de $L$ gerado por $S$ como sendo a interseção de todos os ideais de $L$ que contém $S$.

Se $L=\operatorname{Lie}(S)$, então dizemos que $S$ gera $L$ como álgebra, ou que $S$ é um conjunto gerador de $L$ como álgebra. Uma caracterização de subálgebra e ideal gerado por um subconjunto de uma álgebra de Lie é dado a seguir.

1. A subálgebra de $L$ gerada por $S$ coincide com o subespaço de $L$ gerado pelo conjunto

$$
\left\{\left[s_{i_{1}}, s_{i_{2}}, \ldots, s_{i_{n}}\right] \mid i_{j} \in \mathbb{N}, s_{i} \in S\right\}
$$

2. O ideal de $L$ gerado por $S$ coincide com o subespaço de $L$ gerado por

$$
\left\{\left[s, a_{1}, \ldots, a_{k}\right] \mid s \in S, a_{i} \in L, k \geq 0\right\}
$$


As propriedades de soma e de interseção de ideais e subálgebras estão catalogadas no seguinte resultado.

Proposição 1.1 .9 ([31], pág. 9) Sejam $H_{1}$ e $H_{2}$ subespaços de L sobre o corpo $K e$ defina $H_{1}+H_{2}:=\left\{h_{1}+h_{2} \mid h_{1} \in H_{1}, h_{2} \in H_{2}\right\}$ e $H_{1} \cap H_{2}:=\left\{h \mid h \in H_{1}, h \in H_{2}\right\}$. Então

1. se $H_{1}$ e $H_{2}$ são ideais, então $H_{1}+H_{2}$ e $H_{1} \cap H_{2}$ são ideais;

2. Se $H_{1}$ é subálgebra e $H_{2}$ é ideal, então $H_{1}+H_{2}$ e $H_{1} \cap H_{2}$ são subálgebras;

3. Se $H_{1}$ e $H_{2}$ são subálgebras, então $H_{1} \cap H_{2}$ é uma subálgebra.

Agora, sejam $L_{1}$ e $L_{2}$ álgebras de Lie, com respeito ao núcleo e a imagem de um homomorfismo entre essas álgebras, temos o seguinte teorema.

Teorema 1.1.10 ([31], pág. 10) Seja $\varphi: L_{1} \rightarrow L_{2}$ um homomorfismo de álgebras de Lie. Então

1. o núcleo $\operatorname{ker} \varphi$ é um ideal de $L_{1}$;

2. a imagem $\operatorname{Im} \varphi$ é uma subálgebra de $L_{2}$.

Sejam $L$ uma álgebra de Lie sobre um corpo $K$ e $H$ um ideal de $L$. O espaço quociente

$$
L / H:=\{\bar{x}=x+H \mid x \in L\}
$$

consistindo das classes laterais $\bar{x}=x+H:=\{x+h \mid h \in H\}$ para $x \in L$, é uma álgebra de Lie definindo a soma e o produto de classes por

$$
\begin{gathered}
\bar{x}+\bar{y}:=\overline{x+y} \text { e } \lambda \bar{x}:=\overline{\lambda x} \\
\text { e }[\bar{x}, \bar{y}]:=\overline{[x, y]}
\end{gathered}
$$

para todo $x, y \in L$ e todo $\lambda \in K$. Estas operações estão bem definidas pois $H$ é um ideal, e ele herda diretamente todos os axiomas de $L$.

Existe um homomorfismo sobrejetor $\phi: L \rightarrow L / H$, dado por $x \mapsto \bar{x}=x+H$, de álgebras de Lie chamado de homomorfismo canônico. 
Assim como no caso de álgebras associativas, obtemos resultados análogos para álgebras de Lie dos seguintes teoremas.

Teorema 1.1.11 (Primeiro Teorema dos Isomosfismos, [31], pág. 10) Seja $\varphi: L \rightarrow$ $H$ um homomorfismo de álgebras de Lie sobre um corpo $K \operatorname{com} I=\operatorname{ker} \varphi$. Então,

$$
\begin{aligned}
\psi: L / I & \rightarrow \operatorname{Im} \varphi \\
x+I & \mapsto \varphi(x)
\end{aligned}
$$

é um isomorfismo de álgebras de Lie.

Demonstração. A aplicação $\psi$ está bem definida uma vez que $\bar{x}=\bar{y}$ é equivalente a $x-y \in I=\operatorname{ker} \varphi$ e $\operatorname{assim} \psi(x)=\psi(y)$. Isto também prova que $\psi$ é injetiva, e a sobrejetiva da imagem de $\varphi$ é por construção. A aplicação $\psi$ é claramente $K$-linear e um homomorfismo de álgebras pois $\varphi$ o é.

Teorema 1.1.12 (Segundo Teorema dos Isomorfismos, [31], pág. 10) Sejam L uma álgebra de Lie sobre um corpo $K, I$ um ideal e $H$ uma subálgebra. Então $H \cap I$ é um ideal de $H$ e a aplicação

$$
\begin{aligned}
\psi: \begin{array}{c}
\frac{H}{(H \cap I)} \\
h+(H \cap I)
\end{array} & \mapsto \frac{(H+I)}{I} \\
& \mapsto h+I
\end{aligned}
$$

é um isomorfismo de álgebras de Lie.

Demonstração. O ideal $I$ de $L$ é automaticamente um ideal da subálgebra $H+I$ (veja o item 3. da Proposição 1.1.9). Agora, definindo a aplicação $\bar{\psi}: H \rightarrow(H+I) / I$ por $\bar{\psi}(h):=h+I$, teremos claramente que esta aplicação é um homomorfismo de álgebras de Lie. Sua imagem é $(H+I) / I$ uma vez que cada classe lateral tem um representante em $H$. O núcleo de $\bar{\psi}$ é exatamente $H \cap I$ e segue que este é um ideal em $H$.

\section{Álgebras de Lie nilpotentes e solúveis}

Seja $L$ uma álgebra de Lie sobre um corpo $K$. Definimos, por indução, a série central inferior de $L$ como $\gamma_{1}(L)=L$ e $\gamma_{n}(L)=\left[\gamma_{n-1}(L), L\right]$ para $n \geq 2$. Isto nos dá uma 
sequência decrescente de ideais

$$
L=\gamma_{1}(L) \supseteq L^{2}=[L, L]=\gamma_{2}(L) \supseteq \cdots \supseteq \gamma_{n}(L) \supseteq \cdots
$$

Definição 1.1.13 A álgebra de Lie L é chamada nilpotente, se existe um $n \in \mathbb{N}$ tal que $\gamma_{n}(L)=0$.

Se $s \in \mathbb{N}$ é o menor tal que $\gamma_{s+1}(L)=0$, então dizemos que $L$ é uma álgebra de Lie nilpotente de classe $s$.

Também define-se, por indução, os termos da série derivada de $L$ como $\delta_{0}(L)=L$ e $\delta_{n}(L)=\left[\delta_{n-1}(L), \delta_{n-1}(L)\right]$ para $n \geq 1$. Isto nos dá a sequência decrescente de ideais

$$
L=\delta_{0}(L) \supseteq L^{2}=[L, L]=\delta_{1}(L) \supseteq \cdots \supseteq \delta_{n}(L) \supseteq \cdots
$$

Definição 1.1.14 A álgebra de Lie L é chamada solúvel, se existe um $n \in \mathbb{N}$ tal que $\delta_{n}(L)=0$.

Se $s \in \mathbb{N}$ é o menor tal que $\delta_{s}(L)=0$, então dizemos que $L$ é uma álgebra de Lie solúvel de classe $s$.

O próximo teorema relaciona as definições das séries dadas acima com os ideais e subálgebras de uma álgebra de Lie.

Teorema 1.1.15 ([31], pág. 12) Sejam L uma álgebra de Lie, H uma subálgebra e I um ideal. Então,

1. se L é abeliana, nilpotente ou solúvel, então o mesmo é válido para $H$ e L/I;

2. se I e L/I são solúveis, então L é solúvel também;

3. se $L / Z(L)$ é nilpotente, então L também é;

4. $\left[\gamma_{k}(L), \gamma_{m}(L)\right] \subseteq \gamma_{k+m}(L)$ para todo $k, m \in \mathbb{N}$;

5. $\delta_{n}(L) \subseteq \gamma_{2^{n}}(L)$ para todo $n \in \mathbb{N}$;

6. toda álgebra de Lie nilpotente é solúvel; 
7. se $L$ é nilpotente e $L \neq 0$, então $Z(L) \neq 0$.

Demonstração. Considere a afirmação 1, supondo $L$ uma álgebra de Lie abeliana. Então claramente todos os produtos em $H$ e $L / I$ são zero, assim temos 1 para este caso. Para os casos nilpotentes e solúveis as inclusões

$$
\gamma_{i}(H) \subseteq \gamma_{i}(L) \quad \text { e } \quad \delta_{i}(H) \subseteq \delta_{i}(L)
$$

e as igualdades

$$
\gamma_{i}(L / I)=\left\{x+I \mid x \in \gamma_{i}(L)\right\} \quad \text { e } \quad \delta_{i}(L / I)=\left\{x+I \mid x \in \delta_{i}(L)\right\}
$$

implicam imediatamente em 1.

Para 2, assumimos que ambos $I$ e $L / I$ são solúveis, isto é, existem $m, k \in \mathbb{N}$ tais que $\delta_{m}(L / I)=\{0+I\}$ e $\delta_{k}(I)=0$. Mas isto implica diretamente que $\delta_{m}(L) \subseteq I$ e assim $\delta_{m+k}(L)=0$ o qual mostra que $L$ é solúvel.

Para provar 3, assumimos que $\gamma_{n}(L) \subseteq Z(L)$, então

$$
\gamma_{n+1}(L)=\left[\gamma_{n}(L), L\right] \subseteq[Z(L), L]=0
$$

Agora, nós consideraremos 4. A prova é feita por indução sobre $m$, fixando $k \geq 1$. A afirmação vale para $m=1$ pela definição de $\gamma_{k+1}(L)=\left[\gamma_{k}(L), L\right]$. Agora vamos supor que a afirmação 4 . é válida para $m \leq r$ e para todo $k$. Então pela identidade de Jacobi na forma $[[x, y], z]=-[[y, z], x]-[[z, x], y]$, para todos $x, y, z \in L$ e pelo argumento de indução, segue que

$$
\begin{aligned}
{\left[\gamma_{k}(L), \gamma_{r+1}(L)\right] } & =\left[\gamma_{k}(L),\left[\gamma_{r}(L), L\right]\right] \\
& =\left[\left[\gamma_{r}(L), L\right], \gamma_{k}(L)\right] \\
& \subseteq\left[\left[L, \gamma_{k}(L)\right], \gamma_{r}(L)\right]+\left[\left[\gamma_{k}(L), \gamma_{r}(L)\right], L\right] \\
& \subseteq\left[\gamma_{k+r}(L), L\right]+\left[\gamma_{k+1}(L), L\right] \\
& \subseteq \gamma_{k+r+1}(L)
\end{aligned}
$$


A afirmação 5 segue por indução sobre $n$. Temos que $\delta_{0}(L)=L=\gamma_{1}(L)=\gamma_{2^{0}}(L)$, para o caso $n=1$. Vamos supor que para $n>1$ tenhamos $\delta_{n}(L) \subseteq \gamma_{2^{n}}(L)$, então

$$
\delta_{n+1}(L)=\left[\delta_{n}(L), \delta_{n}(L)\right] \subseteq\left[\gamma_{2^{n}}(L), \gamma_{2^{n}}(L)\right] \subseteq \gamma_{2^{n}+2^{n}}(L)=\gamma_{2^{n+1}}(L)
$$

usando a afirmação 4 .

Para a afirmação 6, suponhamos que $L$ é nilpotente de classe $n$, isto é, existe $n$ tal que $\gamma_{n+1}(L)=0$. Assim, para todo $k>n$, vamos ter $\gamma_{k}(L)=0$. Mas então, $\delta_{n}(L) \subseteq \gamma_{2^{n}}(L)=0$ (uma vez que $2^{n}>n$, para todo $n \geq 0$ ). Desta forma, segue que $L$ é solúvel.

Agora, para a afirmação 7 , note que se $L$ é nilpotente de classe $n$, então $\gamma_{n+1}(L)=$ $\left[\gamma_{n}(L), L\right]=0$, mas $\gamma_{n}(L) \neq 0$. Este último termo da série central inferior é não nulo e está contido no centro de $Z(L)$.

\subsection{Noções de álgebras livres}

Nesta seção iremos assumir todas as definições e resultados com respeito as variedades de álgebras.

\section{Álgebras associativas livres}

Antes de introduzirmos o conceito de álgebras associativas livres, vamos recordar a definição de produto tensorial de espaços vetoriais, em seguida definiremos a estrutura de anéis graduados.

Definição 1.2.1 Sejam $V$ e $W$ espaços vetoriais, sobre o corpo $K$, com bases $\left\{v_{i} \mid i \in I\right\}$ $e\left\{w_{j} \mid j \in J\right\}$, respectivamente. O produto tensorial $V \otimes W=V \otimes_{K} W$ de $V$ e $W$ é o espaço vetorial com base $\left\{v_{i} \otimes w_{j} \mid i \in I, j \in J\right\}$. Definiremos em $V \otimes W$ a seguinte relação

$$
\left(\sum_{i \in I} \alpha_{i} v_{i}\right) \otimes\left(\sum_{j \in J} \beta_{j} w_{j}\right)=\sum_{i \in I} \sum_{j \in J} \alpha_{i} \beta_{j}\left(v_{i} \otimes w_{j}\right), \quad v_{i} \in V, w_{j} \in W, \alpha_{i}, \beta_{j} \in K
$$

Os elementos de $V \otimes W$ satisfazem as seguintes propriedades: 
1. $\left(v_{1}+v_{2}\right) \otimes w=\left(v_{1} \otimes w\right)+\left(v_{2} \otimes w\right) ;$

2. $v \otimes\left(w_{1}+w_{2}\right)=\left(v \otimes w_{1}\right)+\left(v \otimes w_{2}\right) ;$

3. $(\lambda v) \otimes w=\lambda(v \otimes w)$

4. $v \otimes(\lambda w)=\lambda(v \otimes w)$;

para quaisquer $v_{1}, v_{2}, v \in V, w_{1}, w_{2}, w \in W$ e $\lambda \in K$.

Sejam $A$ e $B K$-álgebras e consideremos em $A \otimes B$ o produto bilinear definido por

$$
\begin{aligned}
\cdot(A \otimes B) \times(A \otimes B) & \longrightarrow(A \otimes B) \\
\left(\left(a_{1} \otimes b_{1}\right),\left(a_{2} \otimes b_{2}\right)\right) & \longmapsto\left(a_{1} \otimes b_{1}\right) \cdot\left(a_{2} \otimes b_{2}\right)=a_{1} a_{2} \otimes b_{1} b_{2} .
\end{aligned}
$$

O espaço vetorial, munido com este produto, é uma $K$-álgebra chamada de produto tensorial das álgebras $A$ e $B$.

\section{Caracterização por uma propriedade universal}

O produto tensorial de espaços vetoriais, definido como acima, satisfaz a seguinte propriedade universal: existe uma aplicação bilinear $\otimes: V \times W \rightarrow V \otimes W$ tal que, para qualquer outro espaço vetorial $U$ junto com uma aplicação $\varphi: V \times W \rightarrow U$, existe uma única aplicação linear $\psi: V \otimes W \rightarrow U$ que satisfaz $\varphi=\psi \circ \otimes$, ou seja, que faz o diagrama

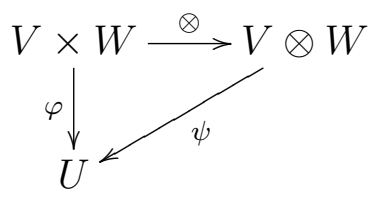

comutar.

Além disso, se $(Z, \widetilde{\otimes})$ é um outro par satisfazendo a mesma propriedade universal, então $Z \cong V \otimes W$.

O produto tensorial também opera em aplicações lineares entre espaços vetoriais. Especificamente, dadas duas aplicações lineares $\varphi: V \rightarrow U$ e $\psi: W \rightarrow U$ entre espaços vetoriais, o produto tensorial das duas aplicações lineares $\varphi$ e $\psi$ é uma aplicação linear 


$$
\varphi \otimes \psi: V \otimes W \rightarrow U
$$

dada por

$$
(\varphi \otimes \psi)(v \otimes w)=\varphi(v) \otimes \psi(w), v \in V, w \in W
$$

Recordemos rapidamente a definição de anel graduado, ideal graduado e álgebra quociente graduada.

Definição 1.2.2 1. Um anel $R$ é chamado um anel graduado se ele é a soma direta de subgrupos aditivos: $R=R_{0} \oplus R_{1} \oplus R_{2} \oplus \cdots$ tais que $R_{n} R_{m} \subseteq R_{n+m}$ para todos $n, m \geq 0$. Os elementos de $R_{n}$ são ditos elementos homogêneos de $R$ de grau $n e$ $R_{n}$ é chamado a componente homogênea de $R$.

2. Um ideal $I$ do anel graduado $R$ é chamado um ideal graduado se $I=\bigoplus_{n=0}^{\infty}\left(I \cap R_{n}\right)$.

3. Um homomorfismo $\varphi: R \rightarrow S$ entre dois anéis graduados é chamado um homomorfismo graduado se $\varphi\left(R_{n}\right)=S_{n}$, para $k=0,1,2, \ldots$

Além disso, se $R$ é um anel graduado e $I$ um ideal graduado de $R$, colocando $I_{n}=I \cap$ $R_{n}$, para todo $n \geq 0$, temos que $R / I$ é naturalmente um anel graduado cujas componentes homogêneas de grau $n$ são isomórficas a $R_{n} / I_{n}$. Isto é demonstrado provando que a aplicação

$$
\begin{array}{ccc}
R=\oplus R_{n} & \rightarrow & \oplus\left(R_{n} / I_{n}\right) \\
\left(\ldots, r_{n}, \ldots\right) & \mapsto \quad\left(\ldots, r_{n} \bmod I_{n}, \ldots\right)
\end{array}
$$

é sobrejetiva com núcleo $I=\oplus I_{n}$ e portanto, define um isomorfismo de anéis graduados.

Agora, seja $X=\left\{x_{i} \mid i \in I\right\}$ um conjunto não vazio de variáveis e seja $V=\left\langle x_{i}\right| i \in$ $I\rangle_{K}$ o espaço vetorial sobre um corpo $K$ com base em $X$.

A álgebra tensorial $A=A(X)$ do espaço vetorial $V$ sobre $K$ é dada por

$$
A(X)=\langle 1\rangle \oplus V \oplus V \otimes V \oplus \cdots \oplus \underbrace{V \otimes \cdots \otimes V}_{=V^{\otimes n}} \oplus+\cdots
$$

com o produto denotado por $*$ e definido por

$$
\left(x_{1} \otimes \cdots \otimes x_{n}\right) *\left(y_{1} \otimes \cdots \otimes y_{m}\right)=x_{1} \otimes \cdots \otimes x_{n} \otimes y_{1} \otimes \cdots \otimes y_{m} .
$$


Estendemos este produto pela lei distributiva. A base de $A(X)$ são todos os monômios nos elementos de $X$. Vamos dizer que dois monômios $x_{i_{1}} \otimes \cdots \otimes x_{i_{n}}$ e $x_{j_{1}} \otimes \cdots \otimes x_{j_{k}}$ em $A$ são iguais se, e somente se, $i_{1}=j_{1}, \ldots, i_{n}=j_{k}$ e $n=k$. Com respeito a esta multiplicação temos $V^{\otimes n} * V^{\otimes m} \subseteq V^{\otimes n+m}$.

Este produto é associativo e a álgebra $A=A(X)$ pode ser decomposta naturalmente como

$$
A=\bigoplus_{n=0}^{\infty} A_{n}, \quad \text { onde } \quad A_{n}=V^{\otimes n}
$$

onde os elementos de $A_{n}$ são chamados monômios de grau $n$, isto é, monômios que tem comprimento $n$.

Agora, como $A_{n} * A_{m} \subseteq A_{n+m}$, para todos $n, m \geq 1$, temos que $A$ é graduada por grau, ou seja $A$ tem uma estrutura de álgebra graduada (veja Definição 1.2.2).

A álgebra associativa $A=A(X)$ é livre no sentido de que ela satisfaz a propriedade universal definida acima para produto tensorial de espaços vetoriais que é estendida a toda $A$. Isto é, se $B$ é qualquer $K$-álgebra e $\varphi: V \rightarrow B$ é uma aplicação linear, então existe um único homomorfismo de álgebras $\bar{\varphi}: A(X) \rightarrow B$ tal que $\left.\bar{\varphi}\right|_{V}=\varphi$. De fato, vamos supor que $\varphi: V \rightarrow B$ é uma aplicação linear. Então,

$$
\left(x_{1}, \ldots, x_{n}\right) \mapsto \varphi\left(x_{1}\right) \cdots \varphi\left(x_{n}\right)
$$

é uma aplicação multilinear de $V \times \cdots \times V$ em $B$. Ela induz a uma única aplicação linear $\bar{\varphi}: A_{n} \rightarrow B$ que aplica $x_{1} \otimes \cdots \otimes x_{k}$ em $\varphi\left(x_{1}\right) \cdots \varphi\left(x_{k}\right)$. Segue da definição do produto $*$ em $A$ que $\bar{\varphi}$ é um homomorfismo de álgebras.

A partir de agora, denotaremos o produto $x_{1} \otimes \cdots \otimes x_{n}$ por $x_{1} \cdots x_{m}$ e $(u) *(v)$ de $u, v \in A(X)$ simplesmente por $(u)(v)$.

Exemplo 1.2.3 Suponhamos que $X=\{x, y\}$, então

$$
A(X)=\langle 1, \underbrace{x, y}_{\text {grau } 1}, \underbrace{x^{2}, x y, y x, y^{2}}_{\text {grau } 2}, \ldots, \underbrace{x^{n}, x^{n-1} y, \ldots, y^{n}}_{\text {grau } n}, \ldots\rangle_{K} .
$$

Exemplo 1.2.4 Suponhamos que $X=\left\{x_{1}, x_{2}, \ldots, x_{k}\right\}$, então $A=\bigoplus_{n=0}^{\infty} A_{n}, A_{n}$ é gerado como $K$-espaço pelos monômios $x_{i_{1}} \cdots x_{i_{n}}$ de grau $n$, além disso $\operatorname{dim} A_{n}=k^{n}$. 
Os $A_{n}$ 's são chamados de componentes homogêneas de $A$. Esta decomposição pode ser ainda mais refinada como a seguir.

Para cada $n \geq 1$, consideramos a $k$-upla $\alpha=\left(\alpha_{1}, \alpha_{2}, \ldots, \alpha_{k}, 0,0, \ldots, 0\right)$ e denotamos $|\alpha|=\alpha_{1}+\alpha_{2}+\cdots+\alpha_{k}$. Então $A_{n}$ pode ser decomposta na soma direta

$$
A_{n}=\bigoplus_{|\alpha|=n} A_{\alpha}
$$

Os elementos de $A_{\alpha}$ são monômios $x_{i_{1}} x_{i_{2}} \cdots x_{i_{k}}$ tais que $x_{i_{1}}$ aparece $\alpha_{1}$-vezes, $x_{i_{2}}$ aparece $\alpha_{2}$-vezes, $\ldots, x_{i_{k}}$ aparece $\alpha_{k}$-vezes. A $k$-upla $\alpha=\left(\alpha_{1}, \alpha_{2}, \ldots, \alpha_{k}, \ldots\right)$ é chamada multigrau e se $x_{i_{1}} x_{i_{2}} \cdots x_{i_{k}} \in A_{\alpha}$, dizemos que ele é de multigrau $\alpha$. Em particular, se no monômio $x_{i_{1}} x_{i_{2}} \cdots x_{i_{k}}$, cada variável aparece uma única vez, dizemos que este monômio é multilinear de multigrau $\alpha=(1,1, \ldots)$. Assim, $A_{\alpha}$ é o subespaço de $A$ gerado por todos os monômios de multigrau $\alpha=\left(\alpha_{1}, \alpha_{2}, \ldots, \alpha_{k}\right)$. Os $A_{\alpha}$ 's são chamados de componentes multihomogêneas de $A$.

Temos também que $A(X)$ é decomposta na soma

$$
A(X)=\bigoplus_{\alpha} A_{\alpha}, \quad A_{\alpha}=\left\langle x_{i_{1}}, \ldots, x_{i_{m}}\right\rangle_{K} \quad \alpha=\left(\alpha_{1}, \ldots, \alpha_{m}, 0,0, \ldots, 0\right)-\text { multigrau } .
$$

Por exemplo, se $\alpha=(2,3,1)$ e $X=\{x, y, z\}$, então $A_{\alpha}=\left\langle x^{2} y^{3} z, x z x y^{3}, \ldots\right\rangle_{K}$

Claro que se $\alpha=\left(\alpha_{1}, \ldots, \alpha_{m}, 0,0, \ldots, 0\right)$ e $\beta=\left(\beta_{1}, \ldots, \beta_{n}, 0,0, \ldots, 0\right)$ são multigraus, então

$$
A_{\left(\alpha_{1}, \ldots, \alpha_{m}, 0,0, \ldots, 0\right)} A_{\left(\beta_{1}, \ldots, \beta_{n}, 0,0, \ldots, 0\right)} \subseteq A_{\left(\alpha_{1}+\beta_{1}, \ldots, \alpha_{k}+\beta_{k}, 0,0, \ldots, 0\right)},
$$

onde $k=\max \{n, m\}$ e neste caso dizemos que $A=A(X)$ é uma álgebra multigraduada.

\section{Álgebras absolutamente livres}

Seja $\mathcal{V}$ a classe de álgebras lineares, isto é, se $B \in \mathcal{V}$, então existe apenas o produto linear $*: B \times B \rightarrow B$ definido em $B$.

Seja $X=\left\{x_{i} \mid i \in I\right\}$, conjunto não vazio de variáveis. Denotaremos o grupóide livre sobre $X$ por $\Gamma=\Gamma(X)$, que é o conjunto de todos os monômios não associativos nas 
variáveis de $X$ definido por

$$
\Gamma(X)=\left\{x_{i}, x_{i} * x_{j}, x_{i} *\left(x_{j} * x_{k}\right),\left(x_{i} * x_{j}\right) * x_{k}, \ldots\right\}
$$

Temos que

- $x_{i}$ é dito de grau $1 \quad \forall i \in I$;

- $x_{i} * x_{j}$ é dito de grau $2 \forall i, j \in I$;

- $x_{i} *\left(x_{j} * x_{k}\right),\left(x_{i} * x_{j}\right) * x_{k}$ são ditos de grau $3 \forall i, j, k \in I$

Dessa maneira, $\Gamma_{n}$ denotará o conjunto de todos os produtos de $n$ variáveis de $X$, ou seja $\Gamma_{n}=\left\{u\left(x_{i_{1}}, \ldots, x_{i_{n}}\right) \mid x_{i_{j}} \in X\right\}$. Além disso, se $f \in \Gamma_{n}$ e $g \in \Gamma_{m}$, então $(f * g) \in \Gamma_{n+m}$.

O grupóide livre $\Gamma=\Gamma(X)$ é graduado, isto é, $\Gamma=\bigcup_{n=0}^{\infty} \Gamma_{n}$.

Se $\alpha=\left(\alpha_{1}, \alpha_{2}, \ldots\right)$ é multigrau e denotando $|\alpha|=\alpha_{1}+\alpha_{2}+\cdots+\alpha_{n}+\cdots$, então $\Gamma$ é multigraduado com $\Gamma=\bigcup_{\alpha} \Gamma_{\alpha}$. Dessa forma, $\Gamma_{n}=\bigcup_{|\alpha|=n} \Gamma_{\alpha}$.

Agora, sejam $K$ um corpo e $X=\left\{x_{i} \mid i \in I\right\}$ um conjunto não vazio de variáveis. Seja $\Gamma=\Gamma(X)$ o grupóide livre sobre $X$. Introduzimos a álgebra absolutamente livre sobre $X$ cuja base é $\Gamma$, e denotamos por $F=F(X)$. Os elementos de $F$ são chamados polinômios. A multiplicação em $F$ é definida da seguinta maneira: se $f, g \in F$, onde $f=\sum_{u \in \Gamma} \lambda_{u} u$, $g=\sum_{v \in \Gamma} \mu_{v} v$ então

$$
f * g=\sum_{u, v \in \Gamma} \lambda_{u} \mu_{v}(u) *(v)
$$

A álgebra $F(X)$ é livre no seguinte sentido: se $B$ é qualquer álgebra linear e $\left\{b_{i} \mid i \in\right.$ $I\} \subset B$, então existe único homomorfismo $\varphi: F(X) \rightarrow B$ definido por $\varphi\left(x_{i}\right)=b_{i}, \forall i \in I$.

É claro que um homomorfismo definido em $F(X)$ respeita os parênteses. Por exemplo,

$$
\varphi(((x * y) * z) * x)=((\varphi(x) * \varphi(y)) * \varphi(z)) * \varphi(x) .
$$


Naturalmente, $F(X)$ é graduada e multigraduada, onde $F_{n}(X)$ é o espaço gerado por $\Gamma_{n}(X)$ e $F_{\alpha}(X)$ é gerado por $\Gamma_{\alpha}, n \in \mathbb{N}$ e $\alpha$ é multigrau. Desse modo, $F(X)$ se decompõe em somas diretas:

$$
F(X)=\bigoplus_{n=0}^{\infty} F_{n}(X) \quad \text { e } \quad F(X)=\bigoplus_{\alpha} F_{\alpha}(X)
$$

Além disso, para quaisquer naturais $n, m$ e quaisquer $\alpha, \beta$ multigraus, temos

$$
F_{n} * F_{m} \subseteq F_{n+m} \quad \text { e } \quad F_{\alpha} * F_{\beta} \subseteq F_{\alpha+\beta}
$$

\section{Álgebras de Lie livres}

Definição 1.2.5 Seja $X=\left\{x_{i} \mid i \in I\right\}$ um conjunto não vazio de variáveis. Dizemos que o $K$-espaço vetorial $L$ gerado por $X$ é uma álgebra de Lie livre sobre o conjunto $X$ se $L$ é uma álgebra de Lie e, para qualquer álgebra de Lie $B$ e qualquer $\left\{b_{i} \mid i \in I\right\} \subset B$, existe um único homomorfismo $\varphi: L \rightarrow B$ tal que $\varphi\left(x_{i}\right)=b_{i}$.

Recorde que, pela Definição 1.1.2 uma álgebra $L$ é chamada álgebra de Lie se, e somente se, satisfaz

1. $x * x \equiv 0$;

2. $J(x, y, z)=x *(y * x)+y *(z * x)+z *(x * y) \equiv 0$, onde $J$ é a identidade de Jacobi.

Seja $F=F(X)$ a álgebra absolutamente livre sobre o conjunto não vazio de variáveis $X=\left\{x_{i} \mid i \in I\right\}$ e seja $I \subseteq F$ o ideal gerado por todos os elementos de $F(X)$ do tipo $f * f$ e $J(f, g, h), f, g, h \in F$.

Como espaço, temos que

$$
I=\left\langle u_{1} * \cdots u_{s} *(f * f) * u_{s+1} * \cdots * u_{t} ; v_{1} * \cdots v_{p} * J(f, g, h) * v_{p+1} * \cdots * v_{q}\right\rangle_{K},
$$

onde $u_{i}, v_{j}, f, g, h \in F$.

Assim, temos o seguinte resultado.

Lema 1.2.6 ([1], pág. 46) A álgebra quociente $L(X)=F(X) / I$ é uma álgebra de Lie livre sobre o conjunto $X$. 
Demonstração. Primeiro, note que $L(X)$ é, de fato uma álgebra de Lie. Como

$$
L(X)=F(X) / I=\{u+I \mid u \in F\},
$$

temos que,

$$
(u+I) *(u+I)=(u * u)+u * I+I * u+I * I \subset I
$$

$\mathrm{e}$

$$
J(u+I, v+I, w+I)=J(u, v, w)+I \subset I .
$$

Agora, seja $H$ uma álgebra de Lie qualquer e seja $\left\{h_{i} \mid i \in I\right\} \subset H$. Como $F(X)$ é livre, existe um único homomorfismo $\varphi: F(X) \rightarrow H$, dado por $\varphi\left(x_{i}\right)=h_{i}$. Claro que os elementos de $F(X)$ que são da forma $f * f$ e $J(f, g, h)$ são levados no zero. Logo $I \subseteq \operatorname{ker} \varphi$ e pelo Teorema 1.1.11, existe um homomorfismo induzido $\widetilde{\varphi}: L(X)=F(X) / I \rightarrow H$ definido por $\widetilde{\varphi}(u+I)=\varphi(a)$, cujo núcleo é $\operatorname{ker} \varphi / I$.

Lema 1.2.7 A álgebra de Lie livre livremente gerada pelo conjunto não vazio $X=$ $\left\{x_{i} \mid i \in I\right\}$ é única, a menos de isomorfismos.

Demonstração. Recorde que pela propriedade universal de $L(X)$, para toda $H$ álgebra de Lie e para todo $\left\{h_{i} \mid i \in I\right\} \subset H$, existe um único homomorfismo $\varphi: L(X) \rightarrow H$ tal que $\varphi\left(x_{i}\right)=h_{i}$. Suponha que exista outra álgebra de Lie livre sobre $X$, isto é, existe $L_{1}(X)$, então ela deve satisfazer a propriedade universal também para qualquer álgebra de Lie $H$, em particular, para $L(X)$. Ou seja, exite um único homomorfismo $\varphi^{\prime}: L_{1}(X) \rightarrow L(X)$ tal que para todo $\left\{x_{i} \mid i \in I\right\} \subset L(X), \varphi^{\prime}\left(y_{i}\right)=x_{i}$. Da mesma forma, existe único $\varphi: L(X) \rightarrow L_{1}(X)$, tal que para todo $\left\{y_{i} \mid i \in I\right\}, \varphi\left(x_{i}\right)=y_{i}$. Denotando $I_{L(X)}$ e $I_{L_{1}(X)}$ as funções identidades de $L(X)$ e $L_{1}(X)$, respectivamente, temos que $I_{L(X)}\left(x_{i}\right)=x_{i}$ e $I_{L_{1}(X)}\left(y_{i}\right)=y_{i}$. Logo,

$$
I_{L(X)}\left(x_{i}\right)=x_{i}=\varphi^{\prime} \circ \varphi\left(x_{i}\right),
$$

assim como

$$
I_{L_{1}(X)}\left(y_{i}\right)=y_{i}=\varphi \circ \varphi^{\prime}\left(y_{i}\right)
$$

Portanto, $\varphi$ é inverso de $\varphi^{\prime}$. Isto é, $\varphi$ e $\varphi^{\prime}$ são isomorfismos.

Lema 1.2.8 ([1], pág. 46) Seja $L(X)=F(X) / I$ a álgebra de Lie livre. Então $L(X)$ é graduada e multigraduada. 
Demonstração. Para provarmos esta afirmação, primeiro precisamos mostrar que $I$ é um ideal homogêneo e multihomogêneo. Isto é, que $I$ possui as seguintes decomposições, com respeito as decomposições de $F(X)$ :

$$
I=\bigoplus_{n=0}^{\infty}\left(I \cap F_{n}\right) \quad \text { e } \quad I=\underset{\alpha}{\oplus}\left(I \cap F_{\alpha}\right)
$$

onde $\alpha=\left(\alpha_{1}, \ldots, \alpha_{n}, \ldots\right)$.

Como os dois casos são semelhantes, provaremos somente a segunda decomposição. Denotaremos por $I_{\alpha}$ a interseção $I \cap F_{\alpha}$, onde $\alpha=\left(\alpha_{1}, \ldots, \alpha_{n}, \ldots\right)$.

Se $u=\sum_{\alpha} \lambda_{\alpha} u_{\alpha} \in I, u_{\alpha} \in F_{\alpha}, \lambda_{\alpha} \in K$, queremos provar que $u_{\alpha} \in I$. Por definição, $I$ é gerado por $u_{1} * \cdots * u_{s} *(f * f) * u_{s+1} * \cdots * u_{t}$ e por $v_{1} * \cdots * v_{p} * J(f, g, h) * v_{p+1} * \cdots * v_{q}$. Assim, seja $f=\sum_{i=1}^{t} \lambda_{\alpha_{i}} f_{\alpha_{i}}$, então

$$
(f * f)=\sum \lambda_{\alpha_{i}}\left(f_{\alpha_{i}} * f_{\alpha_{i}}\right)+\sum \lambda_{\alpha_{i}} \lambda_{\alpha_{j}}\left(f_{\alpha_{i}} * f_{\alpha_{j}}+f_{\alpha_{j}} * f_{\alpha_{i}}\right)
$$

claro que o produto $f_{\alpha_{i}} * f_{\alpha_{i}} \in F_{2 \alpha_{i}}$ está em $I$. A soma $f_{\alpha_{i}} * f_{\alpha_{j}}+f_{\alpha_{j}} * f_{\alpha_{i}} \in F_{\alpha_{i}+\alpha_{j}}$ é igual a $\left(f_{\alpha_{i}}+f_{\alpha_{j}}\right)\left(f_{\alpha_{i}}+f_{\alpha_{j}}\right)-f_{\alpha_{i}} * f_{\alpha_{i}}-f_{\alpha_{j}} * f_{\alpha_{j}}$ o qual está em $I$.

Sejam agora, $f=\sum_{\alpha} \lambda_{\alpha} f_{\alpha}, g=\sum_{\beta} \mu_{\beta} g_{\beta}, h=\sum_{\gamma} \nu_{\gamma} h_{\gamma}$, então

$$
J(f, g, h)=\sum \lambda_{\alpha} \mu_{\beta} \nu_{\gamma} J\left(f_{\alpha}, g_{\beta}, h_{\gamma}\right)
$$

onde $J\left(f_{\alpha}, g_{\beta}, h_{\gamma}\right) \in I \cap F_{\alpha+\beta+\gamma}$ é homogêneo.

Vamos denotar $L_{n}(X)=\left(F_{n}(X)\right) / I_{n}$ e $L_{\alpha}\left(F_{\alpha}\right) / I_{\alpha}$, onde $n \in \mathbb{N}$ e $\alpha=\left(\alpha_{1}, \ldots, \alpha_{n}, \ldots\right)$. Uma vez que $I$ é homogêneo, vamos ter

$$
L(X)=\bigoplus_{n=0}^{\infty} L_{n}(X) \quad \text { e } \quad L(X)=\bigoplus_{\alpha} L_{\alpha}(X)
$$

\section{3 Álgebra envelopante universal}

Seja $L$ uma álgebra de Lie, sobre um corpo $K$, gerada pelo conjunto não vazio $\left\{v_{i} \mid i \in\right.$ $I$ \} . Recorde que cada álgebra de Lie $L$ sobre um corpo $K$ é um espaço vetorial sobre $K$. 
Seja $T=T(L)$ a álgebra tensorial de $L$ sobre $K$, isto é, $T$ é uma álgebra com base no espaço vetorial $L$ que por definição é

$$
T(L)=\langle 1\rangle \oplus L_{1} \oplus L_{2} \oplus \cdots \oplus L_{n} \oplus \cdots
$$

onde $L_{1}=L$ e $L_{n}=L \otimes L \otimes \cdots \otimes L, n$ vezes.

Neste caso, $T$ tem uma estrutura natural de álgebra associativa com unidade, não comutativa e graduada e é uma álgebra associativa livre gerada por $X$. Seja $I$ o ideal bilateral de $T$ que é gerado por todos os elementos da forma

$$
x \otimes y-y \otimes x-[x, y], \quad x, y \in L
$$

e sejam $U=U(L)$, a álgebra quociente $T / I$ e $i: L \rightarrow U$ a composição $i=p \circ j$, onde $j$ é a inclusão canônica de $L$ em $T$ e $p$ é o homomorfismo canônico de $T$ em $T / I$. Note que, como $\operatorname{ker} p=I$ e pela definição de $I$, temos

$$
p([x, y])=p(x \otimes y-y \otimes x) .
$$

Portanto, para qualquer $x, y \in L$,

$$
\begin{aligned}
i([x, y]) & =p([x, y]) \\
& =p(x \otimes y-y \otimes x) \\
& =p(x) p(y)-p(y) p(x) \\
& =[p(x), p(y)] \\
& =[p \circ j(x), p \circ j(y)] \\
& =[i(x), i(y)] .
\end{aligned}
$$

Logo, $i$ é um homomorfismo de álgebras de Lie da álgebra $L$ em $U(L)^{(-)}$.

O par $(U, i)$ satisfaz a propriedade universal para $L$. De fato, sejam $A$ qualquer álgebra associativa com 1 e $\varphi: L \rightarrow A^{(-)}$um homomorfismo qualquer de álgebras de Lie, então pela propriedade universal da álgebra tensorial $T$, existe uma única extensão de $\varphi$ a um 
homomorfismo de álgebras $\bar{\varphi}: T \rightarrow A$. Agora, se $x, y \in L$, então

$$
\begin{aligned}
\bar{\varphi}(x \otimes y-y \otimes x-[x, y]) & =\varphi(x) \varphi(y)-\varphi(y) \varphi(x)-\varphi([x, y]) \\
& =0 .
\end{aligned}
$$

Portanto, $I \subset \operatorname{ker} \bar{\varphi}$ o que implica que $\bar{\varphi}$ define um homomorfismo de álgebras $\psi$ : $U=T / I \rightarrow A$, dado por $\bar{\varphi}=\psi \circ p$. Agora, para qualquer $x \in L$, temos

$$
\psi \circ i(x)=\psi \circ p \circ j(x)=\bar{\varphi} \circ j(x)=\varphi(x),
$$

o que mostra que o diagrama



é comutativo. O homomorfismo é único porque $i(L)$ gera $U$, uma vez que $L$ gera $T$.

A álgebra quociente $U(L)=T(L) / I$ é chamada de álgebra envelopante universal da álgebra de Lie $L$.

Formalmente, temos a seguinte definição.

Definição 1.3.1 Seja L uma álgebra de Lie sobre um corpo K. Suponha que $i: L \rightarrow$ $U(L)^{(-)}$seja um homomorfismo de álgebras de Lie da álgebra L na álgebra de Lie associada com a K-álgebra associativa $U(L)$. O par $(U(L), i)$ é chamado de álgebra envelopante universal de $L$ se para cada $K$-álgebra associativa $A$ e cada homomorfismo de álgebras de Lie $f: L \rightarrow A^{(-)}$, existe um único homomorfismo $\bar{f}: U(L) \rightarrow$ A tal que o diagrama

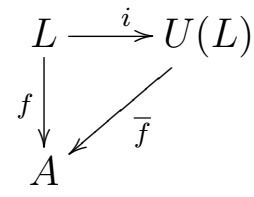

comuta, isto é, $\bar{f} \circ i=f$. 
A aplicacão $i: L \rightarrow U(L)$ é a projeção canônica de $K$-espaços vetoriais definida pela projeção $L \subset T(L) \rightarrow U(L)$ a qual é um homomorfismo de álgebras de Lie dada por

$$
\begin{aligned}
i: L & \rightarrow U(L)^{(-)} \\
l & \mapsto l+I
\end{aligned}
$$

Lembrando que $I$ é o ideal de $T(L)$ gerado pelos elementos da forma $x \otimes y-y \otimes x-[x, y]$, para todos $x, y \in L$, a aplicação $i: L \rightarrow U(L)$ é injetiva e $\langle 1\rangle \cap i(L)=\emptyset$. Assim, temos a seguinte definição.

Definição 1.3.2 Seja L uma álgebra de Lie sobre um corpo $K$ e seja $(U(L), i)$ a sua álgebra envelopante universal. O ideal de $U(L)$ gerado por $i(L)$, a imagem de $L$ por $i$, é chamado o ideal de aumento de $U(L)$ e é denotado por $\omega(L)$.

Equivalentemente a esta definição, temos que $\omega(L)$ é o núcleo do homomorfismo $\epsilon$ : $U(L) \rightarrow K$, chamado de homomorfismo de aumento de $U(L)$.

O próximo resultado é o Teorema de Poincaré-Birkhoff-Witt, o qual não daremos a prova dele aqui porque existem vários livros que dão uma prova para ele, veja por exemplo [10], página 67 à página 69, [11], página 11 e [20], página 159.

Teorema 1.3.3 (Poincaré-Birkhoff-Witt) Seja L uma álgebra de Lie com base dada por $\left\{x_{i} \mid i \in I\right\}$, onde I é um conjunto de indices ordenado. Então $U(L)$, a álgebra envelopante universal de L, tem uma base formada pelos monômios

$$
x_{i_{1}} x_{i_{2}} \cdots x_{i_{k}}, i_{1} \leq i_{2} \leq \ldots \leq i_{1}, i_{j} \in I, k=0,1,2, \ldots
$$

Nos referimos a estes monômios da base de $U(L)$ obtidos a partir do Teorems 1.3.3 como PBW-monômios.

Observe que se $L=\left\langle x_{1}, \ldots, x_{n}\right\rangle_{K}$ é uma álgebra de Lie de dimensão finita, então $U(L)=\left\langle x_{1}^{\alpha_{1}} \cdots x_{n}^{\alpha_{n}} \mid \alpha_{i} \geq 0\right\rangle_{K}$. E se $L=\left\langle x_{1}, \ldots, x_{n}\right\rangle_{K}$ é abeliana, isto é, $[x, y]=0$ para todos $x, y \in L$, então $U(L)$ é isomorfa ao anel de polinômios $K\left[x_{1}, \ldots, x_{n}\right]$.

Lema 1.3.4 A menos de isomorfismos, a álgebra envelopante universal $U(L)$ de uma álgebra de Lie L é única. 
Demonstração. Sejam $(U(L), i)$ e $(V(L), j)$ álgebras envelopantes universais de $L$, então existe um único isomorfismo $h: U(L) \rightarrow V(L)$ tal que $h \circ i=j$. De fato, da propriedade universal de $U(L)$ e $V(L)$ temos a existência de aplicações $f$ e $g$ tais que os diagramas
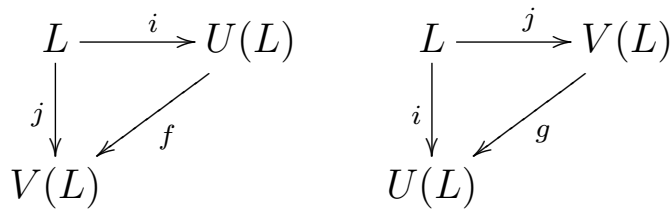

comutam. Consequentemente, temos

$$
(g \circ f) \circ i=g \circ j=i, \quad(f \circ g) \circ j=f \circ i=j .
$$

Portanto, os seguintes diagramas são comutativos
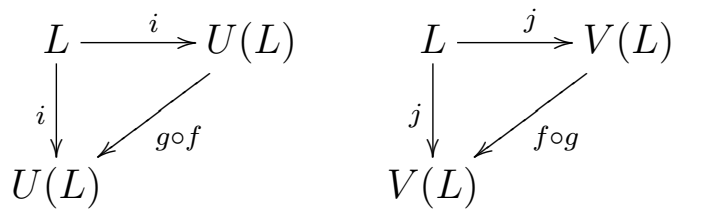

Por outro lado, obtemos diagramas comutativos se considerarmos $i d_{U(L)}$ e $i d_{V(L)}$ ao invés de $g \circ f, f \circ g$, respectivamente. A unicidade da definição implica que $g \circ f=i d_{U(L)}$ e $f \circ g=i d_{V(L)}$. Portanto, $f$ e $g$ são isomorfismos.

Sejam $X=\left\{x_{i} \mid i \in I\right\}$ um conjunto não vazio de variáveis, $L(X)$ a álgebra de Lie livre sobre $X$ e considere $U(L(X))$, a álgebra envelopante universal da álgebra $L(X)$. Já sabemos pelo Lema 1.2.7 e pelo Lema 1.3.4 que $L(X)$ e $U(L(X))$ são únicas (a menos de isomorfismos). Então o próximo resultado é válido.

Lema 1.3.5 (Teorema de Witt, [20], pág. 168) Seja $A(X)$, a álgebra associativa livre gerada por $X$ e seja $H$ a álgebra de Lie $\operatorname{Lie}(X)=\left\langle\left[x_{i_{1}}, x_{i_{2}}, \ldots, x_{i_{n}}\right] \mid x_{i} \in X\right\rangle_{k}$ gerada pelos comutadores dos elementos de X. Então

1. $H$ é isomorfa a $L(X)$, álgebra de Lie livre gerada por $X$; 
2. $A(X) \cong U(L(X))$.

Demonstração. Primeiro, vamos provar 1. Seja $B$ uma álgebra de Lie qualquer e seja $U(B)$ sua álgebra envelopante universal. Queremos mostrar que, para qualquer conjunto $\left\{b_{i} \mid i \in I\right\} \subset B$, existe um homomorfismo $\varphi$ de $H$ em $B$ tal que $\varphi\left(x_{i}\right)=b_{i}$ e que ele é único. Note que, $H=\operatorname{Lie}(X)$ é uma subálgebra de Lie gerada por $X$ de $A(X)^{(-)}$, a álgebra de Lie associada a $A(X)$. Assim, existe a inclusão canônica $j: H \hookrightarrow A(X)$. Pela propriedade universal da álgebra associativa livre $A(X)$, existe um único homomorfismo $\psi$ de $A(X)$ na álgebra $U(B)$, definido por $\psi\left(x_{i}\right)=b_{i}$, onde $b_{i} \in B, i \in I$. A restrição de $\psi$ sobre $H$ é um homomorfismo de $H$ em $U(B)^{(-)}$. Como, $\psi\left(\left[x_{i}, x_{j}\right]\right)=\left[\psi\left(x_{i}\right), \psi\left(x_{j}\right)\right]=$ $\left[b_{i}, b_{j}\right]$ e como $B$ gera $U(B)$, segue que a imagem de $H$ está em $B$ e isto nos dá que $H=\operatorname{Lie}(X)$ é livre na classe das álgebras de Lie.

Agora, considere a afirmação 2. Seja $B$ uma álgebra associativa, e seja $B^{(-)}$a álgebra de Lie associada a $B$, considere também $\varphi: \operatorname{Lie}(X) \rightarrow B^{(-)}$um homomorfismo. A aplicação $\phi: X \rightarrow B$ definida por $\phi(x)=\varphi(x), x \in X$, induz um único homomorfismo $\psi: A(X) \rightarrow B$. Como $\varphi(x)=\psi(x)$, temos que a restrição de $\psi$ sobre $H$ é igual a $\varphi$. Mas esta é a definição de álgebra envelopante universal, o que pelo Lema 1.3.4 nos dá $U(H) \cong A(X)$.

\section{Base de Hall}

A definição que apresentamos a seguir pode ser encontrada em maiores detalhes em [1], na página 48. Seja $X=\left\{x_{i} \mid i \in I\right\}$ um conjunto não vazio de variáveis totalmente ordenado e seja $\Gamma=\Gamma(X)$ o grupóide absolutamente livre sobre $X$ com o produto * Considere o conjunto $R=R(X) \subset \Gamma(X)$.

Definição 1.3.6 Dizemos que $R=R(X)$ é uma família base com a boa ordenação $\geq$, se satisfaz as seguintes condições:

(R1) $X \subset R$;

(R2) $w=u * v \in R$ se, e somente se:

(i) $u, v \in R$;

(ii) $u>v$; 
(iii) se $u=u_{1} * u_{2}$ então $v \geq u_{2}$

(R3) $u * v>v$.

Colocamos $R_{n}=R \cap \Gamma_{n}(X), R_{\alpha}=R \cap \Gamma_{\alpha}$, onde $n \in \mathbb{N}$ e $\alpha=\left(\alpha_{1}, \alpha_{2}, \ldots\right)$ um multigrau.

Podemos construir o conjunto $R(X)$ como um conjunto bem ordenado. Se colocarmos $R_{1}=X$ e inserirmos neste conjunto qualquer boa ordenação, então os conjuntos $R_{n}$, $n=2,3, \ldots$ podem ser bem definidos de maneira que $R_{n+1}$ contenha $R_{n}$ e todos os monômios de grau $n+1$ de $R_{n} * R_{n}$ os quais satisfazem (ii) e (iii). Estendemos a ordenação de $R_{n}$ a $R_{n+1}$ arbitrariamente, mas para ter certeza que qualquer $u \in R_{n+1} \backslash R_{n}$ e $v \in R_{n}$ temos $u>v$. Então, podemos colocar $R=\bigcup_{n=1}^{\infty} R_{n}$. Qualquer família base deste tipo é chamada base se Hall.

Em particular, denotando por $d$ o grau de um monômio $u \in \Gamma(X)$, podemos trocar a condição 3 por:

(R3') se $d(u)>d(v)$ então $u>v$.

Por exemplo, considere o conjunto $X=\{x, y\}$ com $y>x$ e o produto sendo os comutadores de Lie [, ]. Então a base de Hall para este conjunto será

$$
R=R_{1} \cup R_{2} \cup \cdots \cup R_{n} \cup \cdots
$$

onde $R_{n}$ contém monômios de grau $n$, isto é

. $R_{1}: y>x$

- $R_{2}:[y, x]$

- $R_{3}: \underbrace{[[y, x], x]}_{x \geq x}, \underbrace{[[y, x], y]}_{x \leq y} ;$

- $R_{4}:[[[y, x], x], x],[[[y, x], x], y],[[[y, x], y], y]$

- $R_{5}:[[[[y, x], x], x], x],[[[[y, x], x], x], y],[[[[y, x], x], y], y],[[[[y, x], y], y], y]$, $[[[y, x], x],[y, x]],[[[y, x], y],[y, x]]$

. 
Note, por exemplo, que $[[[[y, x], x], x], x],[[[[y, x], x], x], y],[[[[y, x], x], y], y]$, $[[[[y, x], y], y], y]$ são de multigrau $\alpha=(4,1)$, enquanto que os elementos de multigrau $\beta=(3,2)$ são $[[[y, x], x],[y, x]]$ e $[[[y, x], y],[y, x]]$.

Seja $X=\left\{x_{i} \mid i \in I\right\}$ um conjunto não vazio de variáveis totalmente ordenado e seja $L=L(X)$ a álgebra de Lie livre sobre $X$. Considere a multigraduação de $L$, isto é, a decomposição na soma direta $L=\bigoplus_{\alpha} L_{\alpha}$. Então, com respeito a base de Hall, como foi construída acima, vale o seguinte resultado.

Lema 1.3.7 ([1], pág. 48) Para todo $\alpha$-multigrau fixo, $L_{\alpha}=\left\langle R_{\alpha}\right\rangle_{K}$.

Demonstração. A prova é feita por indução sobre $n=|\alpha|$. Considere a base de indução $|\alpha|=1$. Então, neste caso, $R_{\alpha}=\Gamma_{\alpha}=\left\{x_{\alpha}\right\}$-letra e não há nada a fazer. Agora, vamos supor $|\alpha|>1$. Qualquer elemento em $L_{\alpha}$ é combinação linear de elementos da forma $w=[u, v]$, onde $u \in L_{\beta}$ e $v \in L_{\gamma} \operatorname{com} \beta+\gamma=\alpha$. Por indução, $u \in\left\langle R_{\beta}\right\rangle_{K}$ e $v \in\left\langle R_{\gamma}\right\rangle_{K}$. Por distributividade, podemos nos restringir ao caso quando $w=[u, v]$ e $u \in R_{\beta}, v \in R_{\gamma}$ com $\beta+\gamma=\alpha$ e $u>v$ (usando a anticomutatividade). Continuaremos a prova, usando indução sobre $|\beta|$, para provar que $w$ como acima é uma combinação linear de monômios da base $w_{1}=\left[u_{1}, v_{1}\right], \ldots, w_{s}=\left[u_{s}, v_{s}\right]$, onde $w_{i}>v, i=1,2, \ldots, s$.

Se $|\beta|=1$, então $u \in R_{\beta}=x_{\beta}$-letra e $u>v$, onde $v$ é a palavra vazio $(|\gamma|=0)$, por (iii) se $u=u_{1} * u_{2}$ então $w \in R_{\alpha}$. Não temos que verificar nada. Suponhamos que $|\beta| \geq 2$. Então $w=\left[\left[u_{1}, u_{2}\right], v\right]$. Temos duas situações a considerar: $u_{2} \leq v$ e $u_{2}>v$.

- se $u_{2} \leq v$, então (iii) é válido, o que implica que $w \in R_{\alpha}$ (por definição);

- se $u_{2}>v$, então pela identidade de Jacobi

$$
\begin{aligned}
w & =\left[\left[u_{1}, u_{2}\right], v\right] \\
& =-\left[\left[v, u_{1}\right], u_{2}\right]-\left[\left[u_{2}, v\right], u_{1}\right] \\
& =\left[\left[u_{1}, v\right], u_{2}\right]-\left[\left[u_{2}, v\right], u_{1}\right]
\end{aligned}
$$

Por indução, os monômios $\left[u_{1}, v\right]$ e $\left[u_{2}, v\right]\left(v<u_{2}<u_{1}\right)$ podem ser escritos como combinação linear de monômios da base $u^{\prime}$ tais que $u^{\prime}>v$.

Assim, por indução, verificamos que se $w=[u, v] u, v \in R, u>v$ isto implica que $w$ é combinação linear de monômios $w^{\prime} \in R$ tais que $w^{\prime}=\left[u^{\prime}, u_{2}\right] \in R \operatorname{com} w^{\prime}>u_{2}>v$. 
Recorde que, pelo Lema 1.3.5, a álgebra envelopante universal $U(L(X))$ da álgebra de Lie livre $L(X)$, é isomorfa a $A(X)$. Pelo lema anterior, obtemos $L(X)=\langle R(X)\rangle_{K}$. Agora, pelo Teorema 1.3.3 (Teorema de Poincaré-Birkhoff-Witt), segue o próximo lema.

Lema 1.3 .8 ([1], pág. 49) A álgebra associativa livre $A(X)$ sobre o conjunto $X$ totalmente ordenado, é gerada como espaço pelos monômios da forma

$$
w_{1} w_{2} \cdots w_{s}, \quad w_{1} \leq w_{2} \leq \cdots \leq w_{s}, \quad s \geq 0, w_{i} \in R(X)
$$

Assim, podemos destacar o seguinte resultado.

Lema 1.3.9 ([1], pág. 50) Todo monômio $a=x_{i_{1}} \cdots x_{i_{n}} \in A(X)$, admite único reordenamento em colchetes como em (1.3).

Demonstração. Primeiro, vamos provar a existência. Suponha que na palavra $a=x_{i_{1}} \cdots x_{i_{n}}$ tenhamos que $x_{i_{1}} \cdots x_{i_{n}}=x_{i_{1}} w_{2} \cdots w_{s}$, com $w_{2} \leq w_{3} \leq \cdots \leq w_{s}$ e $w_{i} \in R$. Então temos dois casos a considerar: $x_{i_{1}} \leq w_{2}$ e $x_{i_{1}} \geq w_{2}$. Se $x_{i_{j}} \leq w_{2}$, então já está feito. Suponha que $x_{i_{1}}>w_{2}$. Considere $p$ o maior número inteiro positivo tal que $w^{\prime}=\left[\left[x_{i_{1}}, w_{2}\right], \ldots, w_{p}\right] \in R$, $\left(\left[x_{i_{1}}, w_{2}\right] \in R\right)$, então $a=w^{\prime} w_{p+1} \cdots w_{s}$ e $w_{p} \leq w_{p+1}$ o que implica que $w^{\prime} \leq w_{p+1}$ (caso contrário, se $w^{\prime}>w_{p+1}$ então $\left[x_{i_{1}}, \ldots, w_{p+1}\right] \in R$ e $p$ não seria o maior). Assim, a existência está provada.

Agora, vamos supor que $a=x_{i_{1}} \cdots x_{i_{n}}$ admite duas reordenações, isto é, $w_{1} \cdots w_{s}$ e $w_{1}^{\prime} \cdots w_{r}^{\prime}$, onde $w_{1}=\left[\left[x_{i_{1}}, a_{1}\right], a_{2}, \ldots, a_{l}\right]$ com $a_{1} \leq \ldots \leq a_{l}$ e $w_{1}^{\prime}=\left[\left[x_{i_{1}}, b_{1}\right], b_{2}, \ldots, b_{m}\right]$ com $b_{1} \leq \ldots \leq b_{m}$. Denotando $a^{\prime}=x_{i_{2}} \cdots x_{i_{n}}$, então $a^{\prime}=a_{1} a_{2} \cdots a_{l} w_{2} \cdots w_{s}$ e $a^{\prime}=$ $b_{1} b_{2} \cdots b_{m} w_{2}^{\prime} \cdots w_{r}^{\prime}$. Pela propriedade da base, temos $a_{l}<w_{1} \leq w_{2} \leq \ldots \leq w_{s}$ e $b_{m}<$ $w_{1}^{\prime} \leq w_{2}^{\prime} \leq \ldots \leq w_{r}^{\prime}$. Então $a^{\prime}$ é únicamente reordenado como queremos. Para provar isso, é suficiente ver que $a_{l} \leq w_{2}$ e $b_{m} \leq w_{2}^{\prime}$. Mas este é o caso, pois aplicando a condição (R3), temos $a_{l} \leq w_{1} \leq w_{2}$, assim como também $b_{m} \leq w_{1}^{\prime} \leq w_{2}^{\prime}$. Pela hipótese de indução aplicado a $a^{\prime}$, teremos $a_{1}=b_{1}, \ldots, a_{l}=b_{m}, w_{2}=w_{2}^{\prime}, w_{s}=w_{r}^{\prime}$ e devemos ter $l=m$ pois caso contrário, teríamos $\left[\left[x_{i_{1}}, a_{1}\right], a_{2}, \ldots, a_{l}\right] \in R$ com $l$ maior com essa propriedade, mas também teríamos $\left[\left[x_{i_{1}}, a_{1}\right], a_{2}, \ldots, a_{m}\right] \in R$ com $m$ também maior com essa propriedade, o que é uma contradição. Portanto, $l=m$ e $s=r$. Agora, como $a_{l}$ é o último tal que $\left[\left[x_{i_{1}}, a_{1}\right], a_{2}, \ldots, a_{l}\right] \in R$, temos provado a unicidade. 
Teorema 1.3.10 ([1], pág. 51) Seja $X$ um conjunto não vazio e $L(X)$ a álgebra de Lie livre sobre $X$. Os monômios da base $R(X)$ formam uma base de $L(X)$ como espaço vetorial.

Demonstração. Considere $\alpha$-multigrau qualquer fixo. Temos que, se $A=(X)$ é a álgebra associativa livre, então $A_{\alpha}$ é gerado como $K$-espaço pelos monômios $a=x_{i_{1}} \cdots x_{i_{n}}$ de multigrau $\alpha, \operatorname{dim} A_{\alpha}<\infty$. Mas pelo Lema 1.3.8, $A_{\alpha}$ é gerado pelos monômios do tipo $w_{1} \cdots w_{s}, w_{i} \in R$, com $w_{1} \leq \cdots \leq w_{s}$ e multigrau $\alpha$. O Lema 1.3.9 diz que o número destes elementos é o mesmo, o que significa que $\left\{w_{1} \cdots w_{s} \mid w_{i} \in R, w_{1} \leq \ldots w_{s}\right\}$ são linearmente independentes. Isto implica que $\left\{w_{i} \mid w_{i} \in R\right\}$ são linearmente independentes.

\section{Identidades}

Todas essas construções nos possibilitam definir o conceito de identidade de álgebras associativas e de Lie sobre um corpo $K$. Isto é, qualquer equação da forma $f \equiv 0$, sendo $f \in A(X)(f \in L(X))$ um polinômio não trivial, onde $A(X)$ é uma álgebra associativa livre $(L(X)$ é uma álgebra de Lie livre) sobre $K$. Esta identidade é satisfeita por uma álgebra $B$ associativa (de Lie) se, e somente se, para qualquer homomorfismo $\varphi: A(X) \rightarrow B$ $(\varphi: L(X) \rightarrow B)$ temos $\varphi(f)=0$. A parte da não trivialidade vem do fato de $f$ ser não nulo.

Equivalentemente, temos a seguinte definição.

Definição 1.3.11 Seja A uma K-álgebra e $f=f\left(x_{1}, x_{2}, \ldots, x_{n}\right) \in A(X)(f \in L(X))$. Dizemos que $f \equiv 0$ é uma identidade polinomial de $A$ se $f\left(a_{1}, a_{2}, \ldots, a_{n}\right)=0$, para quaisquer $a_{1}, a_{2}, \ldots, a_{n} \in A$.

Diremos usualmente que $f \equiv 0$ é uma identidade para $A$ ou que $A$ satisfaz $f \equiv 0$. Como o polinômio identicamente nulo $f=0$ é uma identidade para qualquer álgebra $A$, fazemos a definição a seguir.

Definição 1.3.12 Se A satisfaz uma identidade polinomial não trivial $f \equiv 0$, então dizemos que A é uma PI-álgebra.

Daremos alguns exemplos de PI-álgebras. 
Exemplo 1.3.13 Se A é uma álgebra comutativa, então A é uma PI-álgebra que satisfaz a identidade $[x, y] \equiv 0$.

Exemplo 1.3.14 Qualquer álgebra nilpotente é uma PI-álgebra. De fato se, $A^{n+1}=0$, para algum $n>1$, então $x_{1} \cdots x_{n+1} \equiv 0$ é uma identidade polinomial para $A$.

Exemplo 1.3.15 Seja A uma álgebra nil de expoente limitado. Isto significa que existe um inteiro $n \geq 1$ tal que $a^{n}=0$, para todo $a \in A$. Então claramente $x^{n} \equiv 0$ é uma identidade polinomial de A.

Exemplo 1.3.16 Seja A uma álgebra associativa de dimensão finita e seja $\operatorname{dim} A=n<$ $\infty$. Então A satisfaz a identidade standard de grau $n$

$$
\operatorname{St}_{n}\left(x_{1}, \ldots, x_{n}\right)=\sum_{\sigma \in S_{n}}(\operatorname{sign} \sigma) x_{\sigma(1)} \cdots x_{\sigma(n)} \equiv 0
$$

onde $S_{n}$ é o grupo simétrico de grau $n$.

Temos ainda o Teorema de Amitsur-Levitzki.

Exemplo 1.3.17 (Teorema de Amitsur-Levitzki, [11], pág. 79) Seja $M_{k}(K)$ a álgebra das matrizes $k \times k$ com entrada em $K$. Então $M_{k}(K)$ satisfaz a identidade standard de grau $2 k$

$$
\mathrm{St}_{2 k}\left(x_{1}, \ldots, x_{2 k}\right) \equiv 0
$$

Podemos definir álgebras de Lie nilpotentes e solúveis em termos de identidades de $L(X)$.

Definição 1.3.18 Seja L uma álgebra de Lie sobre um corpo K. Se L satisfaz a identidade

$$
\left[x_{1}, x_{2}, \ldots, x_{s+1}\right]=\left[\ldots\left[\left[x_{1}, x_{2}\right], x_{3}\right], \ldots, x_{s+1}\right] \equiv 0
$$

e L não satisfaz relação de tamanho menor, dizemos que L é nilpotente de classe s. 
Agora, definindo por indução os monômios a seguir:

$$
\begin{gathered}
\delta_{1}\left(x_{1}, x_{2}\right)=\left[x_{1}, x_{2}\right] \\
\delta_{2}\left(x_{1}, x_{2}, x_{3}, x_{4}\right)=\left[\delta_{1}\left(x_{1}, x_{2}\right), \delta_{1}\left(x_{3}, x_{4}\right)\right]=\left[\left[x_{1}, x_{2}\right],\left[x_{3}, x_{4}\right]\right] \\
\vdots \\
\delta_{n}\left(x_{1}, \ldots, x_{2^{n}}\right)=\left[\delta_{n-1}\left(x_{1}, \ldots, x_{2^{n-1}}\right), \delta_{n-1}\left(x_{2^{n-1}+1}, \ldots, x_{2^{n}}\right)\right]
\end{gathered}
$$

temos a seguinte definição.

Definição 1.3.19 Seja L uma álgebra de Lie sobre um corpo K. Se L satisfaz a identidade

$$
\delta_{c}\left(x_{1}, \ldots, x_{2^{c}}\right) \equiv 0
$$

e não satisfaz outra relação para $k<c$, então dizemos que $L$ é solúvel de classe $c$.

\section{A Lie nilpotência (forte) e a Lie solubilidade (forte) em álgebras associativas}

Seja $A$ uma álgebra associativa arbitrária e considere o produto comutador de Lie em $A$ como $[x, y]=x y-y x, x, y \in A$, recorde que este produto satisfaz os axiomas da Definição 1.1.2. Para cada inteiro positivo $m$, denote por $\gamma_{m}(A)$ o $m$-ésimo termo da série central inferior de $A$, i.e., $\gamma_{1}(A)=A$ e $\gamma_{n+1}(A)=\left[\gamma_{n}(A), A\right], n \geq 1$. Isto nos dá uma sequência decrescente de ideais

$$
A=\gamma_{1}(A) \supseteq \gamma_{2}(A)=[A, A] \supseteq \gamma_{3}(A) \supseteq \cdots
$$

A álgebra $A$ é dita Lie nilpotente se, para algum inteiro positivo $n$, temos $\gamma_{n}(A)=0$. Dizemos que $A$ é Lie nilpotente de classe $s$ se $\gamma_{s+1}(A)=0$ mas $\gamma_{s}(A) \neq 0$.

Definimos também as chamadas potências de Lie superiores como a seguir. A cada passo nós pegamos o ideal gerado pelos comutadores, isto é, colocamos $A^{(0)}=A$ e $A^{(n)}=$ $\left[A^{(n-1)}, A\right] \cdot A$ para todo $n \geq 1$. Também ganhamos uma sequência decrescente de ideais de $A$. 
Assim, dizemos que uma álgebra associativa $A$ é Lie nilpotente forte se, para algum inteiro positivo $n$, temos $A^{(n)}=0$. Dizemos que $A$ é Lie nilpotente forte de classe $d$ quando $A^{(d)} \equiv 0$ e não satisfaz condição semelhante para $d-1$.

Observe também que

$$
\gamma_{n}(A) \subseteq A^{(n-1)}, n \geq 1
$$

Isto significa que a Lie nilpotência forte de classe $d$ implica a Lie nilpotência de mesma classe $d$. A nilpotência de classe 1 é equivalente a nilpotência forte de classe 1 e é equivalente ao fato de $A$ ser uma álgebra de Lie abeliana.

Agora, para cada inteiro positivo $m$, denotaremos por $\delta_{m}(A)$ o $m$-ésimo termo da série derivada de $A$, i.e., $\delta_{0}(A)=A, \delta_{n+1}(A)=\left[\delta_{n}(A), \delta_{n}(A)\right], n \geq 0$.

A álgebra $A$ é dita Lie solúvel se, para algum inteiro positivo $n$, temos $\delta_{n}(A)=0$. Dizemos que $A$ é Lie solúvel de classe $s$ se, e somente se, $\delta_{s}(A)=0$, mas $\delta_{s-1}(A) \neq 0$. Uma outra condição equivalente é que existe a cadeia dos ideais de Lie

$$
A=A_{0} \supset A_{1} \supset \cdots \supset A_{s}=0
$$

tais que os fatores $A_{i} / A_{i+1}, i=0, \ldots, s-1$, são abelianos e $s$ é minimal.

Definimos também as chamadas séries derivadas superiores como a seguir. Colocamos $\widetilde{\delta}_{0}(A)=A, \widetilde{\delta}_{n+1}(A)=\left[\widetilde{\delta}_{n}(A), \widetilde{\delta}_{n}(A)\right] \cdot A, n \geq 0$.

Assim, dizemos que $A$ é Lie solúvel forte se, para algum inteiro positivo $n$, temos $\widetilde{\delta}_{n}(A)=0$. Dizemos que $A$ é Lie solúvel forte de classe $s$ se, e somente se, $\widetilde{\delta}_{s}(A)=0$, mas $\widetilde{\delta}_{s-1}(A) \neq 0$. Uma outra condição equivalente é que existe a cadeia dos ideais

$$
A=A_{0} \supset A_{1} \supset \cdots \supset A_{s}=0
$$

tais que os fatores $A_{i} / A_{i+1}, i=0, \ldots, s-1$, são abelianos e $s$ é minimal.

Observe também que

$$
\delta_{s}(A) \subseteq \widetilde{\delta}_{s}(A), \quad s \geq 0
$$

Em particular, a solubilidade forte de classe $d$ implica a solubilidade de classe $d$. A solubilidade de classe 1 é equivalente a solubilidade forte de classe 1 e é equivalente ao fato de $A$ ser uma álgebra de Lie abeliana. 


\section{Filtração crescente e álgebra graduada associada}

Definição 1.3.20 Uma álgebra $U$ é dita filtrada se, para cada inteiro não negativo $i$, está definido um subespaço $U_{i}$ tal que

1. $U_{n} \subset U_{m}$ se $n \leq m$;

2. $U_{n} \cdot U_{m} \subseteq U_{n+m}, \forall n, m \geq 0$;

3. $\bigcup_{n \geq 0} U_{n}=U$;

Uma importante noção associada com a álgebra filtrada $U$ é a álgebra graduada associada $\bar{U}=\operatorname{gr}(U)$. Obtemos esta álgebra formando o espaço vetorial

$$
\operatorname{gr}(U)=\bar{U}=\bigoplus_{i=0}^{\infty} \bar{U}_{i}, \quad \text { onde } \bar{U}_{i}=U_{i} / U_{i-1}, \quad \text { e } U_{-1}=0 .
$$

A multiplicação em $\bar{U}$ é definida, componente a componente, por

$$
\left(v_{i}+U_{i-1}\right)\left(v_{j}+U_{j-1}\right)=v_{i} v_{j}+U_{i+j-1}
$$

onde $v_{i} \in U_{i}$ e $v_{j} \in U_{j}$. Além disso, se $v_{i} \equiv u_{i} \bmod U_{i-1}$ e $v_{j} \equiv u_{j} \bmod U_{j-1}$ então $v_{i} v_{j} \equiv u_{i} u_{j} \bmod U_{i+j-1}$.

Agora, seja $L$ uma álgebra de Lie e seja $U=U(L)$, sua envelopante universal. Definimos a seguinte cadeia crescente de subespaços de $U$ por

$$
U_{0}=\langle 1\rangle, \quad U_{i}=\langle 1\rangle+L+L^{2}+\cdots+L^{i}, \quad i \geq 1
$$

onde $L^{i}$ é o subespaço de $U(L)$ gerado por todos os produtos de $i$ elementos de $L$. A sequência de subespaços $\left\{U_{n} \mid n \geq 0\right\}$, definida acima, é uma filtração para $U(L)$. De fato,

$$
\begin{aligned}
U_{n} \cdot U_{m} & =\left(\langle 1\rangle+L+\cdots+L^{n}\right) \cdot\left(\langle 1\rangle+L+\cdots+L^{m}\right) \\
& \subset\langle 1\rangle+\cdots+L^{n+m}=U_{n+m}, \quad \forall m, n \geq 0 .
\end{aligned}
$$

Além disso, pelo Teorema 1.3.3, os produtos da forma $v_{i_{1}} v_{i_{2}} \cdots v_{i_{n}}$ geram $U_{n}$, para cada $n \geq 0$. Dessa maneira, temos $U(L)=\bigcup_{n \geq 0} U_{n}$. 
Definição 1.3.21 A filtração $\left\{U_{n} \mid n \geq 0\right\}$ é chamada de filtração crescente de $U(L)$.

Seja $\bar{U}=\operatorname{gr} U(L)$ a álgebra graduada associada. Pelo Teorema 1.3.3, $L$ gera $U$, então o quociente $\bar{L}=U_{1} / U_{0}=(\langle 1\rangle+L) /\langle 1\rangle$ gera a álgebra $\bar{U}$. Se $\left\{v_{i} \mid i \in I\right\}, I$ ordenado, é uma base para $L$, então as classes laterais $\bar{v}_{i}=v_{i}+\langle 1\rangle$ em $\bar{U}_{1}$ geram $\bar{U}$. Agora, sejam $\bar{v}_{i}$ e $\bar{v}_{j}$ então $\bar{v}_{i} \bar{v}_{j}=v_{i} v_{j}+U_{1}, \bar{v}_{j} \bar{v}_{i}=v_{j} v_{i}+U_{1}$ e como $v_{i} v_{j}-v_{j} v_{i}=\left[v_{i}, v_{l}\right] \in U_{1}$, segue que $\bar{v}_{i} \bar{v}_{j}=\bar{v}_{j} \bar{v}_{i}$.

Assim, os geradores comutam e consequentemente $\bar{U}$ é uma álgebra comutativa. Segue que cada elemento de $\bar{U}$ é uma combinação linear dos elementos $\overline{1}=1, \bar{v}_{i_{1}} \bar{v}_{i_{2}} \cdots \bar{v}_{i_{n}}, i_{1} \leq$ $i_{2} \leq \ldots \leq i_{n}$. Pelo Teorema 1.3.3, os diferentes monômios indicados aqui, formam uma base para $\bar{U}$. Isto significa que os $\bar{v}_{i}$ 's são algebricamente independentes e $\bar{U}=\operatorname{gr}(U(L))$ é a álgebra dos polinômios nesses elementos, isto é

$$
\operatorname{gr}(U(L)) \cong K\left[v_{1}, v_{2}, \ldots\right]
$$

Recordando que qualquer álgebra de Lie $L$ sobre um corpo $K$ é um espaço vetorial, temos a definição a seguir.

Definição 1.3.22 A álgebra simétrica de um espaço vetorial $L$ sobre um corpo $K$ é o quociente da álgebra tensorial $T(L)$ pelo ideal $J$ gerado por todos os elementos do tipo $x \otimes y-y \otimes x$, para todos $x, y \in L$. A álgebra simétrica $T(L) / J$ é denotada por $S(L)$.

Nós identificamos $S(L)$, a álgebra simétrica da álgebra de Lie $L$, com a álgebra graduada $\operatorname{gr}(U(L))$ associada construída a partir da filtração crescente de $U(L)$.

\section{Álgebras de Lie restritas. Superálgebras de Lie}

Agora, iremos apresentar outras duas estruturas da teoria de álgebra de Lie que são as álgebras de Lie restritas e as superálgebras de Lie. Antes, iremos mencionar um resultado muito útil para a construção da envelopante restrita que será apresentada a seguir.

Seja $\left\{v_{i} \mid i \in I\right\}$ uma base ordenada de $L$ e suponha que para cada $v_{i}$, existem um inteiro positivo $k_{i}$, um elemento $z_{i}$ do centro de $U(L)$ e um elemento $u_{i} \in U_{k_{i}-1}$ tais que

$$
v_{i}^{k_{i}}=u_{i}+z_{i}
$$


Então vale o seguinte resultado.

Lema 1.3.23 ([51], pág. 58) Os elementos da forma

$$
v_{i_{1}}^{\beta_{1}} v_{i_{2}}^{\beta_{2}} \cdots v_{i_{r}}^{\beta_{r}} z_{i_{1}}^{\gamma_{1}} z_{i_{2}}^{\gamma_{2}} \cdots z_{i_{r}}^{\gamma_{r}}
$$

onde $i_{1}<\ldots<i_{r}, 0 \leq \beta_{j}<k_{j}, \gamma_{j} \geq 0$ e $r=1,2, \ldots$, formam uma base para $U(L)$.

Definição 1.3.24 Seja L uma álgebra de Lie sobre um corpo $K$ de caracterítica $p>0$. Suponhamos que em L existe uma aplicação

$$
\begin{aligned}
{[p]: L } & \rightarrow L \\
x & \mapsto x^{[p]}
\end{aligned}
$$

chamada p-aplicação, que satisfaz:

1. $(\alpha x)^{[p]}=\alpha^{p} x^{[p]}, x \in L, \alpha \in K$;

2. $\operatorname{ad}\left(x^{[p]}\right)=(\operatorname{ad} x)^{p}, \quad x \in L$;

3. $(x+y)^{[p]}=x^{[p]}+y^{[p]}+\sum_{i=1}^{p-1} s_{i}(x, y), \quad x, y \in L$.

onde $i s_{i}(x, y)$ é o coeficiente de $\lambda^{i-1}$ em $x\left(\operatorname{ad}(\lambda x+y)^{p}\right)$. Então o par $(L,[p])$ é chamado álgebra de Lie restrita, ou p-álgebra de Lie.

A origem da noção de álgebra de Lie restrita, vem da seguinte observação: seja $A$ uma álgebra associativa sobre um corpo de característica $p>0$. Então, a álgebra de Lie $A^{(-)}$associada a álgebra $A$ e a aplicação $x \mapsto x^{p}, x \in A$ (a potência ordinária dentro de $A)$ satisfazem os axiomas acima.

As noções de subálgebras, ideais, morfismos de Lie restrita são padrões e alguns deles serão catalogadas a seguir sem mais comentários.

Definição 1.3.25 Seja $(L,[p])$ uma álgebra de Lie restrita. Dizemos que a subálgebra $H \subset L$ (ideal $I \triangleleft L)$ é uma subálgebra restrita (ideal restrito) de $L$ se, e somente se,

$$
x^{[p]} \in H, \quad \forall x \in H \quad\left(x^{[p]} \in I, \quad \forall x \in I\right) .
$$


Definição 1.3.26 Sejam $\left(L_{1},[p]_{1}\right),\left(L_{2},[p]_{2}\right)$ álgebras de Lie restritas sobre um corpo $K$. Uma aplicação $\varphi: L_{1} \rightarrow L_{2}$ é chamada restrita (ou p-homomorfismo) se $\varphi$ é um homomorfismo de álgebras de Lie e $\varphi\left(x^{[p]_{1}}\right)=(\varphi(x))^{[p]_{2}}$, para todo $x \in L_{1}$.

Proposição 1.3 .27 ([51], pág. 67) Seja $(L,[p])$ uma álgebra de Lie restrita. Suponha que $\varphi: L \rightarrow G$ é um homomorfismo de álgebras de Lie, da álgebra de Lie L na álgebra de Lie $G$, tal que $\operatorname{ker}(\varphi)$ é um ideal restrito. Então existe uma única p-aplicação sobre $\varphi(L)$ tal que $\varphi: L \rightarrow \varphi(L)$ é um p-homomorfismo.

Este resultado é importante, pois se $I$ é um ideal restrito de uma álgebra de Lie restrita $L$, então a definição $(x+I)^{[p]}:=x^{[p]}+I$ faz de $L / I$ uma álgebra de Lie restrita tal que o homomorfismo canônico $\pi: L \rightarrow L / I$ é um $p$-homomorfismo.

Agora, iremos introduzir para álgebras de Lie restritas, o conceito análogo como para álgebras de Lie ordinárias, que é a noção de álgebra envelopante universal com a propriedade de "restricidade".

Definição 1.3.28 Seja $(L,[p])$ uma álgebra de Lie restrita sobre um corpo $K$ de característica positiva p. Um par $(u(L), i)$, consistindo de uma álgebra associativa sobre o corpo $K$ com unidade e um homomorfismo restrito $i: L \rightarrow u(L)^{(-)}$, é chamado de álgebra envelopante universal restrita se, dada qualquer álgebra associativa sobre $K$ com unidade e qualquer homomorfismo $\varphi: L \rightarrow A^{(-)}$, existe um único homomorfismo $\bar{\varphi}: u(L) \rightarrow A$ de álgebras associativas tal que $\varphi=\bar{\varphi} \circ i$.

A existência de $u(L)$ pode ser comprovada da seguinte forma.

Seja $\left\{v_{i} \mid i \in J\right\}$ uma base ordenada da álgebra de Lie restrita $(L,[p])$. Aplicamos o Lema 1.3.23, considerando $k_{i}=p$ e escolhendo os elementos do tipo $z_{i}=v_{i}^{p}-v_{i}^{[p]}$. A derivação interna destes elementos é trivial, isto é, pelo axioma 3 da Definição 1.3.24, temos

$$
\begin{aligned}
\operatorname{ad} z_{i} & =\operatorname{ad} v_{i}^{p}-\operatorname{ad} v_{i}^{[p]} \\
& =\left(\operatorname{ad} v_{i}\right)^{p}-\left(\operatorname{ad} v_{i}\right)^{p}=0
\end{aligned}
$$

Isto implica que $z_{i}$ é um elemento central de $U(L)$, isto é, $z_{1}, \ldots, z_{n} \in Z(U(L))$. Além disso, $v_{i}^{[p]} \in L=U_{1} \subset U_{p-1}$. Seja $I$ o ideal de $U(L)$, gerado por todos os elementos 
$z_{i}=v_{i}^{p}-v_{i}^{[p]}$. Por $z_{i} \in Z(U(L))$, o ideal $I$ é bilateral. Pelo Lema 1.3.23, $I$ é gerado por todos os elementos da forma $v_{j_{1}}^{\beta_{1}} \cdots v_{j_{n}}^{\beta_{n}} z_{j_{1}}^{\gamma_{1}} \cdots z_{j_{n}}^{\gamma_{n}}$, onde $j_{1}<\ldots<j_{n}, 0 \leq \beta_{k}<p, \gamma_{j_{k}} \geq 0$, $k=1,2, \ldots, n, \quad n \geq 0$.

Então os elementos

$$
i\left(v_{j_{1}}^{\beta_{1}}\right) i\left(v_{j_{2}}^{\beta_{2}}\right) \cdots i\left(v_{j_{n}}^{\beta_{n}}\right), \quad j_{1}<\ldots<j_{n}, 0 \leq \beta_{k}<p, k=1,2, \ldots, n, \quad n \geq 0
$$

formam uma base para $U(L) / I$. Então, definimos $u(L):=U(L) / I$ e $i(x)=x+I$, para cada $x \in L$.

De fato, $(u(L), i)$ definida acima satisfaz a propriedade universal. Primeiro, seja $\sum \alpha_{j} v_{j} \in L$, então a aplicacão $L \rightarrow U(L)$ que pega $x \in L$ e leva em $x^{p}-x^{[p]}$ é tal que

$$
\left(\sum \alpha_{j} v_{j}\right)^{p}-\left(\sum \alpha_{j} v_{j}\right)^{[p]}=\sum \alpha_{j}^{p}\left(v_{j}^{p}-v_{j}^{[p]}\right) \equiv 0(\bmod I) .
$$

Agora, seja $A$ uma álgebra associativa sobre $K$ e seja $\varphi: L \rightarrow A^{(-)}$um homomorfismo tal que $\varphi\left(x^{[p]}\right)=(\varphi(x))^{p}$ para todo $x \in L$. Então, $\varphi$ possui a única extensão $\bar{\varphi}: U(L) \rightarrow A$ tal que

$$
\begin{aligned}
\bar{\varphi}\left(z_{j}\right) & =\bar{\varphi}\left(v_{j}^{p}-v_{j}^{[p]}\right) \\
& =\bar{\varphi}\left(v_{j}^{p}\right)-\bar{\varphi}\left(v_{j}^{[p]}\right) \\
& =\varphi\left(v_{j}^{p}\right)-\varphi\left(v_{j}^{[p]}\right) \\
& =0, \quad \text { para todo } j \in J
\end{aligned}
$$

Dessa maneira, $\bar{\varphi}(I)=0$ e obtemos o homomorfismo induzido $\psi: U(L) / I \rightarrow A$, definido por $\psi(a+I)=\varphi(a)$. Como $i(L)$ gera $U(L), \psi$ é unicamente determinado por $\varphi=\psi \circ i$.

Definição 1.3.29 Uma superálgebra de Lie é um espaço vetorial $\mathbb{Z}_{2}$-graduado, isto é $L=L_{0} \oplus L_{1}$ que junto com uma aplicação bilinear $[\cdot, \cdot]: L \rightarrow L$ é tal que

1. $\left[L_{\alpha}, L_{\beta}\right] \subseteq L_{\alpha+\beta}, \quad \alpha, \beta \in \mathbb{Z}_{2}\left(\mathbb{Z}_{2}\right.$-graduação);

2. $[x, y]=-(-1)^{\alpha+\beta}[y, x], x \in L_{\alpha}, y \in L_{\beta}, \alpha, \beta \in \mathbb{Z}_{2}$, (anticomutatividade graduada);

3. $[x,[y, z]]=[[x, y], z]+(-1)^{\alpha+\beta}[y,[x, z]], x \in L_{\alpha}, y \in L_{\beta}$, (identidade de Jacobi graduada). 
Agora iremos definir a álgebra envelopante universal de uma superálgebra de Lie, e em seguida iremos enunciar a versão do Teorema de Poincaré-Birkhoff-Witt para estas álgebras.

Definição 1.3.30 Seja L uma superálgebra de Lie sobre um corpo K. Uma álgebra envelopante universal de $L$ é um par $(U(L), i)$, onde $U(L)$ é uma álgebra associativa com unidade sobre o corpo $K$ e $i: L \rightarrow U(L)$ é uma aplicação linear satisfazendo a seguinte relação

$$
i([x, y])=i(x) i(y)-(-1)^{\alpha+\beta} i(y) i(x),
$$

tal que a seguinte propriedade universal é satisfeita: se A é outra álgebra associativa com unidade sobre $K$ e $\varphi: L \rightarrow A$ é qualquer aplicação linear satisfazendo (1.7), então existe um único homomorfismo $\bar{\varphi}: U(L) \rightarrow$ A tal que $\bar{\varphi} \circ i=\varphi$.

Observe que a álgebra $A$ não necessariamente é $\mathbb{Z}_{2}$-graduada. Como $(U(L), i)$ é definida pela propriedade universal, a unicidade é determinada pelo argumento padrão.

Exibiremos agora a existência de $(U(L), i)$. Considere $T(L)$ a álgebra tensorial de $L$ e seja $I$ o ideal de $T(L)$ gerado por todos os elementos da forma

$$
x \otimes y-(-1)^{\alpha+\beta} y \otimes x-[x, y] .
$$

Então definimos $U(L)=T(L) / I$. A aplicação $i$ é a composição da inclusão $L \rightarrow T(L)$ e aplicação canônica $T(L) \rightarrow U(L)$. Note que $T(L)$ tem uma $\mathbb{Z}_{2}$-graduação que vem de $L$. Como o ideal $I$ é gerado por elementos homogêneos, segue que $U(L)$ é $\mathbb{Z}_{2}$-graduada também. Observamos também que $U(L)$ é gerada pela imagem de $i$ uma vez que $T(L)$ é gerada por $L$.

Agora, enunciaremos o Teorema de Poincaré-Birkhoff-Witt para superálgebras de Lie.

Teorema 1.3.31 Seja $L=L_{0} \oplus L_{1}$ uma superálgebra de Lie sobre um corpo $K$. Se $X_{0}=\left\{e_{i} \mid i \in I\right\}$ é uma base ordenada para $L_{0}$ e se $X_{1}=\left\{f_{j} \mid j \in J\right\}$ é uma base ordenada para $L_{1}$, então álgebra envelopante universal $U(L)$ de $L$ é gerada, como espaço, por todos os monômios do tipo

$$
e_{i_{1}}^{\alpha_{1}} e_{i_{2}}^{\alpha_{2}} \cdots e_{i_{n}}^{\alpha_{n}} f_{j_{1}}^{\beta_{1}} f_{j_{2}}^{\beta_{2}} \cdots f_{i_{m}}^{\beta_{m}}
$$

onde $i_{1}<i_{2}<\cdots<i_{n}, j_{1}<j_{2}<\cdots<j_{m}, \alpha_{i} \geq 0$ e $\beta_{j} \in\{0,1\}$. 
Em particular, se $L=L_{0} \oplus L_{1}$ é uma superálgebra de Lie abeliana onde $L_{0}=$ $\left\langle e_{1}, \ldots, e_{n}\right\rangle_{K}$ e $L_{1}=\left\langle f_{1}, \ldots, f_{m}\right\rangle_{K}$, então $U(L)$ é isomorfa ao produto tensorial (como espaço vetorial), das seguintes álgebras

$$
U(L) \cong K\left[x_{1}, \ldots, x_{n}\right] \otimes_{K} \Lambda\left(\xi_{1}, \ldots, \xi_{m}\right)
$$

onde $\Lambda\left(\xi_{1}, \ldots, \xi_{m}\right)=\left\langle\xi_{1}^{\alpha_{1}} \ldots \xi_{m}^{\alpha_{m}} \mid \alpha_{i} \in\{0,1\}\right\rangle_{K}$ é álgebra de Grassmann finitamente gerada.

\subsection{Grupos}

Nesta seção, iremos recordar algumas definições de grupos para que possamos mencionar os resultados contidos no próximo capítulo.

Definição 1.4.1 Um grupo é um par $(G, \cdot)$, onde $G$ é um conjunto não vazio e e é uma operação binária definida em $G$ satisfazendo os seguintes axiomas:

1. $(a \cdot b) \cdot c=a \cdot(b \cdot c)$, para todos $a, b, c \in G$, i.e., $\cdot$ é associativa;

2. existe um elemento $e_{G} \in G$, chamado identidade, tal que para todo a $\in G$, tem-se $e_{G} \cdot a=a \cdot e_{G}=a$

3. para cada $a \in G$ existe um elemento $a^{-1} \in G$, chamado inverso de a, tal que $a \cdot a^{-1}=a^{-1} \cdot a=e_{G}$.

O grupo $(G, \cdot)$ é chamado abeliano se $a \cdot b=b \cdot a$, para todos $a, b \in G$.

Definição 1.4.2 Seja p um primo e seja $G$ um grupo finito. Dizemos que $G$ é um p-grupo se a ordem de $G,|G|$, é um potência de $p$, isto é, se $|G|=p^{n}$, para algum $n$ inteiro não negativo.

Recorde que num grupo $G$, o produto comutador de dois elementos $g, h \in G$ é definido por

$$
[g, h]=g^{-1} h^{-1} g h
$$


O subgrupo $G^{\prime}$ é definido por

$$
G^{\prime}=[G, G]=\langle[g, h] \mid g, h \in G\rangle
$$

e é chamado de subgrupo comutador, ou ainda de subgrupo derivado de $G$.

Dizemos que o grupo $G$ é um grupo p-abeliano se o seu subgrupo comutador $G^{\prime}$ é um $p$-grupo. Claro que se $p=0$, então $G$ é abeliano.

Para qualquer grupo (finito ou infinito), definem-se os seguintes subgrupos indutivamente

$$
\gamma_{1}(G)=G, \quad \gamma_{2}(G)=\left[\gamma_{1}(G), G\right] \quad \text { e } \quad \gamma_{i+1}(G)=\left[\gamma_{i}(G), G\right]
$$

A cadeia de subgrupos

$$
\gamma_{1}(G) \geq \gamma_{2}(G) \geq \gamma_{3}(G) \geq \cdots
$$

é chamada a série central inferior de $G$.

O termo "inferior" indica que $\gamma_{i}(G) \geq \gamma_{i+1}(G)$.

Dizemos que o grupo $G$ é nilpotente quando $\gamma_{n}(G)=\left\{e_{G}\right\}$ para algum $n>0$. Se o interio $c+1 \geq 0$ é o menor com essa propriedade, dizemos que $G$ é nilpotente de classe $c$.

Agora, seja $K$ um anel (não necessariamente um corpo). Então, temos a seguir, a definição que combina um grupo $G$ com o anel $K$ que é o anel de grupo.

Definição 1.4.3 Seja K um anel comutativo com unidade e seja $G$ um grupo qualquer com a operação de grupo multiplicativa. Define-se o anel de grupo $K[G]$ do grupo $G$ como o $K$-módulo livre com base em $\{g \mid g \in G\}$ e coeficientes em $K$ como sendo o conjunto de todas as somas

$$
\sum_{g \in G} \alpha_{g} g, \quad \alpha_{g} \in K
$$

onde a multiplicação em $K[G]$ é dada por

$$
\left(\sum_{g \in G} \alpha_{g} g\right)\left(\sum_{h \in G} \beta_{h} h\right)=\sum_{g, h \in G} \alpha_{g} \beta_{h} g h, \quad \alpha_{g}, \beta_{h} \in K .
$$

Lema 1.4.4 Seja $K$ um anel comutativo e seja $G$ um grupo. Definimos a aplicação 
$f: K[G] \rightarrow K$ por

$$
f\left(\sum_{g \in G} \alpha_{g} g\right)=\sum_{g \in G} \alpha_{g}, \quad \alpha_{g} \in K
$$

Então $f$ é um homomorfimo de anéis sobrejetivo e $B=\left\{g-e_{G}: g \in G, g \neq e_{G}\right\}$ é uma base para o $K$-módulo livre ker $f$.

Demonstração. Obviamente, $f$ está bem definida, é aditiva e sobrejetiva. Agora, sejam $x=\sum_{g \in G} \alpha_{g} g$ e $y=\sum_{h \in G} \beta_{h} h$, então

$$
\begin{aligned}
f(x y) & =f\left(\sum_{g \in G}\left(\sum_{g_{1}, g_{2}=g} \alpha_{g_{1}} \beta_{g_{2}}\right) g\right) \\
& =\sum_{g \in G} \sum_{g_{1} g_{2}=g} \alpha_{g_{1}} \beta_{g_{2}} \\
& =\left(\sum_{g \in G} \alpha_{g}\right)\left(\sum_{g \in G} \beta_{g}\right) .
\end{aligned}
$$

Assim, $f$ é um homomorfismo de anéis. Logo, ker $f$ é um ideal de $K[G]$ e portanto um $K$-módulo. Agora,

$$
\begin{aligned}
& x=\left(\sum_{g \in G} \alpha_{g} g\right) \in \operatorname{ker} f \Leftrightarrow\left(\sum_{g \in G} \alpha_{g}\right)=0 \\
& \Leftrightarrow x=x-\left(\sum_{g \in G} \alpha_{g}\right) e_{G}=\sum_{g \in G} \alpha_{g}\left(g-e_{g}\right) .
\end{aligned}
$$

Portanto, $\operatorname{ker} f$, como um $K$-módulo, é gerado pelo conjunto $B=\left\{g-e_{G} \mid g \in G, g \neq\right.$ $\left.e_{G}\right\}$. Então, o conjunto $B$ ainda gera ker $f$. Para mostrar que $B$ é uma base para ker $f$ como um $K$-módulo suponhamos que $\sum_{g \in G} \alpha_{g}\left(g-e_{G}\right)=0$. Então,

$$
\sum_{g \in G} \alpha_{g} g=\left(\sum_{g \in G} \alpha_{g}\right) e_{G}
$$

Mas $g \neq e_{G}$ para todo $g \in B$ e então $\alpha_{g}=0$ para todo $g \in B$.

Definição 1.4.5 O homomorfismo de anéis $f$, como definido no lema acima, é chamado aplicação de aumento e o núcleo ker $f$ é chamado ideal de aumento de $K[G]$. 
Recorde que a ação de um grupo $G$ em um conjunto $X$ é um homomorfismo de $G$ no grupo das funções biunívocas de $X$, que é denotado por $B(X)$.

Denotando uma ação (à esquerda) por

$$
\begin{aligned}
\varphi: G & \rightarrow B(X) \\
g & \mapsto \varphi_{g}=\varphi(g)
\end{aligned}
$$

temos que $\varphi_{g}=\varphi(g)$ é uma bijeção no conjunto $X$, que associa a cada elemento $x \in X$ a um outro elemento $\varphi_{g}(x)$. Como $\varphi$ é um homomorfismo, então temos que

1. $\varphi_{g}\left(\varphi_{h}(x)\right)=\varphi_{g h}(x)$, para todos $g, h \in G$ e $x \in X$;

2. $\varphi_{e_{G}}=I d_{X}$, ou seja, $\varphi_{e_{G}}(x)=x$, para todo $x \in X$;

3. $\varphi_{g}^{-1}=\varphi_{g^{-1}}$, para todo $g \in G$.

Quando temos um grupo $G$ agindo sobre um grupo $H$ por automorfismos, nós construímos um novo grupo, isto é, munimos o produto cartesiano $H \times G$ com a seguinte operação

$$
\left(h_{1}, g_{1}\right) \cdot\left(h_{2}, g_{2}\right)=\left(h_{1} \varphi_{g_{1}}\left(h_{2}\right), g_{1} g_{2}\right)
$$

E com essa operação, o produto cartesiano ganha uma estrutura de grupo, que é o produto semidireto de $H$ por $G$ e é denotado por $H \rtimes_{\varphi} G$.

Seja $A$ uma álgebra sobre um corpo $K$. Sabemos que o conjunto dos automorfismos de $A, \operatorname{Aut}(A)$ é um grupo com respeito a composição de funções. Suponha que $G$ age na álgebra $A$ por automorfismos:

$$
\begin{aligned}
& \varphi: G \rightarrow \operatorname{Aut}(A) \\
& g \mapsto \varphi_{g}: A \rightarrow A \text {, } \\
& x \mapsto \varphi_{g}(x)
\end{aligned}
$$

$g \in G, x \in A$. Colocando $g * x=\varphi_{g}(x)=\varphi(g)(x)$, pode-se formar um novo espaço que codifica em si a estrutura do grupo $G$, a estrutura de $A$ e a ação $\varphi \operatorname{de} G \operatorname{em~} A$, o produto smash $R=A \# K[G]$. Isto é o espaço vetorial $R=A \otimes_{K} K[G]$ dotado com a multiplicação

$$
\left(a_{1}, g_{1}\right) \cdot\left(a_{2}, g_{2}\right)=\left(a_{1}\left(g_{1} * a_{2}\right), g_{1} g_{2}\right), \quad a_{1}, a_{2} \in A, g_{1}, g_{2} \in G
$$


Por linearidade, também o anel de grupo $K[G]$ age sobre $A$ :

$\left(\alpha_{1} g_{1}+\alpha_{2} g_{2}+\cdots+\alpha_{n} g_{n}\right) * a=\alpha_{1}\left(g_{1} * a\right)+\alpha_{2}\left(g_{2} * a\right)+\cdots+\alpha_{n}\left(g_{n} * a\right), \quad a \in A, g_{i} \in G, \alpha_{i} \in K$

Em particular, se um grupo $G$ age sobre uma álgebra de Lie $L$ (restrita), então essa ação pode ser estendida até a ação sobre a álgebra envelopante universal (restrita) $U(L)$ $(u(L))$ de $L$ e podemos formar o produto smash $U(L) \# K[G],(u(L) \# K[G])$. Para estes produtos, foram descobertos vários resultados com respeito as identidades polinomiais não triviais satisfeitos por eles. Mencionarems alguns deles no Capítulo 2.

\section{5 Álgebras de Poisson. Exemplos}

Álgebras de Poisson surgem naturalmente em diferentes áreas da álgebra, topologia e física.

Álgebras de Poisson foram introduzidas em 1976 por Berezin [7], veja também Vergne [55] (1969). Álgebras de Poisson foram usados para estudar álgebras envelopantes universais de álgebras de Lie, para álgebras de Lie de dimensão finita em característica zero [22], [32]. Subálgebras comutativas em álgebras de Poisson simétricas são usadas para estudar subálgebras comutativas em álgebras envelopantes universais de álgebras de Lie semissimples de dimensão finita em característica zero [52], [56].

A partir de agora, iremos considerar $K$ um corpo de caracterítica $p>0$, a menos dos casos específicos.

Um espaço vetorial $A$ sobre um corpo $K$ é uma álgebra de Poisson desde que, além de uma adição, $A$ tem duas operações $K$-bilineares as quais são relacionadas pela regra de Leibnitz. Mais precisamente, temos a seguinte definição.

Definição 1.5.1 Um K-espaço vetorial A é uma álgebra de Poisson se ele tem dois Kprodutos tais que:

1. A é uma álgebra associativa comutativa com unidade com multiplicação denotada por $a \cdot b$ (ou $a b)$, onde $a, b \in A$; 
2. A é uma álgebra de Lie em que tradicionalmente a operação de Lie é denotada pelos colchetes de Poisson $\{a, b\}$, onde $a, b \in A$;

3. estas duas operações estão relacionadas pela regra de Leibnitz:

$$
\{a \cdot b, c\}=a \cdot\{b, c\}+b \cdot\{a, c\}, \quad a, b, c \in A \text {. }
$$

Os exemplos típicos são dados a seguir.

Exemplo 1.5.2 Considere o anel de polinômios

$$
\mathbf{H}_{2 m}(K)=K\left[X_{1}, \ldots, X_{m}, Y_{1}, \ldots, Y_{m}\right]
$$

Se colocarmos $\left\{X_{i}, Y_{j}\right\}=\delta_{i j}$, onde $\delta_{i j}$ é o símbolo de Kronecker, e estendermos estes colchetes pela regra de derivação, a álgebra resultante é uma álgebra de Poisson com respeito a multiplicação natural, onde os colchetes de Poisson são

$$
\{f, g\}=\sum_{i=1}^{m}\left(\frac{\partial f}{\partial X_{i}} \frac{\partial g}{\partial Y_{i}}-\frac{\partial f}{\partial Y_{i}} \frac{\partial g}{\partial X_{i}}\right), \quad f, g \in \mathbf{H}_{2 m}(K)
$$

Nos referimos também à álgebra de Poisson $\mathbf{H}_{2 m}(K)$ como a álgebra Hamiltoniana.

Exemplo 1.5.3 Seja L uma álgebra de Lie sobre um corpo $K$, com $K$-base $\left\{v_{1}, v_{2}, \ldots\right\}$. Considere a álgebra simétrica da álgebra envelopante universal (veja [10], página 76):

$$
S(L)=\operatorname{gr}(U(L))=\bigoplus_{n=1}^{\infty} U_{n} / U_{n-1} \cong K\left[v_{1}, v_{2}, \ldots\right]
$$

um anel de polinômios. Definimos o colchete de Poisson por:

$$
\{v, w\}:=[v, w], \quad v, w \in L
$$

e estendendo por linearidade e por derivações:

$$
\left\{v_{i} \cdot v_{j}, v_{k}\right\}=v_{i} \cdot\left\{v_{j}, v_{k}\right\}+v_{j} \cdot\left\{v_{i}, v_{k}\right\}
$$

Assim, $S(L)$ é uma álgebra de Poisson, chamada álgebra de Poisson simétrica. 
Exemplo 1.5.4 Seja L uma álgebra de Lie sobre um corpo $K$ de característica $p>0$, com uma $K$-base $\left\{v_{1}, v_{2}, \ldots\right\}$. Considere uma álgebra quociente da álgebra de Poisson simétrica

$$
\mathbf{s}(L)=S(L) /\left(v^{p} \mid v \in L\right)
$$

Temos um anel de polinômios truncados. Por

$$
\left\{v^{p}, u\right\}=p v^{p-1}\{v, u\}=0, \quad v \in L, u \in \mathbf{s}(L)
$$

os colchetes de Poisson sobre $S(L)$ produzem um colchete de Poisson sobre $\mathbf{s}(L)$. Assim, $\mathbf{s}(L)$ é uma álgebra de Poisson chamada álgebra simétrica truncada. Observe que L não necessariamente é uma álgebra de Lie restrita.

Exemplo 1.5.5 Seja K um corpo da caraterística positiva $p$. Introduzimos a álgebra de Poisson Hamiltoniana truncada como

$$
\mathbf{h}_{2 m}(K)=K\left[X_{1}, \ldots, X_{m}, Y_{1}, \ldots, Y_{m}\right] /\left(X_{i}^{p}, Y_{i}^{p} \mid i=1, \ldots, m\right)
$$

onde o colchete é definido por (1.8), veja Exemplo 1.5.2, e usando a observação do Exemplo 1.5.3.

As álgebras Hamiltonianas $\mathbf{h}_{2 m}(K)$ e $\mathbf{H}_{2 m}(K)$ na classe das álgebras de Poisson tem um papel semelhante ao das álgebras de matrizes $\mathrm{M}_{n}(K)$ na classe das álgebras associativas.

As noções de subálgebra de Poisson, ideais de Poisson e morfismos de Poisson, são conceitos padrões e são definidos em termos dos colchetes de Poisson.

Definição 1.5.6 Seja A uma álgebra de Poisson sobre um corpo $K$ e sejam $H$ e I subespaços de A. Então

1. H é dita subálgebra de Poisson se $H \cdot H \subseteq H$ e $\{H, H\} \subseteq H$;

2. I é dito ideal de Poisson se $I \cdot A \subseteq I$ e $\{I, A\} \subseteq I$. 
Definição 1.5.7 Sejam duas álgebras de Poisson $A_{1}$ e $A_{2}$ sobre um corpo $K$. Um homomorfismo de álgebras $\varphi: A_{1} \rightarrow A_{2}$ é chamado homomorfismo de álgebras de Poisson se $\varphi$ preserva os colchetes de Poisson, isto é, para quaisquer $f, g \in A_{1}$,

$$
\varphi\{f, g\}_{A_{1}}=\{\varphi(f), \varphi(g)\}_{A_{2}} .
$$

\section{6 Álgebra de Poisson livre e identidades de Poisson}

O objetivo desta seção é descrever alguns fatos básicos sobre identidades polinomiais de álgebras de Poisson.

Seja $K$ um corpo qualquer de característica 0 e seja $X=\left\{x_{1}, x_{2}, \ldots\right\}$ um conjunto não vazio de variáveis enumerável.

Definição 1.6.1 A álgebra de Poisson livre sobre $X$ é uma álgebra de Poisson $P(X)$ que junto com uma aplicação $i: X \rightarrow P(X)$ é tal que dada qualquer aplicação $\varphi: X \rightarrow A$ em uma álgebra de Poisson $A$, ela pode ser unicamente estendida a um homomorfismo de álgebras de Poisson $\bar{\varphi}: P(X) \rightarrow$ A tal que o diagrama

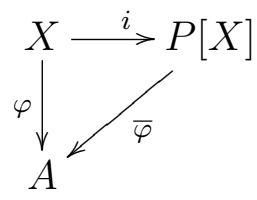

é comutativo, isto é, $\varphi=\bar{\varphi} \circ i$.

Considere a álgebra de Lie livre $L=L(X)$ sobre o conjunto das variáveis $X$, e sua álgebra simétrica $F(X)=S(L(X))$, como na Definição 1.3.22. Então, $F(X)$ é a álgebra de Poisson livre nas variáveis $x \in X$, como foi demonstrado por Shestakov [44].

Por exemplo, seja $L=L(x, y)$ a álgebra de Lie livre de posto 2. Consideramos a base de Hall (veja Teorema 1.3.10) dela

$$
L=\langle x, y,[y, x],[[y, x], x],[[y, x], y],[[[y, x], x], x], \ldots\rangle_{K}
$$


Obtemos a álgebra de Poisson livre $S(L)$ de posto 2, que tem uma base canônica nos monômios como a abaixo:

$$
\left\langle x^{n_{1}} y^{n_{2}}\{y, x\}^{n_{3}}\{\{y, x\}, x\}^{n_{4}}\{\{y, x\}, y\}^{n_{5}}\{\{\{y, x\}, x\}, x\}^{n_{6}} \ldots \mid n_{i} \geq 0\right\rangle_{K},
$$

onde apenas uma quantidade finita de $n_{i}$ 's, $i \geq 1$, são diferentes de zero.

A definição de uma PI-álgebra de Poisson é padrão, as identidades sendo elementos da álgebra de Poisson livre $F(X)$ de posto enumerável. Assumiremos que os fatos básicos sobre variedades de álgebras lineares são conhecidas do leitor, (veja, por exemplo, [11] e [1]).

Considere a álgebra de Poisson livre $F(X)$ com conjunto gerador enumerável $X=$ $\left\{x_{i} \mid i \in \mathbb{N}\right\}$. Seja $P_{n}=P_{n}(X) \subset F(X)$ o subespaço dos elementos multilineares de grau $n$ em $x_{1}, \ldots, x_{n}$. Considere também o subespaço $Q_{2 n} \subset P_{2 n}$ gerado pelos elementos

$$
\left\{x_{\alpha_{1}}, x_{\alpha_{2}}\right\} \cdot\left\{x_{\alpha_{3}}, x_{\alpha_{4}}\right\} \cdots\left\{x_{\alpha_{2 n-1}}, x_{\alpha_{2 n}}\right\}
$$

Segundo Farkas [13], chamamos os elementos de $Q_{2 n}$ de polinômios customary. Uma base canônica de $Q_{2 n}$ é dada a seguir

$$
\begin{gathered}
Q_{2 n}=\left\langle\left\{x_{\tau(1)}, x_{\tau(2)}\right\} \cdot\left\{x_{\tau(3)}, x_{\tau(4)}\right\} \cdots\left\{x_{\tau(2 n-1)}, x_{\tau(2 n)}\right\}\right| \tau \in S_{2 n}, \\
\tau(1)<\tau(2), \tau(3)<\tau(4), \ldots, \tau(2 n-1)<\tau(2 n), \\
\tau(1)<\tau(3)<\cdots<\tau(2 n-1)\rangle_{K} .
\end{gathered}
$$

Denote por $T_{2 n}$ o conjunto das permutações $\tau \in S_{2 n}$ satisfazendo as condições acima. Note que $\left|T_{2 n}\right|$ é igual ao número de partições do conjunto $\{1,2, \ldots, 2 n\}$ em pares.

Escrevemos uma identidade customary não trivial como:

$$
\sum_{\tau \in T_{2 n}} \lambda_{\tau}\left\{x_{\tau(1)}, x_{\tau(2)}\right\} \cdot\left\{x_{\tau(3)}, x_{\tau(4)}\right\} \cdots\left\{x_{\tau(2 n-1)}, x_{\tau(2 n)}\right\} \equiv 0, \quad \lambda_{\tau} \in K
$$

onde $\lambda_{e}=1$, para a permutação identidade $e \in T_{2 n}$.

A importância de polinômios customary é explicado pelo seguinte fato descoberto por Farkas. 
Teorema 1.6.2 (Farkas, 1998, [13]) Suponha que $\mathcal{V}$ é uma variedade álgebras de Poisson não trivial sobre um corpo $K$ de característica zero. Então $\mathcal{V}$ satisfaz uma identidade customary não trivial.

Vamos mostrar a ideia da demonstração. Seja uma álgebra de Poisson $R$ que satisfaz a identidade $f(X, Y, Z)=\{\{X, Y\}, Z\} \equiv 0$. Então, $R$ satisfaz também a identidade:

$$
\begin{aligned}
0 & \equiv f\left(X_{1} X_{2}, Y, Z\right)-X_{1} f\left(X_{2}, Y, Z\right)-X_{2} f\left(X_{1}, Y, Z\right) \\
& =\left\{\left\{X_{1} X_{2}, Y\right\}, Z\right\}-X_{1}\left\{\left\{X_{2}, Y\right\}, Z\right\}-X_{2}\left\{\left\{X_{1}, Y\right\}, Z\right\} \\
& =\left\{X_{1}, Y\right\}\left\{X_{2}, Z\right\}+\left\{X_{2}, Y\right\}\left\{X_{1}, Z\right\},
\end{aligned}
$$

obtemos um polinômio customary. Farkas chamou isso de processo de customarização [13], que é um análogo do processo de linearização para álgebras associativas bem conhecido.

De fato, os argumentos de [13], provam o seguinte resultado.

Teorema 1.6.3 (Farkas, 1998, [13]) Suponha que uma álgebra de Poisson A satisfaz uma identidade de Poisson multilinear não trivial. Então A satisfaz uma identidade customary não trivial.

Vamos explicar porque nós precisamos de polinômios multilineares no caso da característica positiva $p$. O processo de linearização simplesmente não funciona para álgebras de Poisson em característica positiva como no caso de álgebras associativas ou de Lie. De fato, por exemplo, a identidade de Poisson $\{x, y\}^{p} \equiv 0$ é dada por um elemento não nulo da álgebra de Poisson livre $F(X)$. Observe que sua linearização completa é trivial:

$$
\sum_{\sigma, \pi \in \mathbf{s}}\left\{x_{\sigma(1)}, y_{\pi(1)}\right\} \cdots\left\{x_{\sigma(p)}, y_{\pi(p)}\right\}=p ! \sum_{\pi \in \mathbf{s}}\left\{x_{1}, y_{\pi(1)}\right\} \cdots\left\{x_{p}, y_{\pi(p)}\right\}=0
$$

Além disso, checa-se que qualquer álgebra simétrica truncada $\mathbf{s}(L)$ satisfaz a identidade $\{x, y\}^{p} \equiv 0$. Com efeito, sejam $a, b \in \mathbf{s}(L)$, então $\{a, b\}$ é um polinômio truncado sem o termo constante, sua $p$ - ésima potência é zero pela regra de Frobenius $(v+w)^{p}=v^{p}+w^{p}$. Assim, não faz sentido estudar identidades de Poisson arbitrárias para álgebras simétricas truncadas.

Na teoria de PI-álgebras de Poisson, o análogo do polinômio standard associativo é o 
polinômio de Poisson standard como abaixo ([13], [14]):

$$
\mathrm{St}_{2 n}=\mathrm{St}_{2 n}\left(x_{1}, \ldots, x_{2 n}\right)=\sum_{\sigma \in T_{2 n}}(-1)^{\sigma}\left\{x_{\sigma(1)}, x_{\sigma(2)}\right\} \cdots\left\{x_{\sigma(2 n-1)}, x_{\sigma(2 n)}\right\}
$$

Este é um polinômio customary e ele é antissimétrico em todas as variáveis [13]. Temos o seguinte fato semelhante ao de álgebras associativas.

Teorema 1.6.4 (Mishchenko, Petrogradsky, Regev, 2007, [29]) No caso de característica zero, qualquer PI-álgebra de Poisson satisfaz uma identidade

$$
\left(\operatorname{St}_{2 n}\left(x_{1}, \ldots, x_{2 n}\right)\right)^{m} \equiv 0
$$

para alguns inteiros $n, m$.

Outro fato importante sobre identidades standard é como a seguir.

Lema 1.6.5 (Farkas, 1998, [13]) Seja A uma álgebra de Poisson sobre um corpo arbitrário $K$ gerada por $k$ elementos como uma álgebra associativa. Então A satisfaz a identidade standard

$$
\operatorname{St}_{2 m}\left(x_{1}, \ldots, x_{2 m}\right) \equiv 0
$$

sempre que $2 m>k$. 


\section{Capítulo 2}

\section{Identidades dos anéis de grupos e álgebras envelopantes}

Neste capítulo, nós fazemos uma breve exposição dos resultados sobre a existência de identidades polinomiais não triviais dos anéis de grupos, álgebras envelopantes, produtos smash e álgebras de Poisson.

\subsection{Identidades dos anéis de grupos}

Passman obteve condições necessárias e suficientes para o anel de grupo $K[G]$ satisfazer uma identidade polinomial não trivial sobre um corpo $K$ de característica qualquer.

Teorema 2.1.1 (Passman, 1972, [34]) A álgebra de grupo $K[G]$ de um grupo $G$ satisfaz uma identidade polinomial não trivial se, e somente se, as seguintes condições são satisfeitas.

1. Existe um subgrupo normal $A \subset G$ de indice finito;

2. A é abeliano se $K$ é de característica zero, e o subgrupo comutador $A^{\prime}$ é um p-grupo finito se $K$ é de característica $p>0$. 
Recordando que um grupo $G$ é dito $4 p$-abeliano se $G$ é abeliano para $p=0$ e se $p>0$, $G^{\prime}$, o subgrupo comutador de $G$, é um $p$-grupo finito.

Em [33] Passi, Passman e Sehgal consideraram identidades polinomiais que correspondem a Lie nilpotência e a Lie solubilidade de $K[G]$.

Teorema 2.1.2 (Passi, Passman, Sehgal, 1973, [33]) Seja $K[G]$ um anel de grupo de um grupo $G$ sobre um corpo $K$, char $K=p \geq 0$. Então

1. $K[G]$ é Lie nilpotente se, e somente se, $G$ é p-abeliano e nilpotente;

2. $K[G]$ é Lie solúvel se, e somente se,

(i) G é p-abeliano, para $p \neq 2$ e

(ii) $G$ tem um subgrupo 2-abeliano de índice no máximo 2, para $p=2$.

Existe uma fórmula para a classe de Lie nilpotência de um anel de grupo sobre um corpo de característica positiva. Seja $G$ um grupo, $K[G]$ o anel de grupo do grupo $G$, define-se os subgrupos de dimensão de Lie de $G$ como:

$$
D_{(n), K}(G)=G \cap\left(1+K[G]^{(n)}\right), \quad n \geq 0,
$$

onde $K[G]^{(n)}$ é o $n$-ésimo termo das potências de Lie superiores de $K[G]$.

Bhandari e Passi descreveram os subgrupos de dimensão de Lie como [9]:

$$
D_{(n), K}(G)=\prod_{(i-1) p^{k} \geq n} \gamma_{i}(G)^{p^{k}}, \quad n \geq 0 .
$$

Teorema 2.1.3 (Bhandari, Passi, 1992, [9]) Sejam $G$ um grupo, $K$ um corpo de característica $p>3$ tais que o anel de grupo $K[G]$ é Lie nilpotente. Então a classe de Lie nilpotência de $K[G]$ é igual a

$$
1+(p-1) \sum_{m \geq 1} m \log _{p}\left(D_{(m), K}(G): D_{(m+1), K}(G)\right)
$$




\subsection{Identidades das álgebras envelopantes universais}

Em [26], Latyshev provou que a álgebra envelopante universal de uma álgebra de Lie de dimensão finita sobre um corpo de caraterística zero satisfaz uma identidade não trivial se, e somente se, a álgebra de Lie é abeliana. Mais tarde, Bahturin notou que a condição de a álgebra de Lie ser de dimensão finita não era necessária, (veja [1]).

Bahturin estabeleceu também um teorema semelhante sobre a existência de uma identidade não trivial para a álgebra envelopante sobre um corpo de característica positiva como a seguir.

Teorema 2.2.1 (Bahturin, 1974, [2]) Seja L uma álgebra de Lie sobre um corpo $K$ de característica positiva. Então a álgebra envelopante universal $U(L)$ satisfaz a uma identidade polinomial não trivial se, e somente se, as seguintes condições são satisfeitas:

1. existe um ideal abeliano $H \subset L$ de codimensão finita;

2. todas as derivações internas ad $x, x \in L$ são algébricas de grau limitado.

Além disso, Bahturin encontrou condições necessárias e suficientes para a álgebra envelopante universal de uma superálgebra de Lie sobre um corpo de característica zero satisfazer uma identidade polinomial não trivial [3].

Teorema 2.2.2 (Bahturin, 1985, [3]) Sejam K um corpo de característica zero, $L=$ $L_{0} \oplus L_{1}$ uma superálgebra de Lie e $U(L)$ sua álgebra envelopante universal. Então $U(L)$ satisfaz a uma identidade polinomial não trivial se, e somente se, as seguintes condições são válidas:

1. L L é uma álgebra de Lie abeliana;

2. $L_{1}$ contém um $L_{0}$-submódulo $M$ tal que ambos $L_{1} / M$ e $\left[L_{0}, M\right]$ são de dimensão finita;

3. $[M, M]=0$. 


\subsection{Identidades das álgebras envelopantes restritas}

Passman em [36] e Petrogradsky em [37] descreveram a álgebra de Lie restrita cuja álgebra envelopante restrita $u(L)$ satisfaz a uma identidade polinomial não trivial. Isso deu o resultado principal para nossa pesquisa.

Teorema 2.3.1 (Passman, 1990, [36], Petrogradsky, 1991, [37]) Seja L uma p-álgebra de Lie. Então a álgebra envelopante restrita $u(L)$ satisfaz a uma identidade polinomial não trivial se, e somente se, existem ideais restritos $Q \subset H \subset L$ tais que

1. $\operatorname{dim} L / H<\infty, \operatorname{dim} Q<\infty$;

2. $H / Q$ é abeliano;

3. Q é abeliano e tem uma p-aplicação nilpotente.

Em [42], Riley e Shalev determinaram quando $u(L)$, considerada como uma álgebra de Lie, é solúvel (para $p>2$ ), nilpotente e quando ela é $n$-Engel, isto é, quando ela satisfaz a identidade $[x, n]=[x, y, \ldots, y] \equiv 0$, onde $y$ aparece na expressão $n$ vezes.

Teorema 2.3.2 (Riley, Shalev, 1993, [42]) Seja u $(L)$ a álgebra envelopante restrita de uma álgebra de Lie restrita $L$ sobre um corpo $K$, de característica $p>0$. Então

1. $u(L)$ é Lie nilpotente se, e somente se, $L$ é nilpotente, $L^{2}$ é de dimensão finita e p-nilpotente;

2. $u(L)$ é n-Engel para algum $n$ se, e somente se, $L$ é nilpotente, $L^{2}$ é p-nilpotente e $L$ tem um ideal restrito $A$ tal que ambos $L / A$ e $A^{\prime}$ são de dimensão finita.

3. $u(L)$ é Lie solúvel se, e somente se, $L^{2}$ é de dimensão finita e p-nilpotente, para $p \neq 2$.

No Teorema 2.3.2, p-nilpotente denota que a $p$-aplicação é nil.

Seja $L$ uma álgebra de Lie restrita sobre um corpo $K$ de característica $p$, Riley e Shalev definiram as subálgebras de dimensão de Lie superiores de $L$ como abaixo [41]:

$$
D_{(n)}(L)=L \cap u(L)^{(n)}, \quad n \geq 0,
$$


onde $u(L)^{(n)}$ é o $n$-ésimo termo das potências de Lie superiores da álgebra $u(L)$.

(Nossa enumeração é alterada em compação com [41]). Riley e Shalev obtiveram outras descrições semelhantes aquelas (2.1) para anéis de grupo [41]:

$$
D_{(n)}(L)=\sum_{(i-1) p^{k} \geq n} \gamma_{i}(L)^{p^{k}}, \quad n \geq 0
$$

O seguinte é um resultado análogo para classes de Lie nilpotência para anéis de grupo (veja Teorema 2.1.3).

Teorema 2.3.3 (Riley, Shalev, 1995, [41]) Seja L uma álgebra de Lie restrita sobre um corpo $K$ de característica p tal que $u(L)$ é Lie nilpotente. Então

1. A classe de Lie nilpotência forte de $u(L)$ é igual a

$$
1+(p-1) \sum_{m \geq 1} m \operatorname{dim}\left(D_{(m)} / D_{(m+1)}\right)
$$

2. No caso $p>3$, a classe de Lie nilpotência coincide com a classe de Lie nilpotência forte.

O caso quando o corpo tem característica 2 foi determinado em [50].

Teorema 2.3.4 (Siciliano, Usefi, 2015, [50]) Seja L uma álgebra de Lie restrita sobre um corpo $K$ de característica 2 . Seja $\bar{K}$ o fecho algébrico de $K$ e coloque $\mathcal{L}=L \otimes_{K} \bar{K}$. Então $u(L)$ é Lie solúvel se, e somente se L tem um ideal I restrito 2-nilpotente de dimensão finita tal que $\overline{\mathcal{L}}=\mathcal{L} / I$ satisfaz uma das seguintes condições:

1. $\overline{\mathcal{L}}$ tem um ideal restrito abeliano de codimensão no máximo $1 ;$

2. $\overline{\mathcal{L}}$ é nilpotente de classe 2 e $\operatorname{dim} \overline{\mathcal{L}} / Z(\overline{\mathcal{L}})=3$;

3. $\overline{\mathcal{L}}=\left\langle x_{1}, x_{2}, y\right\rangle_{\bar{K}} \oplus Z(\overline{\mathcal{L}})$, onde $\left[x_{1}, y\right]=x_{1},\left[x_{2}, y\right]=x_{2}, e\left[x_{1}, x_{2}\right] \in Z(\overline{\mathcal{L}})$;

4. $\overline{\mathcal{L}}=\langle x, y\rangle_{\bar{K}} \oplus H \oplus Z(\overline{\mathcal{L}})$, onde $H$ é uma subálgebra restrita abeliana forte de dimensão finita de $\overline{\mathcal{L}}$ tal que $[x, y]=x,[y, h]=h, e[x, h] \in Z(\overline{\mathcal{L}})$ para cada $h \in H ;$ 
5. $\overline{\mathcal{L}}=\langle x, y\rangle_{\bar{K}} \oplus H \oplus Z(\overline{\mathcal{L}})$, onde $H$ é uma subálgebra abeliana de dimensão finita de $\overline{\mathcal{L}}$ tal que $[x, y]=x,[y, h]=h,[x, h] \in Z(\overline{\mathcal{L}}), e[x, h]^{[2]}=h^{[2]}$, para cada $h \in H$.

Siciliano provou em [46] que, no caso $p>2$, a solubilidade forte da álgebra envelopante restrita $u(L)$ é equivalente a sua solubilidade. Também, a solubilidade forte, no caso $p=2$, é descrita pela mesma condição dada na Parte 3 do Teorema 2.3.2. No caso $p=2$, ele mostrou um exemplo de uma álgebra envelopante restrita $u(L)$ que é solúvel mas não é solúvel forte.

Lie solubilidade, Lie nilpotência e outras identidades não matriciais para álgebras envelopantes (restritas) de superálgebras de Lie (restritas) são estudadas em [8], [48], [47], [53] e [54]. Mais sobre comprimentos solubilidade de Lie, classes de Lie nilpotência para $u(L)$, ou elementos simétricos de $u(L)$, etc, veja [49].

Para o caso de $p$-superálgebras de Lie, veja em [2], p-superálgebras de Lie coloridas, veja em [4].

\subsection{Identidades dos produtos smash}

Outros desenvolvimentos foram obtidos para o produto smash da forma $U(L) \# K[G]$ e $u(L) \# K[G]$ em [5], onde um grupo $G$ age por automorfismos sobre uma álgebra de Lie (restrita) L. Estes resultados são interessantes porque eles combinam ambas situações, anéis de grupo e álgebras envelopantes.

Teorema 2.4.1 (Bahturin, Petrogradsky, 2002, [5]) Suponha que um grupo G age por automorfismos sobre uma p-álgebra de Lie L. Então u $(L) \# K[G]$ é uma PI-álgebra se, e somente se,

1. existem subálgebras restritas $G$-invariantes $Q \subset H \subset L$ com

(a) $\operatorname{dim} L / H<\infty, \operatorname{dim} Q<\infty$;

(b) $[H, H] \subset Q$;

(c) Q é abeliana com uma p-aplicação nilpotente;

2. existe um subgrupo $A \subset G$ com 
(a) $|G: A|<\infty$;

(b) o subgrupo comutador $A^{\prime}$ é um p-grupo abeliano finito;

3. A age trivialmente sobre $H / Q$.

Teorema 2.4.2 (Bahturin, Petrogradsky, 2002, [5]) Sejam G um grupo, L uma álgebra de Lie sobre um corpo $K$ de característica $p>0$ e suponha que $G$ age sobre $L$ por automorfismos. Então $U(L) \# K[G]$ é PI-álgebra, se e somente se, as seguintes afirmações são válidas:

1. existe um ideal abeliano $G$-invariante $H \subset L$ de codimensão finita e todas as derivações internas ad $x$, para qualquer $x \in L$, são algébricos de grau limitado;

2. existe um subgrupo normal $A \subset G$ de indice finito tal que $A^{\prime}$, o subgrupo comutador, é um p-grupo finito abeliano;

3. A age trivialmente sobre $L$.

Identidades para o produto smash $U(L) \# K[G]$, onde $L$ é uma superálgebra de Lie em característica zero foram estudadas em [21].

Teorema 2.4.3 (Kochetov, 2003, [21]) Seja $L=L_{0} \oplus L_{1}$ uma superálgebra de Lie sobre um corpo $K$ de característica zero. Seja $G$ um grupo agindo sobre L por automorfismos de álgebra graduada. Então o produto smash $U(L) \# K[G]$ é PI-álgebra, se e somente se, existem subespaços $N$ e $M L_{0}$ - e G-invariantes com $N \subset M \subset L_{1}$ e um subgrupo $A$ de $G$ tais que:

1. $N$ e $L_{1} / M$ são de dimensão finita;

2. $\left[L_{0}, L_{0}\right]=[M, M]=0$ e $\left[L_{0}, M\right] \subset N$;

3. A é abeliano e tem indice finito em $G$;

4. A age trivialmente sobre $L_{0}$ e sobre $M / N$. 


\subsection{Identidades das álgebras de Poisson simétricas}

O seguinte resultado é um análogo do teorema clássico de Amitsur-Levitzki sobre identidades de anéis de matrizes. De fato, Kostant usou uma outra linguagem, mas como observou Farkas [14], Kostant na verdade demonstrou o resultado sobre identidades de álgebras de Poisson.

Teorema 2.5.1 (Kostant, 1981, [22]) Seja L uma álgebra de Lie de dimensão finita sobre um corpo de característica zero. Então, a álgebra de Poisson simétrica $S(L)$ satisfaz a identidade de Poisson standard $\mathrm{St}_{2 d} \equiv 0$ sempre que $2 d$ excede a dimensão da órbita coadjunta máxima de $L$.

A nilpotência de classe 2 das álgebras simétricas foi considerada por Shestakov.

Teorema 2.5.2 (Shestakov, 1993, [44]) A álgebra simétrica $S(L)$ de uma álgebra de Lie arbitrária $L$ satisfaz uma identidade de Poisson $\{x,\{y, z\}\} \equiv 0$ se, e somente se, $L$ é abeliana.

Em [14], Farkas provou a seguinte afirmação semelhante ao Teorema 2.5.1 de Kostant.

Teorema 2.5.3 (Farkas, 1999, [14]) Seja L uma álgebra de Lie sobre um corpo de característica zero. Então a álgebra simétrica $S(L)$ satisfaz a uma identidade de Poisson não trivial se, e somente se, L contém uma subálgebra abeliana de codimensão finita.

Giambruno e Petrogradsky em [15] estenderam este resultado para uma álgebra de característica arbitrária.

Teorema 2.5.4 (Giambruno, Petrogradsky, 2006, [15]) Seja L uma álgebra de Lie sobre um corpo de característica arbitrária. Então a álgebra simétrica $S(L)$ satisfaz uma identidade de Poisson multilinear não trivial se, e somente se, L contém uma subálgebra abeliana de codimensão finita.

O seguinte resultado foi obtido para a álgebra simétrica truncada $\mathbf{s}(L)$ de uma $p$ álgebra de Lie. 
Teorema 2.5.5 (Giambruno, Petrogradsky, 2006, [15]) Seja L uma p-álgebra de Lie. Então a álgebra simétrica truncada $\mathbf{s}(L)$ satisfaz uma identidade de Poisson multilinear não trivial se, e somente se, existe um ideal restrito $H \subset L$ tal que

1. $\operatorname{dim} L / H<\infty$,

2. $\operatorname{dim} H^{2}<\infty$;

3. $H$ é nilpotente de classe 2.

Este resultado é fundamental para nós porque as identidades de Lie nilpotência (forte) e solubilidade (forte) são multilineares e nós usamos ele na forma do Teorema 4.1.4, veja abaixo. 


\section{Capítulo 3}

\section{Resultados principais}

Neste capítulo, introduzimos duas noções de Lie nilpotência e Lie solubilidade para as álgebras de Poisson, definindo as séries centrais inferiores, potências de Lie superiores, séries derivadas e séries derivadas superiores. Depois, apresentamos nossos resultados principais. O primeiro nos dá condições equivalentes com respeito as identidades de Lie nilpotência e Lie nilpotência forte da álgebra de Poisson simétrica truncada $\mathbf{s}(L)$. O segundo nos dá condições equivalentes com respeito as identidades de Lie solúvel e Lie solúvel forte, neste caso em característica $p \geq 3$. Usaremos os termos Lie solúvel/Lie nilpotente quando nos referimos a estrutura de Lie de álgebras de Poisson.

\subsection{Duas noções de Lie nilpotência de álgebras de Poisson}

Seja $R$ uma álgebra de Poisson arbitrária. Para cada inteiro positivo $m$, denote por $\gamma_{m}(R)$ o $m$-ésimo termo da série central inferior de $R$, i.e., $\gamma_{1}(R)=R$ e $\gamma_{n+1}(R)=$ $\left\{\gamma_{n}(R), R\right\}, n \geq 1$. Isto nos dá uma sequência decrescente de ideais

$$
R=\gamma_{1}(R) \supseteq \gamma_{2}(R)=\{R, R\} \supseteq \gamma_{3}(R) \supseteq \cdots
$$

Considere também os seus ideais gerados $R^{[n]}=\gamma_{n}(R) R, n \geq 1$, chamados de 
potências de Lie inferiores. Dizemos que $R$ é Lie nilpotente de classe $s$ se $\gamma_{s+1}(R)=0$ mas $\gamma_{s}(R) \neq 0$. Claramente, a condição $\gamma_{s+1}(R)=0$ é equivalente a $R^{[s+1]}=0$ ou à identidade de Lie nilpotência:

$$
\left\{\ldots\left\{\left\{X_{0}, X_{1}\right\}, X_{2}\right\}, \ldots, X_{s}\right\} \equiv 0
$$

Definimos também as chamadas potências de Lie superiores como a seguir. A cada passo nós pegamos o ideal gerado pelos comutadores, isto é, colocamos $R^{(0)}=R$ e $R^{(n)}=$ $\left\{R^{(n-1)}, R\right\} R$ para todo $n \geq 1$, (observe que estamos usando a numeração alterada de potências de Lie superiores que foi feito em [41]). Também ganhamos uma sequência decrescente de ideais de $R$. A condição $R^{(d)} \equiv 0$ é equivalente a identidade de Lie nilpotência forte de classe $d$

$$
\left\{\left\{\ldots\left\{\left\{X_{0}, X_{1}\right\} \cdot Y_{1}, X_{2}\right\} \cdot Y_{2}, \ldots, X_{d-1}\right\} \cdot Y_{d-1}, X_{d}\right\} \equiv 0
$$

Assim, dizemos que uma álgebra de Poisson $R$ é Lie nilpotente forte de classe $d$ quando $R$ satisfaz a identidade (3.1), ou equivalentemente que $R^{(d+1)} \equiv 0$ e não satisfaz condição semelhante para $d$. Observe também que

$$
\gamma_{n}(R) \subseteq R^{(n-1)}, n \geq 1
$$

Isto significa que a Lie nilpotência forte de classe $d$ implica a Lie nilpotência de mesma classe $d$. A nilpotência de classe 1 é equivalente a nilpotência forte de classe 1 e é equivalente ao fato de $R$ ser uma álgebra de Lie abeliana.

\subsection{Duas noções de Lie solubilidade de álgebras de Poisson}

Seja $R$ uma álgebra de Poisson arbitrária. Para cada inteiro positivo $m$, denotaremos por $\delta_{m}(R)$ o $m$-ésimo termo da série derivada de $R$, i.e., $\delta_{0}(R)=R, \delta_{n+1}(R)=$ $\left\{\delta_{n}(R), \delta_{n}(R)\right\}, n \geq 0$. 
Por indução, definimos polinômios:

$$
\begin{aligned}
\delta_{1}\left(X_{1}, X_{2}\right)= & \left\{X_{1}, X_{2}\right\} ; \\
\delta_{2}\left(X_{1}, X_{2}, X_{3}, X_{4}\right)=\left\{\left\{X_{1}, X_{2}\right\},\left\{X_{3}, X_{4}\right\}\right\} ; & \vdots \\
\delta_{s+1}\left(X_{1}, X_{2}, \ldots, X_{2^{s+1}}\right)= & \left\{\delta_{s}\left(X_{1}, \ldots, X_{2^{s}}\right), \delta_{s}\left(X_{2^{s+1}+1}, \ldots, X_{2^{s+1}}\right)\right\} ;
\end{aligned}
$$

Agora, $R$ é dita Lie solúvel de classe $s$ se, e somente se, $\delta_{s}(R)=0$, mas $\delta_{s-1}(R) \neq 0$, ou equivalentemente, $R$ satisfaz a identidade de Lie solubilidade $\delta_{s}\left(X_{1}, \ldots, X_{2^{s}}\right) \equiv 0$ (onde $s$ é minimal). Uma outra condição equivalente é que existe a cadeia dos ideais de Lie

$$
R=R_{0} \supset R_{1} \supset \cdots \supset R_{s}=0
$$

tais que os fatores $R_{i} / R_{i+1}, i=0, \ldots, s-1$, são abelianos e $s$ é minimal.

Definimos também as chamadas séries derivadas superiores. Colocamos $\widetilde{\delta}_{0}(R)=R$, $\widetilde{\delta}_{n+1}(R)=\left\{\widetilde{\delta}_{n}(R), \widetilde{\delta}_{n}(R)\right\} \cdot R, n \geq 0$. Por indução, definimos polinômios como:

$$
\begin{gathered}
\widetilde{\delta}_{1}\left(X_{1}, X_{2}, Y_{1}\right)=\left\{X_{1}, X_{2}\right\} Y_{1} ; \\
\widetilde{\delta}_{2}\left(X_{1}, X_{2}, X_{3}, X_{4}, Y_{1}, Y_{2}, Y_{3}\right)=\left\{\left\{X_{1}, X_{2}\right\} Y_{1},\left\{X_{3}, X_{4}\right\} Y_{2}\right\} Y_{3} ; \\
\vdots \\
\widetilde{\delta}_{n+1}\left(X_{1}, \ldots, X_{2^{n+1}}, Y_{1}, \ldots, Y_{2^{n+1}-1}\right)= \\
\left\{\widetilde{\delta}_{n}\left(X_{1}, \ldots, X_{2^{n}}, Y_{1}, \ldots, Y_{2^{n}-1}\right),\right. \\
\left.\widetilde{\delta}_{n}\left(X_{2^{n}+1}, \ldots, X_{2^{n+1}}, Y_{2^{n}}, \ldots, Y_{2^{n+1}-2}\right)\right\} Y_{2^{n+1}-1} ;
\end{gathered}
$$

Assim, dizemos que $R$ é Lie solúvel forte de classe $s$ se, e somente se, $\widetilde{\delta}_{s}(R)=0$, mas $\widetilde{\delta}_{s-1}(R) \neq 0$, ou equivalentemente, $R$ satisfaz a identidade de Lie solubilidade forte

$$
\bar{\delta}_{s}\left(X_{1}, \ldots, X_{2^{s}}, Y_{1}, \ldots, Y_{2^{s}-1}\right) \equiv 0
$$

onde $s$ é minimal. Uma outra condição equivalente é que existe a cadeia dos ideais de 
Poisson

$$
R=R_{0} \supset R_{1} \supset \cdots \supset R_{s}=0
$$

tais que os fatores $R_{i} / R_{i+1}, i=0, \ldots, s-1$, são abelianos e $s$ é minimal.

Observe também que

$$
\delta_{s}(R) \subseteq \widetilde{\delta}_{s}(R), \quad s \geq 0
$$

Em particular, a solubilidade forte de classe $d$ implica a solubilidade de classe $d$. A solubilidade de classe 1 é equivalente a solubilidade forte de classe 1 e é equivalente ao fato de $R$ ser uma álgebra de Lie abeliana.

\subsection{Lie nilpotência de álgebras de Poisson simétricas truncadas}

O nosso primeiro resultado principal é o seguinte.

Teorema 3.3.1 Seja L uma álgebra de Lie sobre um corpo de característica $p>0$. Considere sua álgebra de Poisson simétrica truncada $\mathbf{s}(L)$. As seguintes condições são equivalentes:

1. $\mathbf{s}(L)$ é Lie nilpotente forte;

2. $\mathbf{s}(L)$ é Lie nilpotente;

3. L é nilpotente e $\operatorname{dim} L^{2}<\infty$.

Seja $L$ uma álgebra de Lie sobre um corpo de característica positiva $p>3$. Usando a noção das potências de Lie superiores (veja acima), definimos subálgebras de dimensão de Poisson truncadas de $L$ como:

$$
D_{(n)}^{s}(L)=L \cap(\mathbf{s}(L))^{(n)}, \quad n \geq 0 .
$$

Pela afirmação 3 do Lema 4.3.1, obtemos a seguinte descrição dessas subálgebras, a qual é semelhante as fórmulas conhecidas para anéis de grupos e envelopantes restri$\operatorname{tas}(2.1)$ e $(2.2)$ :

$$
D_{(n)}^{s}(L)=\gamma_{n+1}(L), \quad n \geq 0 .
$$


Agora computamos as classes de nilpotência e nilpotência forte. Estes resultados são os análogos das fórmulas conhecidas para anéis de grupos e envelopantes restritas (veja Teorema 2.1.3 e Teorema 2.3.3).

Teorema 3.3.2 Seja L uma álgebra de Lie sobre um corpo de característica positiva $p>3$, tal que $\mathbf{s}(L)$ é Lie nilpotente. Os números seguintes são iguais:

1. a classe de Lie nilpotência forte de $\mathbf{s}(L)$;

2. a classe de Lie nilpotência de $\mathbf{s}(L)$;

3.

$$
1+(p-1) \sum_{n \geq 1} n \cdot \operatorname{dim}\left(\gamma_{n+1}(L) / \gamma_{n+2}(L)\right)
$$

Nos casos $p=2,3$ os números 1), 3) são iguais também.

Para características especiais $p=2,3$, o número acima produz uma cota superior para a classe de Lie nilpotência comum. Nestes casos, temos uma cota inferior para a classe de Lie nilpotência (veja Teorema 4.6.2):

$$
2+(p-1) \sum_{n \geq 2}(n-1) \cdot \operatorname{dim}\left(\gamma_{n+1}(L) / \gamma_{n+2}(L)\right) .
$$

\subsection{Solubilidade de álgebras de Poisson simétricas truncadas}

O nosso segundo resultado principal é o seguinte.

Teorema 3.4.1 Seja L uma álgebra de Lie sobre um corpo de característica $p \geq 3$. Considere sua álgebra de Poisson simétrica truncada $\mathbf{s}(L)$. As seguintes condições são equivalentes:

1. $\mathbf{s}(L)$ é Lie solúvel forte;

2. $\mathbf{s}(L)$ é Lie solúvel;

3. L é solúvel e $\operatorname{dim} L^{2}<\infty$.

No caso $p=2$ as condições 1 e 3 são equivalentes também. 


\subsubsection{A solubilidade no caso $p=2$}

Observe que, a resposta para a questão de solubilidade para anéis de grupo em característica 2 é vista muito bem (Teorema 2.1.2). Mas uma questão semelhante para álgebras envelopantes restritas é mais complicada (Teorema 2.3.4).

A resposta geral para a questão de solubilidade de $\mathbf{s}(L)$ no caso $p=2$ é uma questão em aberto. Como uma primeira abordagem, mostraremos que o problema de solubilidade da álgebra simétrica truncada no caso $p=2$ é diferente do caso de outras características.

Isto é, no caso quando char $K=2$, obtemos dois exemplos das álgebras simétricas truncadas que são solúveis mas não são solúveis forte, veja dois exemplos dados em Lema 5.2.1 e Lema 5.2.2.

Um fato próximo é que as álgebras Hamiltonianas $\mathbf{H}_{2}(K)$ e $\mathbf{h}_{2}(K)$ são solúveis mas não solúveis forte no caso char $K=2$ (Lema 5.2.3). Isto é um análogo de um fato bem conhecido que o anel $\mathrm{M}_{2}(K)$ das matrizes $2 \times 2$ sobre um corpo $K$, char $K=2$, é solúvel mas não é solúvel forte.

Finalmente, demonstramos a seguinte generalização do resultado de Shestakov (veja Teorema 2.5.2).

Teorema 3.4.2 Seja L uma álgebra de Lie sobre um corpo arbitrário $K$, e $S(L)$ sua álgebra simétrica. Então as seguintes condições são equivalentes:

1. L é abeliana;

2. $S(L)$ é Lie nilpotente forte;

3. $S(L)$ é Lie nilpotente;

4. $S(L)$ é Lie solúvel forte;

5. $S(L)$ é Lie solúvel (char $K \neq 2$ ). 


\section{Capítulo 4}

\section{Lie nilpotência. Demonstrações}

Neste capítulo demonstramos o nosso primeiro resultado principal sobre nilpotência da álgebra de Poisson simétrica truncada, isto é, nós demonstramos o seguinte teorema.

Teorema 3.3.1. Seja L uma álgebra de Lie sobre um corpo de característica $p>0$. Considere sua álgebra de Poisson simétrica truncada $\mathbf{s}(L)$. As seguintes condições são equivalentes:

1. $\mathbf{s}(L)$ é Lie nilpotente forte;

2. $\mathbf{s}(L)$ é Lie nilpotente;

3. L é nilpotente e $\operatorname{dim} L^{2}<\infty$.

\subsection{Delta-conjuntos e identidades de Poisson}

No caso de grupos, delta-conjuntos foram introduzidos por Passman [34]. Seja Gum grupo, então denote

$$
g^{G}=\left\{h^{-1} g h \mid h \in G\right\}, \quad g \in G,
$$


a classe de conjugação de $g$ em $G$,

$$
\Delta_{n}(G)=\left\{g \in G|| g^{G} \mid=n\right\}, \quad n \geq 1
$$

e

$$
\Delta(G)=\bigcup_{n=1}^{\infty} \Delta_{n}(G) .
$$

O conjunto $\Delta(G)$ é chamado o conjunto dos $\mathcal{F C}$-elementos. Esta noção é um instrumento poderoso para estudar identidades nos anéis de grupos $K[G]$, veja [4].

No caso de álgebras de Lie, eles foram introduzidos por Bahturin [1]. No que segue, $L$ é uma álgebra de Lie (restrita) sobre um corpo $K$ de característica $p>0$. Também recordaremos a noção de delta-conjuntos para álgebras de Lie. Se $L$ é uma álgebra de Lie, definimos o conjunto dos elementos de "largura finita" com respeito aos colchetes de Lie como

$$
\begin{aligned}
{[L, x] } & =\langle[y, x] \mid y \in L\rangle_{K}, \quad x \in L ; \\
\Delta_{n}(L) & =\left\{x \in L \mid \operatorname{dim}_{K}[L, x] \leq n\right\}, \quad n \geq 0 \\
\Delta(L) & =\bigcup_{n=0}^{\infty} \Delta_{n}(L)=\left\{x \in L \mid \operatorname{dim}_{K}[L, x]<\infty\right\} .
\end{aligned}
$$

Os delta-conjuntos da álgebra de Lie têm um papel chave no estudo das identidades de álgebras envelopantes como é conhecido em [4]. Note que os conjuntos $\Delta_{n}(L)$ não são subálgebras, pois eles não são nem mesmo subespaços em geral. As propriedades básicas dos delta-conjuntos são dadas no seguinte resultado.

Lema 4.1.1 ([4]) Seja L uma álgebra de Lie. Então

1. cada $\Delta_{n}(L), n \in \mathbb{N}$, é invariante com respeito a multiplicação por escalar;

2. se $x \in \Delta_{i}(L), y \in \Delta_{j}(L)$ então $\alpha x+\beta y \in \Delta_{i+j}(L)$, onde $\alpha, \beta \in K$;

3. se $x \in \Delta_{i}(L), y \in L$ então $[x, y] \in \Delta_{2 i}(L)$;

4. seja $x \in \Delta_{i}(L)$ e suponha que $L$ é uma álgebra de Lie restrita; então $x^{[p]} \in \Delta_{i}(L)$; 
5. $\Delta(L)$ é um ideal (restrito) de L.

No que segue, iremos fazer uso do próximo resultado sobre aplicações bilineares.

Teorema 4.1.2 (P.M. Neumann, 1970, [30], [1]) Sejam U, V,W espaços vetoriais sobre um corpo $K$ e seja $\varphi: U \times V \rightarrow W$ uma aplicação bilinear. Suponha que para todos $u \in U$ e $v \in V, \operatorname{dim} \varphi(u, V) \leq m e \operatorname{dim} \varphi(U, v) \leq l$. Então

$$
\operatorname{dim}\langle\varphi(U, V)\rangle_{K} \leq m l
$$

Concluímos esta seção com um resultado o qual parte de uma simples propriedade dos delta-conjuntos.

Lema 4.1.3 ([42]) Seja L uma álgebra de Lie. Então

1. Se I é um ideal de dimensão finita de $L$, então $\Delta(L / I)=(\Delta(L)+I) / I$;

2. se $H$ é uma subálgebra de codimensão finita em $L$, então $\Delta(H)=\Delta(L) \cap H$.

Demonstração. A afirmação 1 segue do fato que $(\Delta(L)+I) / I=\Delta(L) / \Delta(L) \cap I$ e pela aplicação $\varphi: \Delta(L) / \Delta(L) \cap I \rightarrow \Delta(L / I)$ definida por $\varphi(x+\Delta \cap I)=x+I$ ser um isomorfismo. Seja $x \in L$, denote $\bar{L}=L / I$ e $\bar{x}$ a imagem de $x$ em $I$, então $\operatorname{dim}[\bar{x}, \bar{L}] \leq$ $\operatorname{dim}[x, L] \leq \operatorname{dim}[\bar{x}, \bar{L}]+\operatorname{dim} I$. Como por hipótese $\operatorname{dim} I<\infty$, a imagem de $x$ tem largura finita em $\bar{L}$ se, e somente se, $x$ em $L$.

Agora, a afirmação 2, como $\operatorname{dim} L / H=n<\infty$, existem $x_{1}, x_{2}, \ldots, x_{n} \in L \backslash H$ tais que $L=\left\langle x_{1}, x_{2}, \ldots, x_{n}\right\rangle_{K} \oplus H$. Seja $h \in H$, então

$$
[L, h]=\left\langle\left[x_{1}, h\right], \ldots,\left[x_{n}, h\right]\right\rangle_{K} \oplus[H, h]
$$

e $\operatorname{dim}[L, h]<\infty$ se, e somente se, $\operatorname{dim}[H, h]<\infty$, isto é $h \in \Delta(L)$ se, e somente se, $h \in \Delta(H)$. Portanto, $h \in \Delta(L) \cap H$ se, e somente se, $h \in \Delta(H)$.

Mais delta-conjuntos foram introduzidos no estudo das identidades em álgebras envelopantes restritas $u(L)$ de uma superálgebra de Lie restrita [38] e no estudo das identidades nos produtos smash $u(L) \# K[G]$ e $U(L) \# K[G]$, veja [5]. 
Quando $L$ é uma álgebra de Poisson, consideremos os delta-conjuntos com respeito aos colchetes de Poisson $\{$,$\} .$

O seguinte resultado tem um papel crucial em nosso trabalho.

Teorema 4.1.4 (Giambruno, Petrogradsky, 2006, [15]) Seja L uma álgebra de Lie (restrita) sobre um corpo $K$ qualquer. Suponha que a álgebra simétrica $S(L)$ (álgebra simétrica truncada $\mathbf{s}(L)$ ) satisfaz uma identidade de Poisson multilinear. Então existem inteiros $n, N$ tais que

$$
\operatorname{dim}_{K} L / \Delta_{N}(L)<n
$$

As identidades de Lie nilpotência (forte) e solubilidade (forte) são multilineares e nós aplicamos este resultado para reduzir nossos casos para investigação mais detalhada.

\subsection{Filtrações em álgebras de Lie e álgebras simétricas truncadas}

Seja $L$ uma álgebra de Lie (restrita) sobre um corpo $K$ de característica positiva $p$. Uma filtração de $\mathbf{s}(L)$ é uma sequência decrescente de subespaços de $\mathbf{s}(L)$

$$
\mathbf{s}(L)=E_{0} \supseteq E_{1} \supseteq \cdots \supseteq E_{n} \supseteq \cdots, \quad \bigcap_{n \geq 0} E_{n}=\{0\}
$$

satisfazendo $E_{i} \cdot E_{j} \subseteq E_{i+j}$ e $\left\{E_{i}, E_{j}\right\} \subseteq E_{i+j}$, para todos $i, j \geq 0$. Tal sequência nos dá de maneira natural uma sequência decrescente de subespaços

$$
L=L_{0} \supseteq L_{1} \supseteq \cdots \supseteq L_{n} \supseteq \cdots, \bigcap_{n \geq 0} L_{n}=\{0\}
$$

definindo por $L_{n}=L \cap E_{n}$, para todo $n \geq 0$. Segue pela definição do produto $\{\cdot, \cdot\}$ em $\mathbf{s}(L)$ que

$$
\left\{L_{i}, L_{j}\right\} \subseteq L_{i+j}, \quad i, j \geq 0
$$

Dizemos que uma sequência de subespaços que satisfaz estas propriedades é uma filtração de $L$. Observe que cada $L_{i}$ não necessariamente é uma subálgebra (restrita) de $L$. 
Vimos que uma filtração de $\mathbf{s}(L)$ dá uma filtração para $L$. De fato, o inverso também é verdade. Suponha que $\left\{L_{n} \mid n \geq 0\right\}$ é qualquer filtração de $L$. Para cada $x \in L$ definimos a cota de $x$, a qual iremos denotar por $\nu(x)$, como sendo o maior índice $n$ tal que $x \in L_{n}$. Agora, para cada inteiro $m \geq 0$, seja $E_{m} \subseteq \mathbf{s}(L)$ o $K$-espaço linear gerado pelos produtos da forma

$$
x_{1} x_{2} \cdots x_{l}
$$

onde $x_{i} \in L$, com $\nu\left(x_{1}\right)+\nu\left(x_{2}\right)+\cdots+\nu\left(x_{l}\right) \geq m$.

Veremos que $\left\{E_{m} \mid m \geq 0\right\}$ é uma filtração de $\mathbf{s}(L)$.

Lema 4.2.1 Seja $E_{m}, m \geq 0$ definido como acima, então para todos $n, m \geq 0$ temos:

1. $E_{n} \cdot E_{m} \subseteq E_{n+m}$;

2. $\left\{E_{n}, E_{m}\right\} \subseteq E_{n+m}$.

Demonstração. Seja $E_{n}$ o $K$-espaço linear gerado por todos os produtos da forma $x_{1} \cdots x_{l}$ onde $x_{1}, \ldots, x_{l} \in L, \sum_{i=1}^{l} \nu\left(x_{i}\right) \geq n$ e $E_{m}$ seja $K$-espaço linear gerado por todos os produtos da forma $y_{1} \cdots y_{k}$ onde $y_{1}, \ldots, y_{k} \in L, \sum_{j=1}^{k} \nu\left(y_{j}\right) \geq m$. A parte 1 segue pela comutatividade do produto $\cdot$ em $\mathbf{s}(L)$.

Agora, sejam $a=x_{1} \cdots x_{l} \in E_{n}$ e $b=y_{1} \cdots y_{k} \in E_{m}$, temos pela regra de Leibnitz

$$
\{a, b\}=\sum_{i, j} x_{1} \cdots \widehat{x_{i}} \cdots x_{l} \cdot y_{1} \cdots \widehat{y_{j}} \cdots y_{k}\left\{x_{i}, y_{j}\right\}
$$

como $\nu\left(\left\{x_{i}, y_{j}\right\}\right) \geq \nu\left(x_{i}\right)+\nu\left(y_{j}\right)$, segue que $\{a, b\} \in E_{m+n}$ e obtemos a parte 2 .

A relação entre as duas filtrações $\left\{L_{n} \mid n \geq 0\right\}$ e $\left\{E_{n} \mid n \geq 0\right\}$ é demonstrada pelo seguinte lema.

Lema 4.2.2 Seja $\left\{L_{n} \mid n \geq 0\right\}$ uma filtração de uma álgebra de Lie L sobre um corpo K de característica $p>0$ e seja $\left\{x_{i} \mid i \in I\right\}$ uma base ordenada de L escolhida de maneira que $L_{n}=\left\langle x_{i} \mid \nu\left(x_{i}\right) \geq n\right\rangle_{K}, n \geq 0$. Seja $\left\{E_{n} \mid n \geq 0\right\}$ a filtração de $\mathbf{s}(L)$ induzida por $\left\{L_{n} \mid n \geq 0\right\}$. Então as seguintes afirmações são válidas para cada $n \geq 0$. 
1. $E_{n}=\langle\eta \mid \nu(\eta) \geq n\rangle_{K}$, onde $\eta=x_{1}^{a_{1}} x_{2}^{a_{2}} \cdots x_{l}^{a_{l}}, \nu(\eta)=\sum_{j=1}^{l} a_{j} \nu\left(x_{j}\right), l \geq 0$ e, $0 \leq$ $a_{j} \leq p-1$, para cada $j$;

2. $\{\eta \mid \nu(\eta)=n\}$ é uma $K$-base de $E_{n}$ módulo $E_{n+1}$;

3. o conjunto de todos tais monômios $\eta$ é uma base de $\mathbf{s}(L)$;

4. $L_{n}=L \cap E_{n}$. (Assim, retornamos à filtração original).

Demonstração. Seja $n \geq 0$ fixado e seja $E_{n, k}$ o $K$-espaço linear gerado pelos produtos da forma $y_{1} y_{2} \cdots y_{l}$, onde $y_{1}, y_{2}, \ldots, y_{l} \in L, \sum_{j=1}^{l} \nu\left(y_{j}\right) \geq n$ e $l \leq k$. Afirmamos que $E_{n, k}$ é gerado pelo conjunto dos monômios

$$
x_{1}^{a_{1}} x_{2}^{a_{2}} \cdots x_{l}^{a_{l}}, \quad \sum_{j=1}^{l} a_{j} \nu\left(x_{j}\right) \geq n, \quad 0 \leq a_{j} \leq p-1, j=1, \ldots, l, \quad \sum_{j=1}^{l} a_{j} \leq k .
$$

Por definição, estes elementos estão contidos em $E_{n, k}$. Provaremos que eles geram $E_{n, k}$ por indução sobre $k \geq 1$. O caso $k=1$ segue por nossa escolha da base

$$
E_{n, 1}=\langle\eta=y \in L \mid \nu(y) \geq n\rangle_{K}=L_{n} .
$$

Agora, assuma que a afirmação vale para todo $j<k$. Sejam $y_{1}, y_{2}, \ldots, y_{k} \in L$ satisfazendo $\sum_{j=1}^{k} \nu\left(y_{j}\right) \geq n$. Como $y_{1} y_{2} \cdots y_{k-1} \in E_{\sum_{j=1}^{k-1} \nu\left(y_{j}\right), k-1}$, por hipótese de indução, obtemos que $y_{1} y_{2} \cdots y_{k-1}$ é uma combinação linear de monômios $x_{1}^{a_{1}} x_{2}^{a_{2}} \cdots x_{l}^{a_{l}}, 0 \leq a_{j} \leq p-1$ para cada $j, \sum_{j=1}^{l} a_{j} \nu\left(x_{j}\right) \geq \sum_{j=1}^{k-1} \nu\left(y_{j}\right)$ e $\sum_{j=1}^{l} a_{j} \leq k-1$. Também $y_{k}$ é expresso via $x_{j} \operatorname{com} \nu\left(x_{j}\right) \geq \nu\left(y_{k}\right)$. O produto é comutativo, logo

$$
x_{1}^{a_{1}} x_{2}^{a_{2}} \cdots x_{l}^{a_{l}} x_{j}=x_{1}^{a_{1}} \cdots x_{j}^{a_{j}+1} \cdots x_{l}^{a_{l}} \in E_{n, k} .
$$

Obtemos que $y_{1} y_{2} \cdots y_{k}$ é gerado pelos monômios desejados $x_{1}^{a_{1}} \cdots x_{j}^{a_{j}+1} \cdots x_{l}^{a_{l}}$. A afirmação segue, a menos de $a_{j}=p-1$. Tais termos desaparecem porque $x_{j}^{a_{j}+1}=x_{j}^{p}=0$.

Como $E_{n}=\sum_{k \geq 1} E_{n, k}$, a parte 1 segue.

A parte 2 segue de 1 e por $E_{n} \supseteq E_{n+1}$, portanto $E_{n} / E_{n+1}=\langle\eta \mid \nu(\eta)=n\rangle_{K}$.

A parte 3 é clara. 
Agora, observe que

$$
E_{n} \cap L=\left\langle\eta=x_{1}^{a_{1}} x_{2}^{a_{2}} \cdots x_{l}^{a_{l}} \mid \sum_{j=1}^{l} a_{j}=1, \nu(\eta) \geq n\right\rangle=L_{n}
$$

e obtemos a parte 4 .

Lema 4.2.3 Seja $R$ uma álgebra de Poisson arbitrária. Então as potências de Lie superiores $\left\{R^{(n)} \mid n \geq 0\right\}$ formam uma filtração, i.e. $R^{(i)} \cdot R^{(j)} \subset R^{(i+j)} e\left\{R^{(i)}, R^{(j)}\right\} \subset R^{(i+j)}$ para todo $i, j \geq 0$.

Demonstração. Primeiro, consideramos o produto associativo comutativo. Procedemos por indução sobre $j$. Seja $j=0$, então $R^{(0)}=R$ e não há nada a provar. Suponha que a afirmação é válida para $j-1 \geq 0$. Usaremos a regra de Leibnitz na forma $a\{b, c\}=$ $\{a b, c\}-b\{a, c\}$ e aplicaremos o argumento de indução:

$$
\begin{aligned}
R^{(i)} \cdot R^{(j)} & =R^{(i)}\left\{R^{(j-1)}, R\right\} R \\
& \subseteq\left\{R^{(i)} R^{(j-1)}, R\right\} R+R^{(j-1)}\left\{R^{(i)}, R\right\} R \\
& \subseteq\left\{R^{(i+j-1)}, R\right\} R+R^{(j-1)} R^{(i+1)} \\
& \subseteq R^{(i+j)} .
\end{aligned}
$$

A afirmação para o produto de Lie também segue por indução. Seja $j=0$, então $\left\{R^{(i)}, R^{(0)}\right\} \subseteq\left\{R^{(i)}, R\right\} \subseteq R^{(i+1)} \subseteq R^{(i)}$. Suponha que a afirmação é válida para $j-1 \geq 0$. Usando a regra de Leibnitz, e a identidade de Jacobi na forma $\{x,\{y, z\}\}=\{\{z, x\}, y\}+$ $\{\{x, y\}, z\}$ e a inclusão anterior, temos

$$
\begin{aligned}
\left\{R^{(i)}, R^{(j)}\right\} & =\left\{R^{(i)},\left\{R^{(j-1)}, R\right\} \cdot R\right\} \\
& \subseteq\left\{R^{(i)}, R\right\} \cdot\left\{R^{(j-1)}, R\right\}+\left\{R^{(i)},\left\{R^{(j-1)}, R\right\}\right\} \cdot R \\
& \subseteq R^{(i+1)} \cdot R^{(j)}+\left(\left\{\left\{R, R^{(i)}\right\}, R^{(j-1)}\right\}+\left\{\left\{R^{(i)}, R^{(j-1)}\right\}, R\right\}\right) \cdot R \\
& \subseteq R^{(i+j+1)}+\left(\left\{R^{(i+1)}, R^{(j-1)}\right\}+\left\{R^{(i+j-1)}, R\right\}\right) \cdot R \\
& \subseteq R^{(i+j+1)}+R^{(i+j)} \cdot R+R^{(i+j)} R \\
& \subseteq R^{(i+j)} .
\end{aligned}
$$




\subsection{Lie nilpotência forte da álgebra simétrica trun- cada}

Agora, assumiremos que $R=\mathbf{s}(L)$. Mudando a enumeração dos termos da série central inferior obtemos a seguinte filtração:

$$
L=L_{0} \supseteq L_{1} \supseteq \cdots \supseteq L_{n} \supseteq \cdots, \quad \text { onde } \quad L_{n}=\gamma_{n+1}(L), n \geq 0 .
$$

Pelo próximo resultado, esta filtração alterada $\left\{L_{n} \mid n \geq 0\right\}$ induz a filtração de $\mathbf{s}(L)$ pelas potências superiores de Lie $\left\{\mathbf{s}(L)^{(n)} \mid n \geq 0\right\}$, veja a seção anterior.

Lema 4.3.1 Seja L uma álgebra de Lie arbitrária sobre um corpo de característica p, e $\gamma_{n}(L), n \geq 1$, os termos da série central inferior de L. Então

1. as potências de Lie superiores para a álgebra simétrica truncada $\mathbf{s}(L)$ são dadas por

$$
\mathbf{s}(L)^{(n)}=\sum_{\substack{\left(m_{1}-1\right)+\cdots+\left(m_{s}-1\right) \geq n \\ 1 \leq m_{1} \leq m_{2} \leq \cdots \leq m_{s}}} \gamma_{m_{1}}(L) \gamma_{m_{2}}(L) \cdots \gamma_{m_{s}}(L), \quad n \geq 0 ;
$$

2. $\left\{\mathbf{s}(L)^{(n)} \mid n \geq 0\right\}$ é a filtração $\left\{E_{n} \mid n \geq 0\right\}$, a qual é a filtração induzida pela filtração alterada $\left\{L_{i}=\gamma_{i+1}(L) \mid i \geq 0\right\}$;

3. $\mathbf{s}(L)^{(n)} \cap L=\gamma_{n+1}(L), n \geq 0$.

Demonstração. Para a parte 1, considere uma quantidade finita de inteiros $\left\{m_{j}\right\}$ tais que $m_{j} \geq 1$ e $\sum\left(m_{j}-1\right) \geq n$. Para provar que o lado direito está contido no lado esquerdo, aplicamos a expressão (3.2) do capítulo anterior, de modo que $\gamma_{m}(L) \subseteq$ $\gamma_{m}(\mathbf{s}(L)) \subseteq \mathbf{s}(L)^{(m-1)}$ para $m \geq 1$. Aplicamos parte 1 do Lema 4.2 .3 e chegamos em

$$
\begin{aligned}
\gamma_{m_{1}}(L) \gamma_{m_{2}}(L) \cdots \gamma_{m_{s}}(L) & \subseteq \mathrm{s}(L)^{\left(m_{1}-1\right)} \mathbf{s}(L)^{\left(m_{2}-1\right)} \cdots \mathbf{s}(L)^{\left(m_{s}-1\right)} \\
& \subseteq \mathbf{s}(L)^{\left(\left(m_{1}-1\right)+\left(m_{2}-1\right)+\cdots+\left(m_{s}-1\right)\right)} \\
& \subseteq \mathbf{s}(L)^{(n)}
\end{aligned}
$$

Portanto, obtemos a inclusão 


$$
\sum_{\substack{\left(m_{1}-1\right)+\cdots+\left(m_{s}-1\right) \geq n \\ 1 \leq m_{1} \leq m_{2} \leq \cdots \leq m_{s}}} \gamma_{m_{1}}(L) \gamma_{m_{2}}(L) \cdots \gamma_{m_{s}}(L) \subseteq \mathbf{s}(L)^{(n)}, n \geq 0 .
$$

Para provar a inclusão contrária, usaremos indução sobre $n$. O caso $n=0$ é evidente. Fazemos uma observação, seja $a \in \gamma_{m_{i}}(L)$ um comutador de comprimento no mínimo $m_{i}$, e pegamos um monômio $w=b_{1} \cdots b_{t} \in \mathbf{s}(L), b_{j} \in L$, temos

$$
\{a, w\}=\sum_{j=1}^{t} b_{1} \cdots \widehat{b_{j}} \cdots b_{t}\left\{a, b_{j}\right\}
$$

onde $\left\{a, b_{j}\right\} \in \gamma_{m_{i}+1}(L), b_{j} \in L=\gamma_{1}(L)$. Observe que o último fator não influencia na igualdade com respeito a soma. Assim, ganhamos a inclusão contrária

$$
\begin{aligned}
\mathbf{s}(L)^{(n)} & =\left\{\mathbf{s}(L)^{(n-1)}, \mathbf{s}(L)\right\} \mathbf{s}(L) \\
& \subseteq\left\{\sum_{\sum\left(m_{i}-1\right) \geq n-1} \gamma_{m_{1}}(L) \cdots \gamma_{m_{s}}(L), \mathbf{s}(L)\right\} \mathbf{s}(L) \\
& \subseteq \sum_{\sum\left(n_{i}-1\right) \geq n} \gamma_{n_{1}}(L) \cdots \gamma_{n_{r}}(L), \quad n \geq 1 .
\end{aligned}
$$

Podemos ordenar os índices pois $\mathbf{s}(L)$ é comutativa.

A afirmação 2 segue pela definição da filtração alterada e pela afirmação 1 .

Finalmente, a afirmação 3 segue pela afirmação 4 do Lema 4.2.2.

Seja $\left\{e_{i j} \mid i \geq 0, j \in J_{i}\right\}$ uma base ordenada de $L$ escolhida de modo que $\left\{e_{i j} \mid j \in J_{i}\right\}$ é uma base para $L_{i}=\gamma_{i+1}(L)$ módulo $L_{i+1}=\gamma_{i+2}(L)$, para todo $i \geq 0$. Temos a seguinte função $\operatorname{cota} \nu\left(e_{i j}\right)=i, i \geq 0, j \in J_{i}$. Pelo Lema 4.2.2, temos a seguinte afirmação. 
Corolário 4.3.2 O espaço $\mathbf{s}(L)^{(n)}$ módulo $\mathbf{s}(L)^{(n+1)}$ tem a seguinte base para todo $n \geq 0$ :

$$
\begin{gathered}
\left\langle\eta=e_{01}^{\alpha_{01}} \ldots e_{0 m_{0}}^{\alpha_{0 m_{0}}} e_{11}^{\alpha_{11}} \ldots e_{1 m_{1}}^{\alpha_{1 m_{1}}} \ldots e_{s 1}^{\alpha_{s 1}} \ldots e_{s m_{s}}^{\alpha_{s m_{s}}}\right| \\
\left.\nu(\eta)=\sum_{\substack{1 \leq i \leq s \\
1 \leq j \leq m_{i}}} i \alpha_{i j}=n ; 0 \leq \alpha_{i j} \leq p-1, s \geq 0\right\rangle_{K} .
\end{gathered}
$$

Demonstração. A prova segue da afirmação 2 do Lema 4.2.2.

Demonstração da equivalência 1) $\Leftrightarrow 3$ ) do Teorema 3.3.1.

Mostraremos que 1 implica 3. Seja $\mathbf{s}(L)$ Lie nilpotente forte, então $\mathbf{s}(L)^{(m)}=0$, para algum inteiro positivo $m$. Usando a afirmação 3 do Lema 4.3.1, chegamos na inclusão $\gamma_{m+1}(L) \subset \mathbf{s}(L)^{(m)}=0$, assim $L$ é uma álgebra de Lie nilpotente. De maneira a chegar numa contradição, assuma que $\operatorname{dim} L^{2}=\infty$. Construímos uma base de $L$ como acima. Denote por $f_{i}$ os elementos da base pertencentes a $L^{2}$, existe uma quantidade infinita de tais elementos. Temos $\nu\left(f_{i}\right) \geq 1$. Então $0 \neq v=f_{1} \cdots f_{m} \in E_{m}=\mathbf{s}(L)^{(m)}$, para qualquer $m \geq 1$. Assim, $\mathbf{s}(L)$ não é Lie nilpotente forte. Uma contradição.

Iremos mostrar que 3) implica 1). Suponha que $L$ é nilpotente e $L^{2}$ é de dimensão finita. Neste caso, denotaremos por $d_{i}=\operatorname{dim} \gamma_{i+1}(L) / \gamma_{i+2}(L)$, que é um finito para todo $i \geq 1$, e vamos supor que $\left\{e_{i 1}, e_{i 2}, \ldots, e_{i d_{i}}\right\}$ é uma base para $\gamma_{i+1}(L)$ módulo $\gamma_{i+2}(L)$, para todo $i \geq 1$. Considere

$$
\eta=e_{01}^{\alpha_{01}} \ldots e_{0 m_{0}}^{\alpha_{0 m_{0}}} e_{11}^{\alpha_{11}} \ldots e_{1 m_{1}}^{\alpha_{1 m_{1}}} \ldots e_{s 1}^{\alpha_{s 1}} \ldots e_{s m_{s}}^{\alpha_{s m_{s}}}
$$

um elemento da base, com $\nu(\eta)=\sum_{\substack{0 \leq i \leq s \\ 1 \leq j \leq m_{i}}} i \alpha_{i j}$. Note que

$$
\nu(\eta) \leq(p-1) \sum_{n \geq 1} n d_{n}
$$

onde a soma é finita. Pondo $N=(p-1) \sum_{n \geq 1} n d_{n}$ portanto, segue que $\mathbf{s}(L)^{(N+1)}=$ $E_{N+1} \equiv\{0\}$, mas $E_{N} \not \equiv\{0\}$ porque temos $0 \neq v=e_{11}^{p-1} \cdots e_{s m_{s}}^{p-1} \in E_{N} \operatorname{com} \nu(v)=$ $(p-1) \sum_{n \geq 1} n d_{n}=N$. Então, $\mathbf{s}(L)$ é Lie nilpotente forte de classe $N+1$.

Nós de fato computamos a classe de Lie nilpotência forte de $\mathbf{s}(L)$, isto é obtemos a equivalência 1) $\Leftrightarrow 3$ ) do Teorema 3.3 .2 como a seguir. 
Corolário 4.3.3 Seja L uma álgebra de Lie sobre um corpo de característica positiva p. Assuma que $L$ é nilpotente e $\operatorname{dim} L^{2}<\infty$. Então $\mathbf{s}(L)$ é Lie nilpotente forte de classe

$$
1+(p-1) \sum_{n \geq 1} n \cdot \operatorname{dim}\left(\gamma_{n+1}(L) / \gamma_{n+2}(L)\right)
$$

\subsection{Lie nilpotência da álgebra simétrica truncada}

A conclusão da demonstração da Teorema 3.3.1. Segue da inclusão (3.2), que a parte 1) implica em 2).

Resta-nos demonstrar que 2) implica em 3). Como $L$ é claramente nilpotente, restanos mostrar que $L^{2}$ é de dimensão finita. Como $\mathbf{s}(L)$ satisfaz uma identidade de Poisson multilinear não trivial, pelo Teorema 4.1.4, existem inteiros $n, N$ tais que $\operatorname{dim}_{K} L / \Delta_{N}(L)<$ $n$, e pelas propriedades do Lema 4.1.1, chegamos que $\Delta(L)=\Delta_{M}(L)$ para algum inteiro $M$. Denotaremos $\Delta(L)$ simplesmente como $\Delta$. Assim, $L / \Delta$ é de dimensão finita, e usando o Teorema 4.1.2 para a aplicação bilinear $\Delta \times \Delta \rightarrow \Delta$, definida por $(u, v) \mapsto[u, v]$, para todo $u, v \in \Delta$, obtemos $\operatorname{dim} \Delta^{2} \leq M^{2}$. Assim, para provar que $L^{2}$ é de dimensão finita, é suficiente provar que $L=\Delta$. Iremos assumir o oposto, assumiremos que $L \neq \Delta$.

Considere a álgebra quociente de Lie $\bar{L}=L / \Delta^{2}$. Como $\operatorname{dim} \Delta^{2}<\infty$, pelo Lema 4.1.3, $\Delta(\bar{L})=\Delta / \Delta^{2}$. Portanto, $\Delta(\bar{L})$ é abeliano e $\bar{L} \neq \Delta(\bar{L})$. Assim, trocaremos $L$ por $\bar{L}$. Note que, como $\mathbf{s}(L)$ é Lie nilpotente, então $\mathbf{s}(H) \subseteq \mathbf{s}(L)$ é Lie nilpotente, onde $H$ é uma subálgebra de $L$. Então nós reduziremos nossas provas para $H=\langle x\rangle_{K} \oplus \Delta$, onde $x \in L \backslash \Delta$. Observe que $\Delta(H)=\Delta$, pelo Lema 4.1.3. Temos que $H$ é nilpotente, uma vez que $L$ é nilpotente, então existe um inteiro $n$ tal que $(\operatorname{ad} x)^{n}(H)=0$. A seguir, reduziremos nosso argumentos para o caso $(\operatorname{ad} x)^{2}(H)=0$.

De fato, para cada inteiro positivo $m$, denotaremos por $\Delta_{(m)}$ o $m$-ésimo ideal definido por $\Delta_{(0)}=\Delta$ e $\Delta_{(m+1)}=\left\{x, \Delta_{(m)}\right\}, m \geq 0$. Então obtemos uma cadeia destes ideais

$$
\Delta=\Delta_{(0)} \supseteq \Delta_{(1)}=\{x, \Delta\}=H^{\prime} \supseteq \Delta_{(2)} \supseteq \cdots \supseteq \Delta_{(n)}=0 .
$$

Como $\operatorname{dim} \Delta_{(1)}=\infty$, existe algum $i$ (onde $1 \leq i<n$ ) tal que $\operatorname{dim} \Delta_{(i)} / \Delta_{(i+1)}=\infty$. Seja $\bar{H}$ a álgebra de Lie $\left(\langle x\rangle_{K} \oplus \Delta_{(i-1)}\right) / \Delta_{(i+1)}$, e seja $\bar{x}$ a imagem de $x$ em $\bar{H}$. Como 
$\Delta_{(i)} / \Delta_{(i+1)}$ tem dimensão infinita, temos

$$
\begin{aligned}
\operatorname{dim}\{\bar{x}, \bar{H}\} & =\operatorname{dim}\left(\left\{x, \Delta_{(i-1)}\right\}+\Delta_{(i+1)}\right) / \Delta_{(i+1)} \\
& =\operatorname{dim}\left(\Delta_{(i)}+\Delta_{(i+1)}\right) / \Delta_{(i+1)}=\infty
\end{aligned}
$$

Por construção, $(\operatorname{ad} \bar{x})^{2}(\bar{H})=0$.

Agora, temos o caso $H=\langle x\rangle_{K} \oplus \Delta$, onde $\Delta$ é abeliano $(\operatorname{ad} x)^{2} \Delta=0, \operatorname{dim}\{x, \Delta\}=\infty$. Encontramos elementos $\left\{y_{1}, y_{2}, \ldots\right\}$ em $\Delta$ tais que $\left\{x, y_{1}\right\}=z_{1},\left\{x, y_{2}\right\}=z_{2}, \ldots$, são linearmente independentes. Afirmamos que

$$
\left\{x, x y_{1}, x y_{2}, \ldots, x y_{n}\right\}=x z_{1} z_{2} \cdots z_{n}
$$

para todo $n \geq 1$. A prova desta afirmação é feita por indução sobre $n$. O caso $n=1$ segue pela regra de Leibnitz. Vamos supor que a afirmação é válida para $n-1$. Aplicamos a regra de Leibnitz novamente e recordando que $z_{1}, z_{2}, \ldots$ são centrais, chegamos que em $\mathbf{s}(H)$

$$
\begin{aligned}
\left\{x, x y_{1}, x y_{2}, \ldots, x y_{n}\right\}= & \left\{\left\{x, x y_{1}, x y_{2}, \ldots, x y_{n-1}\right\}, x y_{n}\right\} \\
= & \left\{x z_{1} z_{2} \cdots z_{n-1}, x y_{n}\right\} \\
= & y_{n}\left\{x z_{1} z_{2} \cdots z_{n-1}, x\right\} \\
& +x\left\{x z_{1} z_{2} \cdots z_{n-1}, y_{n}\right\} \\
= & x z_{1} z_{2} \cdots z_{n-1}\left\{x, y_{n}\right\} \\
= & x z_{1} z_{2} \cdots z_{n-1} z_{n} .
\end{aligned}
$$

Mas o lado direito não é zero, pelo PBW-teorema para $\mathbf{s}(H)$. Mas isto contradiz nossa afirmação que $\mathbf{s}(H)$ é Lie nilpotente. A contradição prova que $L=\Delta$ e $L^{2}$ satisfaz a propriedade desejada.

Dessa forma, concluímos a demonstração de Teorema 3.3.1. 


\subsection{Produtos de comutadores em álgebras de Poisson}

Observamos que a nilpotência forte implica a nilpotência de mesma classe. Então, a classe de nilpotência forte é uma cota superior para a classe de nilpotência ordinária. Efetivamente, a seguir provaremos que estas classes coincidem em vários casos de álgebras de Poisson que estamos estudando.

Produtos dos termos das séries centrais inferiores para álgebras associativas aparecem em trabalhos de vários matemáticos, os resultados são provados sem o conhecimento dos trabalhos anteriores. Provavelmente, os primeiros trabalhos sobre produtos de comutadores em álgebras associativas foram feitas por Latyshev em 1965 [25] e Volichenko em 1978 [57]. Veja mais trabalhos e.g. [17], [12].

No caso de álgebras associativas, a inclusão da Afirmação 1 do Teorema 4.5.1, provavelmente, foi primeiro estabelecida por Sharma e Shrivastava em 1990, [43]; a afirmação também foi demonstrada por Bapat e Jordan em 2013, [6] e por Grishin e Pchelintsev em 2015, [18]. Uma inclusão mais forte

$$
\gamma_{n}(A) \cdot \gamma_{m}(A) \subseteq \gamma_{n+m-1}(A)
$$

onde um dos inteiros $n, m \geq 2$ é ímpar, $A$ sendo uma álgebra associativa, está recentemente provada em [6].

Como foi observado em [42], a prova das versões associativas da Afirmação 2 do Teorema 4.5.1 está implicitamente contida em [43], aqui os autores provam o resultado em caso de anéis de grupos.

Uma inclusão fraca para produtos (a versão associativa do Lema 4.5.7) foi determinada primeiro por Latyshev em 1965, [25] e depois por Gupta e Levin em 1983, [19].

A seguinte afirmação é uma versão Poisson dos respectivos resultados para álgebras associativas. A validade deles não é automaticamente clara. Nós seguimos a abordagem elegante de Krasilnikov [23].

Teorema 4.5.1 Seja $R$ uma álgebra de Poisson arbitrária sobre um corpo $K$, char $K \neq$ 2,3. Então 
1. supondo que um dos inteiros $n, m \geq 1$ seja ímpar, então

$$
\gamma_{n}(R) \cdot \gamma_{m}(R) \subseteq \gamma_{n+m-1}(R) \cdot R, \quad n, m \geq 1
$$

2. para todo $x_{1}, \ldots, x_{n} \in R$, e $n, m \geq 2$ temos

$$
\left\{x_{1}, \ldots, x_{n}\right\}^{m} \in \gamma_{(n-1) m+1}(R) \cdot R \text {. }
$$

Procedemos por passos.

Lema 4.5.2 Seja $R$ uma álgebra de Poisson arbitrária. Então

1. para quaisquer $a_{1}, a_{2}, a_{3}, a_{4}, a_{5} \in R$, onde exatamente um dos elementos $a_{1}, a_{2}, a_{5}$ pertencem a $\gamma_{m}(R), m \geq 1$ temos

$$
\left\{a_{1}, a_{2}, a_{3}\right\}\left\{a_{4}, a_{5}\right\}+\left\{a_{1}, a_{2}, a_{4}\right\}\left\{a_{3}, a_{5}\right\} \in \gamma_{m+3}(R) R
$$

i.e. permutamos os elementos $a_{3}$ e $a_{4}$;

2. para qualquer $a_{5} \in \gamma_{m}(R), m \geq 1$ e quaisquer $a_{1}, a_{2}, a_{3}, a_{4} \in R$ temos

$$
\left\{a_{1}, a_{2}, a_{3}\right\}\left\{a_{4}, a_{5}\right\}+\left\{a_{1}, a_{4}, a_{3}\right\}\left\{a_{2}, a_{5}\right\} \in \gamma_{m+3}(R) R
$$

i.e. permutamos os elementos $a_{2}$ e $a_{4}$.

Demonstração. Aplicando a regra de Leibnitz, temos:

$$
\begin{aligned}
\left\{a_{1}, a_{2}, a_{3} a_{4}, a_{5}\right\}= & \left\{a_{3}\left\{a_{1}, a_{2}, a_{4}\right\}+\left\{a_{1}, a_{2}, a_{3}\right\} a_{4}, a_{5}\right\} \\
= & a_{3}\left\{a_{1}, a_{2}, a_{4}, a_{5}\right\}+\left\{a_{3}, a_{5}\right\}\left\{a_{1}, a_{2}, a_{4}\right\} \\
& +\left\{a_{1}, a_{2}, a_{3}\right\}\left\{a_{4}, a_{5}\right\}+\left\{a_{1}, a_{2}, a_{3}, a_{5}\right\} a_{4} .
\end{aligned}
$$

Considere o produto original, o primeiro e o último termo obtido. Pelas propriedades dos comutadores de Lie, segue que $\left\{a_{1}, a_{2}, a_{3} a_{4}, a_{5}\right\} \in \gamma_{m+3}(R),\left\{a_{1}, a_{2}, a_{4}, a_{5}\right\} \in \gamma_{m+3}(R)$, $\left\{a_{1}, a_{2}, a_{3}, a_{5}\right\} \in \gamma_{m+3}(R)$. Os dois termos do meio restantes dão exatamente a primeira afirmação 


$$
\left\{a_{1}, a_{2}, a_{3}\right\}\left\{a_{4}, a_{5}\right\}+\left\{a_{1}, a_{2}, a_{4}\right\}\left\{a_{3}, a_{5}\right\} \in \gamma_{m+3}(R) R .
$$

Considere a segunda afirmação. Pela regra de Leibnitz:

$$
\begin{aligned}
& \left\{a_{5}, a_{2} a_{4}, a_{1}, a_{3}\right\}=\left\{a_{2}\left\{a_{5}, a_{4}\right\}+\left\{a_{5}, a_{2}\right\} a_{4}, a_{1}, a_{3}\right\} \\
& =\left\{a_{2}\left\{a_{5}, a_{4}, a_{1}\right\}\right. \\
& \left.+\left\{a_{2}, a_{1}\right\}\left\{a_{5}, a_{4}\right\}+\left\{a_{5}, a_{2}\right\}\left\{a_{4}, a_{1}\right\}+\left\{a_{5}, a_{2}, a_{1}\right\} a_{4}, a_{3}\right\} \\
& =a_{2}\left\{a_{5}, a_{4}, a_{1}, a_{3}\right\} \\
& +\left\{a_{2}, a_{3}\right\}\left\{a_{5}, a_{4}, a_{1}\right\}+\left\{a_{2}, a_{1}\right\}\left\{a_{5}, a_{4}, a_{3}\right\} \\
& +\left\{a_{2}, a_{1}, a_{3}\right\}\left\{a_{5}, a_{4}\right\}+\left\{a_{5}, a_{2}\right\}\left\{a_{4}, a_{1}, a_{3}\right\} \\
& +\left\{a_{5}, a_{2}, a_{3}\right\}\left\{a_{4}, a_{1}\right\}+\left\{a_{5}, a_{2}, a_{1}\right\}\left\{a_{4}, a_{3}\right\} \\
&
\end{aligned}
$$

Observe que o produto original, o primeiro e o último termo acima pertencem a $\gamma_{m+3}(R) R$. Aplicamos a primeira afirmação às somas na segunda e quarta linhas. Cada uma delas pertencem a $\gamma_{m+3}(R) R$, pois permutamos os dois elementos $a_{3}, a_{1}$ em ambos os casos.

Concluímos que a soma dos dois elementos na terceira linha da última igualdades, nos dá a inclusão desejada:

$$
\begin{aligned}
\left\{a_{2}, a_{1}, a_{3}\right\}\left\{a_{5}, a_{4}\right\}+\left\{a_{5}, a_{2}\right\} & \left\{a_{4}, a_{1}, a_{3}\right\} \\
& =\left\{a_{1}, a_{2}, a_{3}\right\}\left\{a_{4}, a_{5}\right\}+\left\{a_{1}, a_{4}, a_{3}\right\}\left\{a_{2}, a_{5}\right\} \in \gamma_{m+3}(R) R .
\end{aligned}
$$

Lema 4.5.3 Seja $R$ uma álgebra de Poisson arbitrária. Então para qualquer $a_{5} \in \gamma_{m}(R)$, com $m \geq 1$, e quaisquer $a_{1}, a_{2}, a_{3}, a_{4} \in R$ temos

$$
3\left\{a_{1}, a_{2}, a_{3}\right\}\left\{a_{4}, a_{5}\right\} \in \gamma_{m+3}(R) R .
$$

Demonstração. Considere o produto $W=\left\{a_{1}, a_{2}, a_{3}\right\}\left\{a_{4}, a_{5}\right\}$. Observe que, módulo $\gamma_{m+3}(R) R$, ele é antissimétrico nas letras $a_{3}, a_{4}$ (Lema 4.5.2, primeira afirmação) antis- 
simétrico nas letras $a_{2}, a_{4}$ (Lema 4.5.2, segunda afirmação). Portanto, ele é antissimétrico nas letras $a_{2}, a_{3}$ módulo $\gamma_{m+3}(R) R$. Antissimetria em $a_{1}, a_{2}$ segue pela anticomutatividade. Assim, $W$ é antissimétrico nas letras $a_{1}, a_{2}, a_{3}$. Considere só as permutações pares

$$
\begin{aligned}
\left\{a_{1}, a_{2}, a_{3}\right\}\left\{a_{4}, a_{5}\right\}+\left\{a_{2}, a_{3}, a_{1}\right\}\left\{a_{4}, a_{5}\right\}+\left\{a_{3}, a_{1}, a_{2}\right\}\left\{a_{4}, a_{5}\right\} & \\
& =3\left\{a_{1}, a_{2}, a_{3}\right\}\left\{a_{4}, a_{5}\right\} \bmod \gamma_{m+3}(R) R .
\end{aligned}
$$

Por outro lado, o lado esquerdo é igual a zero pela identidade de Jacobi. O resultado segue.

Lema 4.5.4 Seja $R$ uma álgebra de Poisson arbitrária sobre um corpo $K$, char $K \neq 2,3$. Então

$$
\left\{\gamma_{m}(R) R, R, R\right\} \subset \gamma_{m+2}(R) R, \quad m \geq 2 .
$$

Demonstração. Sejam $c \in \gamma_{m}(R)$ e $x, y, z \in R$. Considere o produto

$$
\{c x, y, z\}=c\{x, y, z\}+\{c, y\}\{x, z\}+\{c, z\}\{x, y\}+\{c, y, z\} x .
$$

Pelas propriedades dos comutadores de Lie, $\{c, y, z\} x \in \gamma_{m+2}(R) R$.

Claramente, $\{x, y, z\} \in \gamma_{3}(R)$, e como $c \in \gamma_{m}(R), m \geq 2$, podemos considerar que $c=\{a, b\}$, onde $a \in R, b \in \gamma_{m-1}(R)$. Aplicando o Lema 4.5.3, obtemos que $c\{x, y, z\} \in$ $\gamma_{m+2}(R) R$.

Considere os dois termos do meio restantes em (4.1)

$$
\begin{aligned}
&\{c, y\}\{x, z\}+\{c, z\}\{x, y\} \\
&=\{z, x\}\{y, c\}+\{y, x\}\{z, c\} \\
&=\{z y, x, c\}-z\{y, x, c\}-\{z, x, c\} y \in \gamma_{m+2}(R) R .
\end{aligned}
$$

Assim, $\{c x, y, z\} \in \gamma_{m+2}(R) R$, chegamos no resultado.

Provaremos a primeira afirmação do Teorema 4.5.1 como uma afirmação separada. 
Lema 4.5.5 Seja $R$ uma álgebra de Poisson arbitrária sobre um corpo $K$, char $K \neq 2,3$. Supondo que um dos inteiros $n, m \geq 1$ é ímpar, então

$$
\gamma_{n}(R) \cdot \gamma_{m}(R) \subseteq \gamma_{n+m-1}(R) \cdot R, \quad n, m \geq 1
$$

Demonstração. Vamos considerar que $n$ é ímpar e procederemos por indução sobre $n$. A base de indução $n=3$ é provada no Lema 4.5.3.

Agora, seja $n \geq 5$ ímpar. Considere $a \in \gamma_{n}(R)$ e $b \in \gamma_{m}(R)$. Como todos os produtos de Lie são combinações lineares de produtos de Lie normados à esquerda, sem perda de generalidade, consideramos que $a=\{c, x, y\}$, onde $c \in \gamma_{n-2}(R), x, y \in R$. Então

$$
\begin{aligned}
& a \cdot b=\{c, x, y\} b \\
& =\{c b, x, y\}-\{c, x\}\{b, y\}-\{c, y\}\{b, x\}-c\{b, x, y\}
\end{aligned}
$$

Considere o primeiro termo em (4.2). Pelo argumento de indução, $c b \in \gamma_{n+m-3}(R) R$, e pelo Lema 4.5.4, chegamos em $\{c b, x, y\} \in \gamma_{n+m-1}(R) R$.

Considere o último termo em (4.2). Claramente, $\{b, x, y\} \in \gamma_{m+2}(R)$, recordando que $c \in \gamma_{n-2}(R)$ e $n-2$ é ímpar. Pela hipótese de indução, $c\{b, x, y\} \in \gamma_{n+m-1}(R) R$.

Resta-nos considerar os dois termos do meio de (4.2).

$$
\begin{aligned}
& \{c, x\}\{b, y\}+\{c, y\}\{b, x\}=\{y, b\}\{x, c\}+\{x, b\}\{y, c\} \\
& =\{y x, b, c\}-\{y, b, c\} x-y\{x, b, c\} .
\end{aligned}
$$

Nos colchetes de Lie acima, $c \in \gamma_{n-2}(R), b \in \gamma_{m}(R)$ e os elementos restantes pertencem a $R$. Assim, todos os três produtos acima pertencem a $\gamma_{n+m-1}(R) R$. O lema está provado.

Provaremos a segunda afirmação do Teorema 4.5.1 como uma afirmação separada.

Lema 4.5.6 Seja $R$ uma álgebra de Poisson arbitrária sobre um corpo $K$, char $K \neq 2,3$. Para todos $x_{1}, \ldots, x_{n} \in R$, e todos $n, m \geq 2$, temos

$$
\left\{x_{1}, \ldots, x_{n}\right\}^{m} \in \gamma_{(n-1) m+1}(R) \cdot R \text {. }
$$


Demonstração. Caso 1: $n$ é ímpar. A afirmação segue pela sucessiva aplicação da afirmação 1 do Teorema 4.5.1, usamos este resultado abaixo regularmente sem menção especial.

Caso 2: $n$ é par e $m=2$. Denotaremos $a=\left\{x_{1}, \ldots, x_{n-1}\right\}, x=x_{n}$. Então $a \in \gamma_{n-1}(R)$ e $\left\{x_{1}, \ldots, x_{n}\right\}=\{a, x\}$. Considere

$$
\left\{\left\{x^{2}, a\right\}, a\right\}=\{2 x\{x, a\}, a\}=2\{x, a\}\{x, a\}+2 x\{x, a, a\} .
$$

Usando as propriedades dos comutadores de Lie, chegamos na inclusão desejada:

$$
\{x, a\}^{2}=\frac{1}{2}\left\{\left\{x^{2}, a\right\}, a\right\}-x\{x, a, a\} \in \gamma_{2 n-1}(R) R .
$$

Caso 3: $n$ é par e $m=2 q, q>1$. Aplicamos o Caso 2 e o Caso 1:

$$
\begin{aligned}
\left\{x_{1}, \ldots, x_{n}\right\}^{2 q}=\left(\left\{x_{1}, \ldots, x_{n}\right\}^{2}\right)^{q} & \in\left(\gamma_{2 n-1}(R) R\right)^{q} \\
& \left.=\left(\gamma_{2 n-1}(R)\right)^{q} R \subset \gamma_{(2 n-2) q+1}(R)\right) R=\gamma_{(n-1) m+1}(R) R .
\end{aligned}
$$

Caso 4: $n$ é par e $m=2 q+1, q \geq 1$. Usamos os casos anteriores.

$$
\begin{aligned}
\left\{x_{1}, \ldots, x_{n}\right\}^{m}=\left\{x_{1}, \ldots,\right. & \left.x_{n}\right\}^{2 q}\left\{x_{1}, \ldots, x_{n}\right\} \\
& \in \gamma_{(n-1) 2 q+1}(R) \gamma_{n}(R) \subset \gamma_{(n-1)(m-1)+n}(R) R=\gamma_{(n-1) m+1}(R) R .
\end{aligned}
$$

O lema está provado.

O seguinte lema é um análogo dos respectivos fatos para álgebras associativas, veja [25] e [19]. Ele é mais fraco do que a Afirmação 1 do Teorema 4.5.1, mas ele é válido para uma característica arbitrária.

Lema 4.5.7 Seja $R$ uma álgebra de Poisson arbitrária sobre um corpo arbitrário $K$. Então

$$
\gamma_{m}(R) \gamma_{n}(R) \subset \gamma_{m+n-2}(R) R, \quad n, m \geq 2
$$

Demonstração. Procedemos por indução sobre $n \geq 2$ para algum $m \geq 2$ arbitrário. No caso $n=2$ não há o que provar. 
Assumiremos que $n>2$. Para estudar $\gamma_{m}(R) \gamma_{n}(R)$ é suficiente considerar os produtos $u=\{r, a\}\left\{x, y, z_{1}, \ldots, z_{n-2}\right\}$, onde $r \in \gamma_{m-1}(R)$ e todos os outros elementos pertencentes a $R$. Considere um produto $w=\left\{a\{x, y\}, r, z_{1}, \ldots, z_{n-2}\right\}$, comutando o comprimento de Lie total, ganhamos $w \in \gamma_{n+m-2}(R)$. Por outro lado

$$
\begin{aligned}
w & =\left\{a\{x, y\}, r, z_{1}, \ldots, z_{n-2}\right\}=\{a, r\}\left\{x, y, z_{1}, \ldots, z_{n-2}\right\} \\
& +\sum_{s=1}^{n-2} \sum_{i, j}\left\{a, r, z_{i_{1}}, \ldots, z_{i_{s}}\right\}\left\{x, y, z_{j_{1}}, \ldots, z_{j_{n-2-s}}\right\} \\
& +\sum_{s=0}^{n-2} \sum_{i, j}\left\{a, z_{i_{1}}, \ldots, z_{i_{s}}\right\}\left\{x, y, r, z_{j_{1}}, \ldots, z_{j_{n-2-s}}\right\}
\end{aligned}
$$

onde as somas acima correspondem a todas as partições do conjunto $\{1, \ldots, n-2\}$ em dois subconjuntos de índices tais que $i_{1}<\cdots<i_{s}, j_{1}<\cdots<j_{n-2-s}$. O primeiro termo nos dá o produto desejado $-u$. Os produtos do primeiro par de somas pertencem a $\gamma_{m+s}(R) \gamma_{n-s}(R) \in \gamma_{n+m-2}(R) R$ pela hipótese de indução pois $s>1$. Os produtos do segundo par de somas pertencem a $\gamma_{s+1}(R) \gamma_{n+m-s-1}(R) \in \gamma_{n+m-2}(R) R$ pela hipótese de indução pois $s+1<n$. Assim, o lema está provado.

\subsection{A classe de Lie nilpotência}

Agora nós demonstramos a equivalência dos itens 1 e 3 do nosso Teorema 3.3.2.

Teorema 4.6.1 Seja L uma álgebra de Lie sobre um corpo $K$ de característica positiva $p>3$, tal que $\mathbf{s}(L)$ é Lie nilpotente. Então a classe de Lie nilpotência de $\mathbf{s}(L)$ é igual a

$$
1+(p-1) \sum_{n \geq 1} n \cdot \operatorname{dim}\left(\gamma_{n+1}(L) / \gamma_{n+2}(L)\right)
$$

Demonstração. Pelo Teorema 3.3.1, $L$ é nilpotente e $L^{2}$ tem dimensão finita. Denotando $d_{i}=\operatorname{dim} \gamma_{i+1}(L) / \gamma_{i+2}(L), i \geq 1$, pelas condições, eles são finitos. Seja $d_{s} \neq 0$ mas $d_{s+1}=d_{s+2}=\ldots 0$, para algum $s$. Seja $\left\{e_{i j} \mid 1 \leq j \leq d_{i}\right\}, i \geq 1$ uma base para $\gamma_{i+1}(L)$ módulo $\gamma_{i+2}(L), 1 \leq i \leq s$. Considere

$$
0 \neq v=e_{11}^{p-1} \cdots e_{1 d_{1}}^{p-1} e_{21}^{p-1} \cdots e_{2 d_{2}}^{p-1} \cdots e_{s 1}^{p-1} \cdots e_{s d_{s}}^{p-1}
$$


Observe que os elementos da base acima podem ser escolhidos como comutadores, isto é $e_{i j}=\left\{y_{1}, \ldots, y_{i+1}\right\}$, onde $y_{j} \in R$. Pela Afirmação 2 do Teorema 4.5.1,

$$
e_{i j}^{p-1} \in \gamma_{i(p-1)+1}(R) \cdot R, \quad j=1, \ldots d_{i},
$$

para todos os fatores acima. Uma vez que todos os números $i(p-1)+1$ são ímpares, podemos usar a Afirmação 1 do Teorema 4.5.1 e obtemos

$$
e_{i 1}^{p-1} \cdots e_{i d_{i}}^{p-1} \in \gamma_{i d_{i}(p-1)+1}(R) \cdot R
$$

A seguir, consideramos o produto total (4.3):

$$
0 \neq v \in \gamma_{N+1}(R) \cdot R \text {, onde } N=(p-1) \sum_{i=1}^{s} i d_{i} .
$$

Assim, $\gamma_{N+1}(R) \not \equiv\{0\}$. Por outro lado, $\gamma_{N+2}(R) \subset R^{(N+1)} \equiv\{0\}$ pela relação (3.2) e pelo Corolário 4.3.3. Então, $\mathbf{s}(L)$ é Lie nilpotente de classe $N+1$.

Agora, podemos estabelecer uma cota inferior para a classe de Lie nilpotência para características $p=2,3$.

Teorema 4.6.2 Seja L uma álgebra de Lie não abeliana sobre um corpo de características positiva, tal que $\mathbf{s}(L)$ é Lie nilpotente. Então a classe de Lie nilpotência de $\mathbf{s}(L)$ é limitada acima pela classe de Lie nilpotência forte e limitada abaixo pelo seguinte número

$$
2+(p-1) \sum_{n \geq 2}(n-1) \cdot \operatorname{dim}\left(\gamma_{n+1}(L) / \gamma_{n+2}(L)\right)
$$

Demonstração. Sejam $e_{i j} \in \gamma_{i+1}(L)$ elementos da base escolhidos como na prova do Teorema 4.5.1. Agora, usando o Lema 4.5.7, chegamos em

$$
e_{i j}^{p-1} \in \gamma_{(p-1)(i-1)+2}(R) \cdot R, \quad 1 \leq j \leq d_{i}, 1 \leq i \leq s
$$


Uma vez que $L$ é não abeliana, temos pelo menos um vetor $e_{11} \in \gamma_{2}(R)$, e o produto (4.3) é não trivial. Aplicando o Lema 4.5.7 para o produto todo (4.3), ganhamos

$$
0 \neq v \in \gamma_{M}(R) \cdot R, \text { onde } M=(p-1) \sum_{i=1}^{s}(i-1) d_{i}+2
$$

Assim, $\gamma_{M}(R) \neq 0$. Portanto, $M$ é um limite inferior para a classe de nilpotência de $\mathbf{s}(L)$. 


\section{Capítulo 5}

\section{Lie solubilidade. Demonstrações}

Neste capítulo demonstramos o nosso segundo resultado principal sobre solubilidade da álgebra Poisson simétrica truncada, isto é, nós demonstramos o teorema a seguir.

Teorema 3.4.1. Seja L uma álgebra de Lie sobre um corpo de característica $p \geq 3$. Considere sua álgebra de Poisson simétrica truncada $\mathbf{s}(L)$. As seguintes condições são equivalentes:

1. $\mathbf{s}(L)$ é Lie solúvel forte;

2. $\mathbf{s}(L)$ é Lie solúvel;

3. L é solúvel e $\operatorname{dim} L^{2}<\infty$.

No caso $p=2$ as condições 1 e 3 são equivalentes também.

\subsection{Lie solubilidade da álgebra simétrica truncada}

Nesta seção provaremos o Teorema 3.4.1 todo no caso $p \neq 2$.

Primeiro, mostraremos que 3 implica 1 para caraterística qualquer positiva. Vamos supor que $L$ é solúvel e $\operatorname{dim} L^{2}<\infty$. Como $L$ é solúvel, temos uma sequência decrescente 
de ideais

$$
L=\delta_{0}(L) \supset \delta_{1}(L)=L^{2} \supset \delta_{2}(L) \supset \ldots \supset \delta_{k-1}(L) \supset \delta_{k}(L)=0 .
$$

Para ver que $\mathbf{s}(L)$ é Lie solúvel forte, iremos proceder por indução sobre o comprimento de solubilidade $k$. A base de indução $k=1$ é trivial, uma vez que $L$ é abeliana e daí $\mathbf{s}(L)$ será também.

Vamos supor que $k>1$. Seja $\bar{L}=L / \delta_{k-1}(L)$ e considere a aplicação induzida $\varphi: \mathbf{s}(L) \rightarrow \mathbf{s}(\bar{L})$. Portanto, pela hipótese de indução, $\mathbf{s}(\bar{L})$ é Lie solúvel forte, então existe $m$ tal que $\overline{\widetilde{\delta}_{m}(\mathbf{s}(L))}=\overline{0}$, isto implica que $\widetilde{\delta}_{m}(\mathbf{s}(L)) \subseteq J=\mathbf{s}(L) \delta_{k-1}(L)$, que é o núcleo de $\varphi$. Escolhemos $\left\{a_{i} \in L \backslash \delta_{k-1}(L) \mid i \in I\right\}$ uma base do espaço quociente $L / \delta_{k-1}(L)$ e $\left\{b_{1}, \ldots, b_{l}\right\}$ uma base finita de $\delta_{k-1}(L)$. Agora, seja $w \in J$ um monômio da PBW-base de $J$, então $w$ é da forma $w=a^{\alpha} b^{\beta}$, onde

$$
\begin{aligned}
& a^{\alpha}=a_{1}^{\alpha_{1}} a_{2}^{\alpha_{2}} \cdots a_{n}^{\alpha_{n}}, \quad 0 \leq \alpha_{i}<p, \\
& b^{\beta}=b_{1}^{\beta_{1}} b_{2}^{\beta_{2}} \cdots b_{l}^{\beta_{l}} \in \mathbf{s}\left(\delta_{k-1}(L)\right), \quad 0 \leq \beta_{j} \leq p-1,
\end{aligned}
$$

onde pelo menos um $\beta_{j}$ é não nulo. Denotaremos por $|\alpha|=\alpha_{1}+\alpha_{2}+\cdots+\alpha_{n}$ e por $|\beta|=\beta_{1}+\beta_{2}+\cdots+\beta_{l}$. Observe que $|\beta|>1$. Então considere $w_{1}, w_{2}$ como descrito acima, e considere o produto deles $\left\{w_{1}, w_{2}\right\}=\sum u_{j} \in \widetilde{\delta}_{1}(\mathbf{s}(L))$. Pela regra de Leibnitz, este produto é uma soma de produtos que contêm fatores do tipo $\left\{a_{i}, a_{j}\right\}$ ou

$$
\left\{a_{i}, b_{j}\right\}=\sum_{t=1}^{l} \lambda_{t} b_{t} \in \delta_{k-1}(L), \quad \lambda_{t} \in K
$$

(uma vez que $\delta_{k-1}(L)$ é um ideal de $L$ ), observe que $\left\{b_{i}, b_{j}\right\}=0$ (pois $\delta_{k-1}(L)$ é abeliano). Em todos os casos obtemos monômios em que $\left|\beta^{\prime \prime}\right| \geq|\beta|+\left|\beta^{\prime}\right| \geq 2$, isto é, em $\left\{w_{1}, w_{2}\right\}$ existem pelo menos dois $b_{j}^{\prime}$ s. Como $\widetilde{\delta}_{m}(\mathbf{s}(L))$ contém pelo menos um $b_{j}$ concluímos que $\widetilde{\delta}_{m+N}(\mathbf{s}(L))$ contém pelo menos $2^{N}$ de tais elementos. Como $2^{N}>p^{l}$, onde pela hipótese $\operatorname{dim} L^{2}=l<\infty$, temos que $\widetilde{\delta}_{m+N}(\mathbf{s}(L))=0$, como desejado.

A implicação 1 para 2 segue de $\delta_{n}(\mathbf{s}(L)) \subseteq \widetilde{\delta}_{n}(\mathbf{s}(L))$, por (3.3).

Agora provaremos que 2 implica 3 no caso $p=2$. Assumiremos que $\mathbf{s}(L)$ é Lie solúvel. Como $L \subset \mathbf{s}(L)$, claramente $L$ é solúvel. Assim, resta-nos provar que $L^{2}$ tem dimensão finita. Para provar este fato, nós podemos assumir que $K$ é algebricamente fechado. Como $\mathbf{s}(L)$ satisfaz uma identidade Poisson multilinear não-trivial, pelo Lema $4.1 .1 \mathrm{e}$ Teorema 4.1.4, para algum número $M$ temos $\Delta(L)=\Delta_{M}(L)$ e $L / \Delta(L)$ tem dimensão 
finita. Indicaremos $\Delta(L)$ apenas por $\Delta$. Usando o Teorema 4.1.2 para a aplicação bilinear $\Delta \times \Delta \rightarrow \Delta$, onde $(u, v) \mapsto[u, v]$, para todo $u, v \in \Delta$ nós ganhamos também que $\operatorname{dim} \Delta^{2} \leq M^{2}$ tem dimensão finita. Dessa forma, é suficiente provar que $L=\Delta$. Para fazer isto, vamos supor que $L \neq \Delta$. Argumentando como na prova do Teorema 3.3.1, assumiremos que $\Delta$ é abeliano. De fato, considerando a álgebra quociente $\bar{L}=L / \Delta^{2}$, por $\operatorname{dim} \Delta^{2}$ ser finita, pelo Lema 4.1.3, temos $\Delta(\bar{L})=\Delta / \Delta^{2}$. Portanto, $\Delta(\bar{L})$ é abeliano e $\bar{L} \neq \Delta(\bar{L})$. Substituiremos $L$ por $\bar{L}$.

O próximo resultado também é válido para o caso $p=2$.

Lema 5.1.1 Suponha que L é uma álgebra de Lie sobre um corpo $K$ de característica positiva $p, x \in L \backslash \Delta$ e $\Delta$ é um ideal abeliano. Seja $\mathbf{s}(L)$ (ou a álgebra simétrica $S(L)$ sobre um corpo arbitrário) uma álgebra de Lie solúvel de comprimento s. Então ad $x$ age algebricamente sobre $\Delta$, de grau limitado por

$$
M(s)=(s+1) 2^{s}+s .
$$

Demonstração. Como $\mathbf{s}(L)$ é Lie solúvel, ela satisfaz a identidade $\delta_{s}\left(x_{1}, x_{2}, \ldots, x_{2^{s}}\right) \equiv 0$. Seja $x \in L \backslash \Delta$ e $0 \neq v \in \Delta$. Queremos provar que o espaço $V=\left\langle(\operatorname{ad} x)^{i} v\right| i=$ $0,1,2, \ldots\rangle_{K}=\left\langle v_{0}, v_{1}, v_{2}, \ldots\right\rangle_{K}$ é de dimensão finita, mais ainda, que para o tal $M$, os elementos

$$
v_{0}=v, v_{1}=(\operatorname{ad} x)^{1} v, v_{2}=(\operatorname{ad} x)^{2} v, \ldots, v_{M}=(\operatorname{ad} x)^{M} v,
$$

são linearmente dependentes. Vamos supor o contrário, que $\left\{(\operatorname{ad} x)^{i} v \mid i=0,1,2, \ldots, M\right\}$ é um conjunto linearmente independente. Mostraremos que, escolhendo $N$ suficientemente grande $\delta_{s}\left(x v_{N}, x v_{2 N}, \ldots, x v_{2^{s} N}\right)$ é um elemento não nulo de $\mathbf{s}(L)$, chegando assim numa contradição com o fato que $\delta_{s}\left(x_{1}, x_{2}, \ldots, x_{2^{s}}\right) \equiv 0$ em $\mathbf{s}(L)$.

Vamos comparar os elementos do mesmo comprimento da forma $u=x v_{\alpha_{1}} v_{\alpha_{2}} \ldots$ $v_{\alpha_{k}}, w=x v_{\beta_{1}} v_{\beta_{2}} \cdots v_{\beta_{k}}$ lexicograficamente começando com os índices maiores. Ou seja, assumiremos que $\alpha_{1} \leq \alpha_{2} \leq \cdots \leq \alpha_{k}, \beta_{1} \leq \beta_{2} \leq \cdots \leq \beta_{k}$. Então $u<w$ se, e somente se, $\alpha_{k}<\beta_{k}$, ou $\alpha_{k}=\beta_{k}$, mas $\alpha_{k-1}<\beta_{k-1}$, etc.

Note que, para $n=1$, por $\Delta$ ser abeliano e usando a regra de Leibnitz, pela substi- 
tuição $x_{1}=x v_{N}, x_{2}=x v_{2 N}$ temos

$$
\begin{aligned}
\delta_{1}\left(x_{1}, x_{2}\right) & =\left\{x_{1}, x_{2}\right\}=\left\{x v_{N}, x v_{2 N}\right\} \\
& =x\left(v_{N} v_{2 N+1}-v_{N+1} v_{2 N}\right)
\end{aligned}
$$

onde os elementos $x v_{N} v_{2 N+1}$ e $x v_{N+1} v_{2 N}$ são não nulos e diferentes.

Para $n=2$, fazendo o mesmo processo, i.é., fazendo as substituições $x_{1}=x v_{N}, x_{2}=$ $x v_{2 N}, x_{3}=x v_{3 N}$ e $x_{4}=x v_{4 N}$, obtemos

$$
\begin{aligned}
\delta_{2}\left(x v_{N}, x v_{2 N}, x v_{3 N}, x v_{4 N}\right)= & \left\{\delta_{1}\left(x v_{N}, x v_{2 N}\right), \delta_{1}\left(x v_{3 N}, x v_{4 N}\right)\right\} \\
= & \left\{x v_{N} v_{2 N+1}-x v_{N+1} v_{2 N}, x v_{3 N} v_{4 N+1}-x v_{3 N+1} v_{4 N}\right\} \\
= & \left\{x v_{N} v_{2 N+1}-x v_{N+1} v_{2 N}, x v_{3 N} v_{4 N+1}\right\} \\
& -\left\{x v_{N} v_{2 N+1}-x v_{N+1} v_{2 N}, x v_{3 N+1} v_{4 N}\right\} \\
= & x\left(v_{N} v_{2 N+1}-v_{N+1} v_{2 N}\right)\left(v_{3 N} v_{4 N+2}-v_{3 N+2} v_{4 N}\right) \\
& +x\left(v_{N+2} v_{2 N}-v_{N} v_{2 N+2}\right)\left(v_{3 N} v_{4 N+1}-v_{3 N+1} v_{4 N}\right)
\end{aligned}
$$

onde o elemento $x v_{N} v_{2 N+1} v_{3 N} v_{4 N+2}$ é o único maximal com respeito à ordem lexicográfica.

Semelhantemente, para qualquer $k \geq 1$, fazendo a mesma avaliação $x_{i}=x v_{i N}$, para todos $i=1,2, \ldots, 2^{k}$ em $\delta_{k}\left(x_{1}, x_{2}, \ldots, x_{2^{k}}\right)$, chegamos na soma

$$
\begin{aligned}
& \sum_{\epsilon_{j}} \theta_{\epsilon} x v_{N+\varepsilon_{1}} v_{2 N+\varepsilon_{2}} \cdots v_{2^{k} N+\varepsilon_{2^{k}}}, \quad \theta_{\epsilon} \in \mathbb{F}_{p} ; \quad \text { para alguns } \epsilon_{j} \text { satisfazendo: } \\
& \epsilon_{1}+\epsilon_{2}+\cdots+\epsilon_{2^{k}}=2^{k}-1, \quad 0 \leq \varepsilon_{i} \leq k, \quad i=1,2, \ldots, 2^{k} .
\end{aligned}
$$

A soma contém um único elemento maior em termos da ordem lexicográfica, i.é., ele tem o coeficiente $\theta_{\epsilon}$ igual a 1 , que é não nulo em $\mathbf{s}(L)$ uma vez que ele é um elemento da base.

De fato, para $k$ temos um único elemento maximal (5.1) dado pela tupla $\left(\alpha_{1}, \alpha_{2}, \ldots\right.$, $\alpha_{2^{k}}$ ), então o único elemento maximal para $k+1$ na expansão (5.1) é dado pela tupla

$$
\left(\alpha_{1}, \ldots, \alpha_{2^{k}}, \alpha_{1}, \ldots, \alpha_{2^{k}-1}, \alpha_{2^{k}}+1\right) \text {. }
$$

Agora, considere $k=s$ e fixemos $N=s+1$ para garantir que os índices em (5.1) não se sobreponham. Assim, os fatores em cada produto $x v_{N+\varepsilon_{1}} v_{2 N+\varepsilon_{2}} \cdots v_{2^{s} N+\varepsilon_{2^{s}}}$ são 
diferentes. Porém, isto contradiz ao fato que $\delta_{s}\left(x_{1}, x_{2}, \ldots, x_{2^{s}}\right) \equiv 0$ em $\mathbf{s}(L)$. Portanto, a aplicacão ad $x$ age algebricamente sobre $\Delta$. O índice mais extremo que aparece em (5.1) é limitado por $M(s)=(s+1) 2^{s}+s$.

Vamos fixar $x \in L / \Delta$ e denotaremos por $V^{v}$ um subespaço de $\Delta$ gerado por $v$. Ou seja: $V^{v}=\left\langle(\operatorname{ad} x)^{i} v \mid i \geq 0\right\rangle$. Então pelo Lema 5.1.1, $\operatorname{dim} V^{v} \leq M(s)$ para todo $v \in \Delta$. Note que $V^{v}$ tem uma decomposição de Jordan

$$
V^{v}=\bigoplus_{i} V_{\left(\lambda_{i}\right)}^{v}, \quad V_{\left(\lambda_{i}\right)}^{v}=\left\{y \in V^{v} \mid\left(\operatorname{ad} x-\lambda_{i} E\right)^{m_{i}} y=0\right\}
$$

onde $m_{i}$ é o número minimal positivo. Vamos juntar tais decomposições para diferentes $v \in \Delta$ para obtermos $\Delta=\oplus_{i \in I} V_{\left(\lambda_{i}\right)}$.

Para qualquer subsoma finita $\oplus_{i=1}^{k} V_{\left(\lambda_{i}\right)}$ o grau do polinômio para a ação $(\operatorname{ad} x)$ é $m_{1}+m_{2}+\cdots+m_{k}$. Pelo Lema 5.1.1, $m_{1}+m_{2}+\cdots+m_{k} \leq M(s)$. Portanto, temos uma soma finita:

$$
\Delta=\bigoplus_{i=1}^{k} V_{\left(\lambda_{i}\right)}, \quad k \leq M(s), \quad m_{i} \leq M(s), \quad i=1, \ldots, k .
$$

Também, pelas propriedades da decomposição de Jordan, $\operatorname{dim} V_{\left(\lambda_{i}\right)} \leq \operatorname{dim} V_{\lambda_{i}} \cdot M(s)$, $i=1, \ldots, k$.

Como $L=\langle x\rangle \oplus \Delta$, onde $\operatorname{dim}\{x, \Delta\}=\infty$, devemos ter $\operatorname{dim}\left\{x, V_{\left(\lambda_{i}\right)}\right\}=\infty$ para algum $i=1, \ldots, k$. Denote $\lambda=\lambda_{i}$ e nossa prova será reduzida ao caso com uma componente só: $L=\langle x\rangle_{K} \oplus V_{(\lambda)}$.

Em nossa prova, consideramos dois casos: $\lambda=0$ e $\lambda \neq 0$.

Considere o caso $\lambda=0$.

Neste caso, temos $V_{(0)}^{v}=\left\{y \mid(\operatorname{ad} x)^{m} y=0\right\}$, onde $m$ é minimal. Podemos assumir que $(\operatorname{ad} x)^{2}=0$. Esta redução é feita como no caso de Lie nilpotência. Logo abaixo, nós seguiremos os argumentos de [42].

Proposição 5.1.2 Seja L uma álgebra de Lie sobre um corpo $K$ de característica ímpar tal que

1. $\Delta=\Delta(L)$ é abeliano; 
2. $L=\langle x\rangle \oplus \Delta,(\operatorname{ad} x)^{2} \Delta=0, \operatorname{dim}\{x, \Delta\}=\infty$.

Então $\mathbf{s}(L)$ não é Lie solúvel.

Adotaremos a seguinte notação. Vamos denotar $R=\mathbf{s}(L)$ e $a^{\prime}=\{x, a\}$, para qualquer $a \in \mathbf{s}(\Delta)$. Denotaremos também por $\langle x\rangle$ o conjunto $\left\{x^{i} \mid 0 \leq i \leq p-1\right\}$. Neste caso, $R=\langle x\rangle \mathbf{s}(\Delta)$. Seja $S=\left\{a \in \mathbf{s}(\Delta) \mid a^{\prime \prime}=0\right\}$. Note que $S$ é um subespaço linear de $\mathbf{s}(\Delta)$, mas não necessariamente um subanel. Porém, iremos fazer a seguinte afirmação.

Lema 5.1.3 Se $a, b \in S$, então $a^{\prime} b \in S$.

Demonstração. Como $a, b \in S$, então $a^{\prime \prime}=\{x,\{x, a\}\}=0$ e $b^{\prime \prime}=\{x,\{x, b\}\}=0$. Pela regra de Leibnitz, segue que $\left(a^{\prime} b\right)^{\prime \prime}=\left\{x,\left\{x, a^{\prime} b\right\}\right\}=\left\{x, a^{\prime} b^{\prime}\right\}=0$.

Em particular, $a^{\prime} b+a b^{\prime} \in S$ sempre que $a, b \in S$. Assim, temos o próximo lema.

Lema 5.1.4 Sejam $a, b \in S$. Então para todo $i>0$ e $j \geq 0$ as seguintes identidades valem.

1. $\left\{x^{i}, a\right\}=i x^{i-1} a^{\prime}$;

2. $\left\{x^{i} a, x^{j} b\right\}=x^{i+j-1}\left(i a b^{\prime}-j a^{\prime} b\right)$.

Demonstração. Para a prova de 1 usaremos indução sobre $i$.

Para $i=1$, temos a definição $\{x, a\}=a^{\prime}$. Agora, suponhamos que a afirmação seja válida para $i>1$, iremos ver que é válida também para $i+1$. Usando a regra de Leibnitz, temos

$$
\begin{aligned}
\left\{x^{i+1}, a\right\} & =\left\{x \cdot x^{i}, a\right\} \\
& =x\left\{x^{i}, a\right\}+x^{i}\{x, a\} \\
& =x i x^{i-1} a^{\prime}+x^{i} a^{\prime} \\
& =(i+1) x^{i} a^{\prime} .
\end{aligned}
$$


Portanto, 1 é válido para todo $i>0$. Para 2, pela parte 1 temos $\left\{a, x^{j}\right\}=-j x^{j-1} a^{\prime}$. Portanto $\left\{x^{i} a, x^{j}\right\}=x^{i}\left\{a, x^{j}\right\}=-j x^{i+j-1} a^{\prime}$ e $\left\{x^{i} a, b\right\}=a\left\{x^{i}, b\right\}=i x^{i-1} a b^{\prime}$ pela parte 1 . Dessa forma,

$$
\begin{aligned}
\left\{x^{i} a, x^{j} b\right\} & =x^{j} i x^{i-1} a b^{\prime}-j x^{i+j-1} a^{\prime} b \\
& =x^{i+j-1}\left(i a b^{\prime}-j a^{\prime} b\right) .
\end{aligned}
$$

Lema 5.1.5 Sejam $a, b \in S$ tais que $\langle x\rangle a,\langle x\rangle b \subseteq \delta_{n-1}(R)$, então

$$
\langle x\rangle\left(a^{\prime} b+a b^{\prime}\right) \subseteq \delta_{n}(R)
$$

Demonstração. Para $1 \leq j \leq p-1$ temos pelo Lema 5.1 .4 que

$$
-j x^{j-1} a^{\prime} b \in \delta_{n-1}(R)^{\prime}=\delta_{n}(R)
$$

Portanto $x^{i} a^{\prime} b \in \delta_{n}(R)$, e analogamente $x^{i} a b^{\prime} \in \delta_{n}(R)$, para $i=0,1, \ldots, p-2$. Restanos considerar o caso $i=p-1$. Pelo Lema 5.1.4,

$$
\begin{aligned}
x^{p-1}\left(a b^{\prime}+a^{\prime} b\right) & =x^{p-1}\left(1 a b^{\prime}-(p-1) a^{\prime} b\right) \\
& =\left\{x^{1} a, x^{p-1} b\right\} \in \delta_{n}(R) .
\end{aligned}
$$

Isto completa a prova.

Vamos munir $S$ com uma nova operação bilinear dada por $(a, b) \mapsto a \diamond b=a^{\prime} b+a b^{\prime}$.

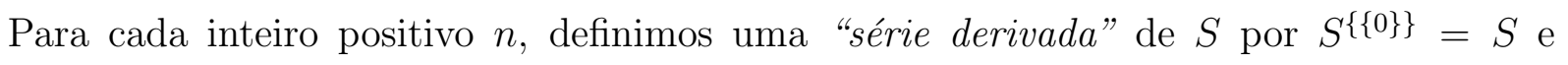
$S^{\{\{n\}\}}=S^{\{\{n-1\}\}} \diamond S^{\{\{n-1\}\}}$, o espaço vetorial gerado pelos elementos $a \diamond b$ onde $a, b \in$ $S^{\{\{n-1\}\}}$ e $n \geq 1$.

Lema 5.1.6 Para todo $n \geq 0$ temos

$$
\langle x\rangle S^{\{\{n\}\}} \subseteq \delta_{n}(R)
$$

Demonstração. Segue do Lema 5.1.5 por indução sobre $n$. 
Para todo $a_{1}, a_{2}, \ldots, a_{l} \in S$, definiremos o novo produto:

$$
\left\{\left\{a_{1}, a_{2}, \ldots, a_{l}\right\}\right\}=a_{1}^{\prime} a_{2}^{\prime} \cdots a_{l-1}^{\prime} a_{l}+a_{1}^{\prime} a_{2}^{\prime} \cdots a_{l-2}^{\prime} a_{l-1} a_{l}^{\prime}+\cdots+a_{1} a_{2}^{\prime} \cdots a_{l}^{\prime} .
$$

Então, com respeito a esse produto obtemos o seguinte resultado.

Lema 5.1.7 Para todo $n$ é válido que

$$
\left\{\left\{\left\{a_{1}, a_{2}, \ldots, a_{2^{n}}\right\}\right\} \mid a_{1}, a_{2}, \ldots, a_{2^{n}} \in S\right\} \subseteq S^{\{\{n\}\}}
$$

Demonstração. Faremos a prova por indução sobre $n$. Para $n=0$ temos que $\left\{\left\{a_{1}\right\}\right\} \in$ $S=S^{\{\{0\}\}}$ e para $n=1,\left\{\left\{a_{1}, a_{2}\right\}\right\}=a_{1} \diamond a_{2} \in S \diamond S=S^{\{\{1\}\}}$.

Note que, como $a_{1}, a_{2}, \ldots, a_{2^{n}} \in S$, temos $\left\{\left\{a_{1}, a_{2}, \ldots, a_{2^{n}}\right\}\right\}^{\prime}=2^{n} a_{1}^{\prime} a_{2}^{\prime} \cdots a_{2^{n}}^{\prime}$. Portanto,

$$
\begin{aligned}
& \left\{\left\{a_{1}, a_{2}, \ldots, a_{2^{n}}\right\}\right\} \diamond\left\{\left\{b_{1}, b_{2}, \ldots, b_{2^{n}}\right\}\right\} \\
& =2^{n}\left(a_{1}^{\prime} a_{2}^{\prime} \cdots a_{2^{n}}^{\prime}\left\{\left\{b_{1}, b_{2}, \ldots, b_{2^{n}}\right\}\right\}+\left\{\left\{a_{1}, a_{2}, \ldots, a_{2^{n}}\right\}\right\} b_{1}^{\prime} b_{2}^{\prime} \cdots b_{2^{n}}^{\prime}\right) ; \\
& =2^{n}\left\{\left\{a_{1}, a_{2}, \ldots, a_{2^{n}}, b_{1}, b_{2}, \ldots, b_{2^{n}}\right\}\right\} .
\end{aligned}
$$

Por indução, segue que

$$
\left\{\left\{a_{1}, a_{2}, \ldots, a_{2^{n}}\right\}\right\},\left\{\left\{b_{1}, b_{2}, \ldots, b_{2^{n}}\right\}\right\} \in S^{\{\{n\}} .
$$

Recorde que estamos assumindo $p>2$, assim chegamos que

$$
2^{n}\left\{\left\{a_{1}, a_{2}, \ldots, a_{2^{n}}, b_{1}, b_{2}, \ldots, b_{2^{n}}\right\}\right\} \in S^{\{\{n\}\}} \diamond S^{\{\{n\}\}}=S^{\{\{n+1\}\}} .
$$

Usando o Lema 5.1.6 e o Lema 5.1.7 obtemos o próximo resultado.

Lema 5.1.8 Para todo $a_{1}, a_{2}, \ldots, a_{2^{n}} \in S$ vale que

$$
\langle x\rangle\left\{\left\{a_{1}, a_{2}, \ldots, a_{2^{n}}\right\}\right\} \subseteq \delta_{n}(R),
$$

para qualquer $n \geq 0$. 
Demonstração. Como $x \notin \Delta, L^{2}=\{x, \Delta\}$ tem dimensão infinita, escolhemos uma sequência $\left\{\left.a_{n}\right|_{n \geq 1}\right\} \subseteq \Delta$ tal que $\left\{a_{n}^{\prime} \mid n \geq 1\right\}$ é um conjunto linearmente independente. Afirmamos que $\left\{a_{n} \mid n \geq 1\right\}$ é linearmente independente módulo $L^{2}$. Com efeito, se $\sum_{n=1}^{k} \lambda_{n} a_{n} \in L^{2}$ para algum $\lambda_{n} \in K$, então

$$
\sum_{n=1}^{k} \lambda_{n} a_{n}^{\prime}=\sum_{n=1}^{k} \lambda_{n}\left\{x, a_{n}\right\}=\left\{x, \sum_{n=1}^{k} \lambda_{n} a_{n}\right\} \in\left\{x, L^{2}\right\}=\{x, x, \Delta\}=0
$$

uma vez que $(\operatorname{ad} x)^{2}=0$, portanto $\lambda_{n}=0$ para todo $n$. Isto mostra que $\left\{a_{n} \mid n \geq\right.$ $1\} \cup\left\{a_{n}^{\prime} \mid n \geq 1\right\}$ é um conjunto linearmente independente. Como $\mathbf{s}(\Delta)$ é um anel de polinômios truncados, os monômios que aparecem em $\left\{\left\{a_{1}, a_{2}, \ldots, a_{l}\right\}\right\}$ são linearmente independente em $\mathbf{s}(\Delta) \subseteq R$. Portanto, $\left\{\left\{a_{1}, a_{2}, \ldots, a_{2^{n}}\right\}\right\} \neq 0$ para todo $n$, e assim $\delta_{n}(R) \neq 0$ para todo $n$ pelo Lema 5.1.7. Uma contradição.

Agora, os lemas anteriores completam a prova da Proposição 5.1.2.

\section{Considere o caso $\lambda \neq 0$.}

Temos o autoespaço $V_{\lambda}$, o qual está contido no bloco de Jordan $V_{(\lambda)}$ (veja Lema 5.1.1). Observe que $\operatorname{dim} V_{(\lambda)} \leq \operatorname{dim} V_{\lambda} \cdot M$, onde $M$ é o grau de algebricidade da ação de $x$ sobre $\Delta$. Como $\operatorname{dim} V_{(\lambda)}=\infty$ então $\operatorname{dim} V_{\lambda}=\infty$. Uma vez que as igualdades $\langle x\rangle=\left\langle\frac{1}{\lambda} x\right\rangle \mathrm{e}$ $\operatorname{ad}\left(\frac{1}{\lambda} x\right)(y)=\frac{1}{\lambda} \operatorname{ad} x(y)=y$ para todo $y \in V_{\lambda}$, são válidas, podemos considerar $\lambda=1$, e nosso caso é reduzido ao seguinte resultado.

Proposição 5.1.9 Seja L uma álgebra de Lie de característica ímpar tal que

1. $\Delta=\Delta(L)$ é abeliano;

2. $L=\langle x\rangle \oplus \Delta,\{x, v\}=v$, para todo $v \in \Delta e \operatorname{dim} \Delta=\infty$.

Então $\mathbf{s}(L)$ não é Lie solúvel.

Faremos a seguinte definição: seja $E(\mathbf{s}(\Delta))$ o conjunto dos todos autovalores por ad $x$ em $\mathbf{s}(\Delta)$. Se $a \in \mathbf{s}(\Delta)$ é um autovetor de ad $x$, escrevemos $\lambda_{a}$ para seu autovalor. Assim $\operatorname{ad} x(a)=a^{\prime}=\lambda_{a} a$. 
Lema 5.1.10 Sejam $a, b \in E(\mathbf{s}(\Delta))$, então:

1. $a b \in E(\mathbf{s}(\Delta))$ e $\lambda_{a b}=\lambda_{a}+\lambda_{b}$;

2. $\{x a, x b\}=x\left(\lambda_{b}-\lambda_{a}\right) a b$.

Demonstração. A parte 1 segue da aplicação da regra de Leibnitz, isto é

$$
\begin{aligned}
\{x, a b\} & =b\{x, a\}+a\{x, b\} \\
& =\lambda_{a} a b+\lambda_{b} a b \\
& =\left(\lambda_{a}+\lambda_{b}\right) a b .
\end{aligned}
$$

Dessa maneira, $a b \in E(\mathbf{s}(\Delta))$. Além disso, $\lambda_{a b}=\lambda_{a}+\lambda_{b}$. A parte 2 segue de $\{x a, x b\}=x\left(a b^{\prime}-a^{\prime} b\right)$ e também de $a^{\prime}=\lambda_{a} a, b^{\prime}=\lambda_{b} b$.

O próximo resultado segue do Lema 5.1.10.

Corolário 5.1.11 Sejam $a, b \in E(\mathbf{s}(\Delta))$ com $\lambda_{a} \neq \lambda_{b}$. Se $a x, b x \in \delta_{n-1}(R)$, então $x a b \in \delta_{n}(R)$.

Lema 5.1.12 Se $l \geq 2^{n}-1$ e $a_{1}, a_{2}, \ldots, a_{l} \in V_{1}$, então $x a_{1} a_{2} \cdots a_{l} \in \delta_{n}(R)$.

Demonstração. Iremos usar indução sobre $n \geq 0$. Para $n=0$ é evidente. Antes de provar, iremos introdução a seguinte notação: para cada $i \in\{1,2, \ldots, l\}$, vamos colocar $\lambda_{i}=\lambda_{a_{i}}$. Efetivamente neste caso $\lambda_{i}=1$, para todo $i$. Vamos por $\lambda_{S}=\sum_{i \in S} \lambda_{i}$, para cada subconjunto $S \subseteq\{1,2, \ldots, l\}$. Além disso, vamos por $a_{S}=\prod_{i \in S} a_{i}$. É claro que $\lambda_{i} \neq 0$ para cada $i \in\{1,2, \ldots, l\}$. Agora, dado $n>0$, afirmamos que existem subconjuntos $U, V \subseteq\{1,2, \ldots, l\}$ com $U \cap V=\emptyset, U \cup V=\{1,2, \ldots, l\},|U|,|V| \geq 2^{n-1}-1$, de modo $\lambda_{U} \neq \lambda_{V}$.

Para provar esta afirmação, vamos supor o contrário. Indicando $\nu=\sum_{i=1}^{l} \lambda_{i}$, para cada $U \subseteq\{1,2, \ldots, l\}$ com $|U|=2^{n-1}-1$, temos que $\lambda_{U}=\nu / 2$. De fato, se $V=\bar{U}$ o complementar de $U$ em $\{1,2, \ldots, l\}$, então $|V| \geq 2^{n-1}-1$, de modo que $\lambda_{U}=\lambda_{V}$ e $\lambda_{U}+\lambda_{V}=\nu$, consequentemente $2 \lambda_{U}=\nu$. Analogamente, para cada $U$ com $|U|=2^{n-1}$ obtemos $\lambda_{U}=\nu / 2$ uma vez que $V=\bar{U}$ o complemento de $U$ é tal que $|V|=l-|U| \geq$ 
$2^{n}-1-2^{n-1}=2^{n-1}-1$, isto implica que $\lambda_{U}=\lambda_{V}$ e novamente $\lambda_{U}+\lambda_{V}=\nu$. Em particular,

$$
\lambda_{1}+\lambda_{2}+\cdots+\lambda_{2^{n-1}-1}=\nu / 2 \quad \text { e } \quad \lambda_{1}+\lambda_{2}+\cdots+\lambda_{2^{n-1}}=\nu / 2
$$

o qual implica que $\lambda_{2^{n-1}}=0$, mas isto contradiz o fato que $\lambda_{i}=1$ para todo $i$. Portanto, a afirmação é válida.

Assim, por indução segue que $x a_{U}, x a_{V} \in \delta_{n-1}(R)$. Como $\lambda_{U} \neq \lambda_{V}$, pelo Corolário 5.1.11, obtemos $x a_{U} a_{V}=x a_{1} a_{2} \cdots a_{l} \in \delta_{n}(R)$, como queríamos.

Corolário 5.1.13 Se $\delta_{n}(R)=0$, então $\operatorname{dim} L^{2} \leq 2^{n}-2$.

Demonstração. De fato, vamos supor o contrário. Então existem $a_{1}, a_{2}, \ldots, a_{2^{n}-1} \in L^{2}$ linearmente independentes de modo que $x a_{1} a_{2} \cdots a_{2^{n}-1} \neq 0$ em $R$ é um monômio da base. Mas isto contradiz o Lema 5.1.12.

Demonstração da Proposição 5.1.9. Para o caso $\lambda \neq 0$, se $\operatorname{dim} L^{2}=\infty$, então $\delta_{n}(R) \neq 0$ para todo $n$ pelo Corolário 5.1.13. Neste caso, $R$ não é Lie solúvel e finalmente concluímos a prova.

Agora, os dois casos considerados na Proposição 5.1.2 e Proposição 5.1.9 completam a prova do Teorema 3.4.1 no caso $p \neq 2$.

\subsection{Solubilidade da álgebra simétrica truncada no caso $p=2$}

Daremos uma resposta completa para a solubilidade forte. Como para problemas de solubilidades da álgebra simétrica truncada no caso $p=2$, mostraremos que o problema é diferente das outras características. Em particular, Proposição 5.1.2 e Proposição 5.1.9 não são válidas. Também, no caso char $K=2$, obtemos dois exemplos de álgebras simétricas truncadas que são solúveis mas não são solúveis forte. 
Lema 5.2.1 Considere uma álgebra de Lie sobre um corpo $K$ de característica $p=2$ dada por uma base e relações:

$$
L=\left\langle x, y_{i} \mid\left[x, y_{i}\right]=y_{i}, \quad i \in \mathbb{N}\right\rangle,
$$

os comutadores restantes sendo triviais. Então

1. $L^{2}=\Delta(L)=\Delta_{1}(L)=\left\langle y_{i} \mid i \in \mathbb{N}\right\rangle$, o qual é de dimensão infinita;

2. $\mathbf{s}(L)$ é solúvel de comprimento 3;

3. $\mathbf{s}(L)$ não é solúvel forte.

Demonstração. A primeira afirmação é checada diretamente.

Denote $H=\left\langle y_{i} \mid i \in \mathbb{N}\right\rangle$. Elementos da base de $\mathbf{s}(L)$ têm uma forma ou $y_{i_{1}} \cdots y_{i_{k}} \in$ $\mathbf{s}(H)$ ou $x y_{i_{1}} \cdots y_{i_{m}}$, onde $k, m \geq 0$. Agora, $\mathbf{s}(H)$ é abeliano e os comutadores restantes de tais monômios da base são como os catalogados abaixo:

$$
\begin{aligned}
\left\{x y_{i_{1}} \cdots y_{i_{m}}, y_{j_{1}} \cdots y_{j_{k}}\right\} & =k x y_{i_{1}} \cdots y_{i_{m}} y_{j_{1}} \cdots y_{j_{k}} \\
\left\{x y_{i_{1}} \cdots y_{i_{m}}, x y_{j_{1}} \cdots y_{j_{n}}\right\} & =(n-m) x y_{i_{1}} \cdots y_{i_{m}} y_{j_{1}} \cdots y_{j_{n}} .
\end{aligned}
$$

Assim, $\delta_{1}(\mathbf{s}(L))=\{\mathbf{s}(L), \mathbf{s}(L)\}$ é gerado por monômios acima contendo $x$. Considere os demais comutadores (5.2), eles são não nulos somente quando um dos números $m, n$ é par enquanto o outro é ímpar. Portanto, $\delta_{2}(\mathbf{s}(L))$ é gerado pelos monômios $x y_{i_{1}} \cdots y_{i_{m}}$ onde $m$ é ímpar. Finalmente, usando (5.2), ganhamos $\delta_{3}(\mathbf{s}(L))=0$.

Para provar a última afirmação, verificamos por indução sobre $k$ que $\widetilde{\delta}_{k}(\mathbf{s}(L))$ contém uma quantidade infinita de monômios $x y_{i_{1}} \cdots y_{i_{m}}$ para $m$ par e ímpar. Usamos (5.2) e o fato que podemos adicionalmente multiplicar os comutadores por $y_{s}$ faz a quantidade par ou ímpar resultante.

Lema 5.2.2 Considere uma álgebra de Lie sobre um corpo $K$ de característca $p=2$ dada por uma base e relações:

$$
L=\left\langle x, y_{i}, z_{i} \mid\left[x, y_{i}\right]=z_{i}, i \in \mathbb{N}\right\rangle
$$

os comutadores restantes sendo triviais. Então 
1. $\Delta(L)=\Delta_{1}(L)=\left\langle y_{i}, z_{i} \mid i \in \mathbb{N}\right\rangle$;

2. $L^{2}=\left\langle z_{i} \mid i \in \mathbb{N}\right\rangle$;

3. $\mathbf{s}(L)$ é solúvel de comprimento 3;

4. $\mathbf{s}(L)$ não é solúvel forte.

Demonstração. As duas primeiras afirmações são verificadas diretamente.

Denote $H=\left\langle y_{i}, z_{i} \mid i \in \mathbb{N}\right\rangle$. Seja $a \in \mathbf{s}(H)$, então denote $a^{\prime}=\{x, a\}$. Considere um monômio da base arbitrário $w \in \mathbf{s}(H)$, escreva $w=v_{1} \cdots v_{n}$, onde $v_{i}$ denota alguns elementos da base de $H$ acima. Então

$$
\begin{aligned}
w^{\prime \prime} & =\left(v_{1} \cdots v_{n}\right)^{\prime \prime}=\left(\sum_{i=1}^{n} v_{1} \cdots v_{i}^{\prime} \cdots v_{n}\right)^{\prime} \\
& =\sum_{i=1}^{n} v_{1} \cdots v_{i}^{\prime \prime} \cdots v_{n}+2 \sum_{1 \leq i<j \leq n} v_{1} \cdots v_{i}^{\prime} \cdots v_{j}^{\prime} \cdots v_{n}=0
\end{aligned}
$$

Como na prova do Lema 5.2.1, $\delta_{1}(\mathbf{s}(L))=\{\mathbf{s}(L), \mathbf{s}(L)\}$ é gerado pelos monômios contendo $x$. Seja $x a, x b, x c, x d \in \mathbf{s}(L)$ elementos da base contendo $x$, onde $a, b, c, d \in \mathbf{s}(H)$. Calculando

$$
\begin{aligned}
\delta_{2}(x a, x b, x c, x d)=\{\{x a, x b\},\{x c, x d\}\} & \\
= & \left\{x\left(a^{\prime} b+a b^{\prime}\right), x\left(c^{\prime} d+c d^{\prime}\right)\right\} \\
= & x\left(a^{\prime \prime} b+2 a^{\prime} b^{\prime}+a b^{\prime \prime}\right)\left(c^{\prime} d+c d^{\prime}\right)+x\left(a^{\prime} b+a b^{\prime}\right)\left(c^{\prime \prime} d+2 c^{\prime} d^{\prime}+c d^{\prime \prime}\right)=0 .
\end{aligned}
$$

Concluímos que $\delta_{3}(\mathbf{s}(L))=0$.

A seguir, usaremos a seguinte observação

$$
\left(y_{1} z_{2}+z_{1} y_{2}\right)^{\prime}=y_{1}^{\prime} z_{2}+y_{1} z_{2}^{\prime}+z_{1}^{\prime} y_{2}+z_{1} y_{2}^{\prime}=2 z_{1} z_{2}=0
$$


Para provar a última afirmação, usamos a possibilidade de multiplicar adicionalmente dentro a identidade de solubilidade forte

$$
\begin{aligned}
& \left\{x y_{1}, x y_{2}\right\}=x\left(y_{1} z_{2}+z_{1} y_{2}\right) ; \\
& \left\{\left\{x y_{1}, x y_{2}\right\} y_{5},\left\{x y_{3}, x y_{4}\right\} y_{6}\right\}=\left\{x\left(y_{1} z_{2}+z_{1} y_{2}\right) y_{5}, x\left(y_{3} z_{4}+z_{3} y_{4}\right) y_{6}\right\} \\
& =x\left(y_{1} z_{2}+z_{1} y_{2}\right)\left(y_{3} z_{4}+z_{3} y_{4}\right)\left(y_{5} z_{6}+z_{5} y_{6}\right) .
\end{aligned}
$$

Cada vez, inserimos novas variáveis, como $y_{5}, y_{6}$ acima, para obter que $\bar{\delta}_{n}(\ldots) \not \equiv 0$ para todo $n \geq 1$ em $\mathbf{s}(L)$.

Demonstração do Teorema 3.4.1 no caso $p=2$. Resta-nos provar que a solubilidade forte implica que $L^{2}$ tem dimensão finita. Recorde que Lema 5.1.1 é válido no caso $p=2$. A seguir, nossa prova acima é reduzida ao fato que as álgebras de Lie descritas em Proposição 5.1.2 e Proposição 5.1.9 não são válidas. Esta verificação é feita em Lema 5.2.1 e Lema 5.2.2.

Lema 5.2.3 Considere a álgebra de Poisson Hamiltoniana truncada $P=\mathbf{h}_{2}(K)$ (ou a álgebra de Poisson Hamiltoniana $P=\mathbf{H}_{2}(K)$ ) sobre um corpo $K$ de característica 2. Então

1. P é solúvel de comprimento 3;

2. P não é solúvel forte.

Demonstração. Seja $P=\mathbf{h}_{2}(K)=\langle 1, X, Y, X Y\rangle_{K}$. Verifica-se que

$$
\begin{aligned}
& \delta_{1}(P)=\{P, P\}=\langle 1, X, Y\rangle_{K} ; \\
& \delta_{2}(P)=\langle 1\rangle_{K} ; \\
& \delta_{3}(P)=0 .
\end{aligned}
$$

Como acima, checa-se que $P$ não é solúvel forte. 


\subsection{Identidades na álgebra de Poisson simétrica}

Os seguintes resultados generalizam os resultados de Shestakov mencionados anteriormente (veja Teorema 2.5.2, [44]). Agora, demonstramos o teorema.

Teorema 5.3.1 Seja L uma álgebra de Lie sobre um corpo arbitrário $K$, e $S(L)$ sua álgebra simétrica. Então as seguintes condições são equivalentes:

1. L é abeliana;

2. $S(L)$ é Lie nilpotente forte;

3. $S(L)$ é Lie nilpotente;

4. S(L) é Lie solúvel forte;

5. $S(L)$ é Lie solúvel (char $K \neq 2$ ).

Demonstração. As implicações $1 \Rightarrow 2 \Rightarrow 3 \Rightarrow 5$ e $1 \Rightarrow 4 \Rightarrow 5$ são triviais.

A única implicação não trivial é $5 \Rightarrow 1$. Vamos supor que $L$ é não abeliano e chegar numa contradição. Pelos argumentos anteriores, temos: $\operatorname{dim} L / \Delta<\infty, \Delta=\Delta_{M}(L), \Delta$ é abeliano, $K$ é algebricamente fechado. Pegando $x \notin \Delta$, pelo Lema 5.1.1, a ação de ad $x$ sobre $\Delta$ é algébrica, considere os respectivos autovetores.

Seja $\lambda \neq 0$, fazendo $\bar{x}=\frac{x}{\lambda}$, obtemos uma subálgebra de dimensão dois:

$$
H=\langle x, y \mid[x, y]=y\rangle \text {. }
$$

Fazemos os cálculos a seguir, temos

$$
\begin{aligned}
& \delta_{1}\left(x y^{\alpha_{1}}, x y^{\alpha_{2}}\right)=\left(\alpha_{2}-\alpha_{1}\right) x y^{\alpha_{1}+\alpha_{2}}, \quad \alpha_{1}, \alpha_{2} \in \mathbb{N} ; \\
& \delta_{2}\left(x y^{\alpha_{1}}, x y^{\alpha_{2}}, x y^{\alpha_{3}}, x y^{\alpha_{4}}\right) \\
& \quad=\left(\alpha_{2}-\alpha_{1}\right)\left(\alpha_{4}-\alpha_{3}\right)\left(\alpha_{3}+\alpha_{4}-\alpha_{1}-\alpha_{1}\right) x y^{\alpha_{1}+\alpha_{2}+\alpha_{3}+\alpha_{4}}, \quad \alpha_{i} \in \mathbb{N} .
\end{aligned}
$$

Continuamos o processo. Pegando inteiros apropriados $\alpha_{1}, \ldots, \alpha_{2^{k}}$, vemos que $\delta_{k}\left(x y^{\alpha_{1}}\right.$ $\left., \ldots, x y^{\alpha_{2 k}}\right) \not \equiv 0$, para todo $k \geq 0$. Uma contradição. 
Considere o caso $\lambda=0$, como anteriormente reduzimos ao caso $(\operatorname{ad} x)^{2}=0$. Assim, obtemos uma subálgebra de dimensão três:

$$
H=\langle x, y, z \mid[x, y]=z,[x, z]=[y, z]=0\rangle .
$$

Fazendo os cálculos abaixo, temos

$$
\begin{aligned}
& \delta_{1}\left(x y^{\alpha_{1}}, x y^{\alpha_{2}}\right)=\left(\alpha_{2}-\alpha_{1}\right) x y^{\alpha_{1}+\alpha_{2}-1} z, \quad \alpha_{1}, \alpha_{2} \in \mathbb{N} ; \\
& \delta_{2}\left(x y^{\alpha_{1}}, x y^{\alpha_{2}}, x y^{\alpha_{3}}, x y^{\alpha_{4}}\right) \\
& \quad=\left(\alpha_{2}-\alpha_{1}\right)\left(\alpha_{4}-\alpha_{3}\right)\left(\alpha_{3}+\alpha_{4}-\alpha_{1}-\alpha_{2}\right) x y^{\alpha_{1}+\alpha_{2}+\alpha_{3}+\alpha_{4}-3} z^{3}, \quad \alpha_{i} \in \mathbb{N} .
\end{aligned}
$$

Pegando inteiros apropriados $\alpha_{1}, \ldots, \alpha_{2^{k}}$, vemos que $\delta_{k}\left(x y^{\alpha_{1}}, \ldots, x y^{\alpha_{2^{k}}}\right) \not \equiv 0$, para todo $k \geq 0$. Portanto, $H$ não é Lie solúvel, um contradição. 


\section{Referências Bibliográficas}

[1] Bahturin Yu. A., Identical relations in Lie algebras. VNU Science Press, Utrecht, 1987.

[2] Bahturin Yu. A., Identities in the universal envelopes of Lie algebras. J. Austral. Math. Soc. 18 (1974), 10-21.

[3] Bakhturin, Yu.A., Identities of a universal enveloping algebra for a Lie superalgebra. Mat. Sb., N. Ser. 127(169) (1985), no.3(7), 384-397.

[4] Bahturin Yu.A., Mikhalev A.A., Petrogradsky V.M., Zaicev M.V., Infinite dimensional Lie superalgebras. de Gruyter Exp. Math. 7. de Gruyter, Berlin, 1992.

[5] Bahturin Yu., Petrogradsky V., Polynomial identities in smash products. J. Lie Theory 12 (2002), no. 2, 369-395.

[6] Bapat, A.; Jordan, D., Lower central series of free algebras in symmetric tensor categories. J. Algebra 373 (2013), 299-311.

[7] Berezin, F. A., Several remarks on the associative hull of a Lie algebra. (Russian) Funkcional. Anal. i Priložen 1 (1967), no. 2, 1-14.

[8] Bergen J., Riley D., Usefi H., Lie superalgebras whose enveloping algebras satisfy a non-matrix polynomial identity. Israel J. Math. 196 (2013), no. 1, 161-173.

[9] Bhandari, A., Passi, I. B. S., Lie-nilpotency indices of group algebras. Bull. London Math. Soc. 24 (1992), no. 1, 68-70.

[10] Dixmier J., Enveloping algebras. AMS, Rhode Island, 1996. 
[11] Drensky V., Free algebras and PI-algebras. Graduate course in algebra. SpringerVerlag Singapore, Singapore, 2000.

[12] Etingof, P., Kim, J., Ma., On universal Lie nilpotent associative algebras. J. Algebra 321 (2009), no. 2, 697-703.

[13] Farkas D. R., Poisson polynomial identities. Comm. Algebra 26 (1998), no. 2, 401416.

[14] Farkas D. R., Poisson polynomial identities. II. Arch. Math. (Basel) 72 (1999), no. 4, 252-260.

[15] Giambruno A., Petrogradsky V. M., Poisson identities of enveloping algebras. Arch. Math. 87 (2006), 505-515.

[16] Giambruno A., Zaicev M., Exponential codimension growth of PI algebras: an exact estimate. Adv. Math. 142 (1999), no. 2, 221-243.

[17] Gordienko, A.S., Codimension of commutators of length 4. Russ. Math. Surv. 62 (2007), no. 1, 187-189; translation from Usp. Mat. Nauk 62, (2007). No. 1, 191-192.

[18] Grishin A. V., Pchelintsev S. V., On the centers of relatively free associative algebras with the identity of Lie nilpotency. Mat. Sb. 206 (2015), no. 11, 113-130; translation in Sb. Math. 206 (2015), no. 11-12.

[19] Gupta, N., Levin, F., On the Lie ideals of a ring. J. Algebra 81 (1983), no. 1, 225-231.

[20] Jacobson N., Lie algebras. Interscience, New York. 1962.

[21] Kochetov, M. V. Identities in the smash product of the universal enveloping superalgebra of a Lie superalgebra and a group algebra. Mat. Sb. 194 (2003), no. 1, 87-102; translation in Sb. Math. 194, no. 1-2, 89-103.

[22] Kostant, B., A Lie algebra generalization of the Amitsur-Levitzki theorem. Adv. Math. 40 (1981), 155-175.

[23] Krasilnikov, A., The additive group of a Lie nilpotent associative ring. J. Algebra 392 (2013), 10-22.

[24] Kukin, G.P. The word problem and free products of Lie algebras and associative algebras. Sb. Mat. Zh. 24 (1983), no. 2, 85-96. 
[25] Latyshev V.N., On finite generation of a T-ideal with the element $\left[x_{1}, x_{2}, x_{3}, x_{4}\right]$. Sb. Math. J. 6 (1965), 1432-1434.

[26] Latysev V.N., Two remarks on PI-algebras, Sb. Mat. Ž. 4 (1963), 1120-1121.

[27] Makar-Limanov, L., Shestakov, I., Polynomial and Poisson dependence in free Poisson algebras and free Poisson fields. J. Algebra 349 (2012), no. 1, 372-379.

[28] Makar-Limanov, L., Umirbaev, U., The Freiheitssatz for Poisson algebras. J. Algebra 328 (2011), no. 1, 495-503.

[29] Mishchenko S.P., Petrogradsky V.M., Regev A., Poisson PI algebras. Trans. Amer. Math. Soc., 359 (2007), no. 10, 4669-4694.

[30] Neumann P. M., An improved bound for BFC p-group. J. Austral. Math. Soc. 11 (1970), 19-27.

[31] Neunhöffer, M., Lie Algebras-Class notes, Semester 2, Academic year 2010.

[32] Ooms A. I., The Poisson center and polynomial, maximal Poisson commutative subalgebras, especially for nilpotent Lie algebras of dimension at most seven. J. Algebra 365 (2012), 83-113.

[33] Passi I. B. S., Passman D. S., Sehgal S. K., Lie solvable group rings. Can. J. Math. 25 (1973), no. 4, 748-757.

[34] Passman D.S., Group rings satisfying a polynomial identity. J. Algebra 20 (1972), $103-117$.

[35] Passman D.S., The algebraic structure of group rings. Krieger Publ. Co., Inc., Melbourne, Fla., 1985.

[36] Passman D.S., Enveloping algebras satisfying a polynomial identity. J. Algebra 134 (1990), no. 2, 469-490.

[37] Petrogradsky V.M., The existence of an identity in a restricted envelope. Mat. Zametki 49 (1991), no. 1, 84-93; translation in Math. Notes 49 (1991), no. 1-2, 60-66.

[38] Petrogradsky V.M., Identities in the enveloping algebras for modular Lie superalgebras. J. Algebra 145 (1992), no. 1, 1-21.

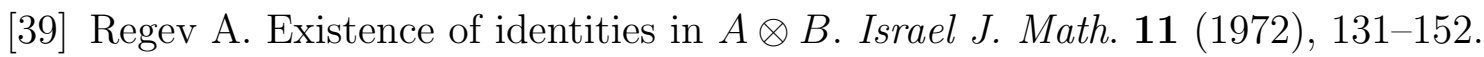


[40] Reutenauer C., Free Lie Algebras. Oxford University, New York. 1993.

[41] Riley D. M., Shalev A., Restricted Lie algebras and their envelopes. Can. J. Math. 47 (1995), 146-164.

[42] Riley D. M., Shalev A., The Lie structure of enveloping algebras, J. Algebra 162 (1993), 46-61.

[43] Sharma R. K., Srivastava J. B., Lie ideals in group rings. J. Pure Appl. Algebra 63 (1990), 63-80.

[44] Shestakov I.P., Quantization of Poisson superalgebras and speciality of Jordan Poisson superalgebras. Algebra i Logika 32 (1993), no. 5, 571-584; English translation: Algebra and Logic 32 (1993), no. 5, 309-317.

[45] Shestakov, I.; Umirbaev, U.U., The tame and the wild automorphisms of polynomial rings in three variables. J. Am. Math. Soc. 17 (2004), no. 1, 197-227.

[46] Siciliano, S., Lie derived lengths of restricted universal enveloping algebras. Publ. Math. 68 (2006), no. 3-4, 503-513.

[47] Siciliano S., Usefi H., Engel condition on enveloping algebras of Lie superalgebras. $J$. Pure Appl. Algebra 219 (2015), no. 12, 5631-5636.

[48] Siciliano S., Usefi H., Lie identities on symmetric elements of restricted enveloping algebras. Israel J. Math. 195 (2013), no. 2, 999-1012.

[49] Siciliano S., Usefi H., Lie properties of restricted enveloping algebras. Lie algebras and related topics. Contemp. Math., Amer. Math. Soc., Providence, RI 652 (2015), $141-152$.

[50] Siciliano S., Usefi H., Lie solvable enveloping algebra of characteristic two. J. Algebra 382 (2013), 314-331.

[51] Strade H., Farnsteiner R., Modular Lie algebras and their representations. Marcel Dekker, Inc., New York, 1988.

[52] Tarasov A. A., On some commutative subalgebras in the universal enveloping algebra of the Lie algebra $g l_{n}(\mathbb{C})$. Mat. Sb. 191 (2000), no. 9, 115-122; translation in $S b$. Math. 191 (2000), no. 9-10.

[53] Usefi H., Lie identities on enveloping algebras of restricted Lie superalgebras. J. Algebra 393 (2013), 120-131. 
[54] Usefi H., Non-matrix polynomial identity enveloping algebras. J. Pure Appl. Algebra 217 (2013), no. 11, 2050-2055.

[55] Vergne M., La structure de Poisson sur l'algèbre symétrique d'une algèbre de Lie nilpotente. C. R. Acad. Sc. Paris 269 (1969), Série A, 950-952.

[56] Vinberg, E. B. Some commutative subalgebras of a universal enveloping algebra. (Russian) Izv. Akad. Nauk SSSR Ser. Mat. 54 (1990), no. 1, 3-25, 221; translation in Math. USSR-Izv. 36 (1991), no. 1, 1-22.

[57] Volichenko I.B., The T-ideal generated by the element $\left[x_{1}, x_{2}, x_{3}, x_{4}\right]$. Preprint no. 22, Inst. of Mathematics of the Academy of Sciences of BSSR, Minsk 1978, 13 pp. (Russian).

[58] Zel'manov, E. I., The solution of the restricted Burnside problem for groups of odd expoents. Math. USSR Izv. 36 (1991), 41-60.

[59] Zel'manov, E. I., The solution of the restricted Burnside problem for 2-groups. Math. Sb. 182 (1991), 568-592. 